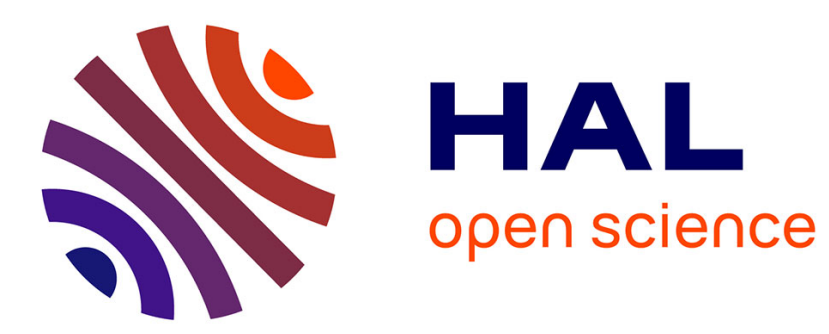

\title{
Self-Stabilizing Leader Election in Polynomial Steps
}

Karine Altisen, Alain Cournier, Stéphane Devismes, Anaïs Durand, Franck

Petit

\section{To cite this version:}

Karine Altisen, Alain Cournier, Stéphane Devismes, Anaïs Durand, Franck Petit. Self-Stabilizing Leader Election in Polynomial Steps. [Research Report] VERIMAG. 2014. hal-00980798v3

\section{HAL Id: hal-00980798 \\ https://hal.science/hal-00980798v3}

Submitted on 2 May 2014 (v3), last revised 12 Feb 2015 (v4)

HAL is a multi-disciplinary open access archive for the deposit and dissemination of scientific research documents, whether they are published or not. The documents may come from teaching and research institutions in France or abroad, or from public or private research centers.
L'archive ouverte pluridisciplinaire HAL, est destinée au dépôt et à la diffusion de documents scientifiques de niveau recherche, publiés ou non, émanant des établissements d'enseignement et de recherche français ou étrangers, des laboratoires publics ou privés. 


\title{
Self-Stabilizing Leader Election in Polynomial Steps*
}

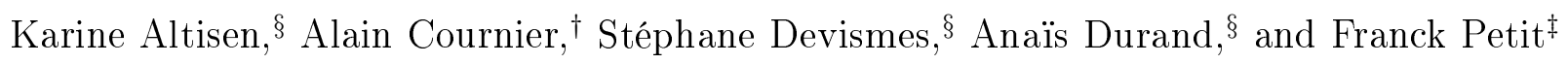 \\ $\S$ Univ. Grenoble Alpes / CNRS, VERIMAG, F-38000 Grenoble, France \\ $\dagger$ Univ. Picardie Jules Verne, MIS, Amiens, France \\ † LIP6 UMR 7606, UPMC Sorbonne Universités, Paris
}

\begin{abstract}
In this paper, we propose a silent self-stabilizing leader election algorithm for bidirectional connected identified networks of arbitrary topology. This algorithm is written in the locally shared memory model. It assumes the distributed unfair daemon, the most general scheduling hypothesis of the model. Our algorithm requires no global knowledge on the network (such as an upper bound on the diameter or the number of processes, for example).

We show that its stabilization time is in $\Theta\left(n^{3}\right)$ steps in the worst case, where $n$ is the number of processes. Its memory requirement is asymptotically optimal, i.e., $\Theta(\log n)$ bits per processes. Its round complexity is of the same order of magnitude - i.e., $\Theta(n)$ rounds - as the best existing algorithm [8] designed with similar settings (i.e., it does not use global knowledge and is proven under the unfair daemon).

To the best of our knowledge, this is the first self-stabilizing leader election algorithm for arbitrary identified networks that is proved to achieve a stabilization time polynomial in steps. By contrast, we show that the previous best existing algorithm designed with similar settings [8] may stabilize in a non polynomial number of steps.
\end{abstract}

Keywords: Distributed algorithms, fault-tolerance, self-stabilization, leader election, unfair daemon.

\section{Introduction}

In distributed computing, the leader election problem consists in distinguishing one process, so-called the leader, among the others. We consider here identified networks. So, as it is usually done, we augment the problem by requiring all processes to eventually know the identifier of the leader. The leader election is fundamental as it is a basic component to solve many other important problems, e.g., consensus, spanning tree constructions, implementing broadcasting and convergecasting methods, etc.

Self-stabilization $[9,10]$ is a versatile technique to withstand any transient fault in a distributed system: a self-stabilizing algorithm is able to recover, i.e., reach a legitimate configuration, in finite time, regardless the arbitrary initial configuration of the system, and therefore also after the occurrence of transient faults. Thus, self-stabilization makes no hypotheses on the nature or extent of transient faults that could hit the system, and recovers from the effects of those faults in a unified manner. Such versatility comes at a price. After transient faults, there is a finite period of time, called the stabilization phase, before the system returns to a legitimate configuration. The stabilization time is then the worst case duration of the stabilization phase, i.e., the maximum time to reach a legitimate configuration starting from an arbitrary one. Notice that efficiency of self-stabilizing algorithms is mainly evaluated according to their stabilization time and memory requirement.

We consider the (deterministic) ${ }^{1}$ asynchronous silent self-stabilizing leader election problem in bidirectional, connected, and identified networks of arbitrary topology. We investigate solutions to this problem which are written in the locally shared memory model introduced by Dijkstra [9]. In this model,

\footnotetext{
${ }^{*}$ This work has been partially supported by the LabEx PERSYVAL-Lab (ANR-11-LABX-0025-01) funded by the French program Investissement d'avenir.

${ }^{1}$ We only consider here deterministic algorithms.
} 
the (distributed) unfair daemon is known as the weakest scheduling assumption. Now, proving the selfstabilization of a given algorithm under such an assumption implies that the stabilization time is finite in terms of (atomic) steps. However, despite some solutions assuming all these settings (in particular the unfairness assumption) are available in the literature $[6,7,8]$, none of them is proven to achieve a polynomial upper bound in steps on its stabilization time. Rather, the time complexities of all these solutions are analyzed in terms of rounds only.

Related Work. In [11], Dolev et al showed that the silent self-stabilizing leader election requires $\Omega(\log n)$ bits per process, where $n$ is the number of processes. Self-stabilizing leader election algorithms for arbitrary connected identified networks have been proposed in the message-passing model [1, 3, 4]. First, the algorithm of Afek and Bremler [1] stabilizes in $O(n)$ rounds using $\Theta(\log n)$ bits per process. But, it assumes that the link-capacity is bounded by a value $B$, known by all processes. Two solutions that stabilize in $O(\mathcal{D})$ rounds, where $\mathcal{D}$ is the diameter of the network, have been proposed in [3,4]. However, both solutions assume that processes know some upper bound $D$ on the diameter $\mathcal{D}$; and have a memory requirement in $\Theta(\log D \log n)$ bits.

Several solutions are also given in the shared memory model [12, 2, 6, 7, 8, 13]. The algorithm proposed by Dolev and Herman [12] is not silent, works under a fair daemon, and assume that all processes know a bound $N$ on the number of processes. This solution stabilizes in $O(\mathcal{D})$ rounds using $\Theta(N \log N)$ bits per process. The algorithm of Arora and Gouda [2] works under a weakly fair daemon and assume the knowledge of some bound $N$ on the number of processes. This solution stabilizes in $O(N)$ rounds using $\Theta(\log N)$ bits per process.

Datta et al [6] propose the first self-stabilizing leader election algorithm (for arbitrary connected identified networks) proven under the (distributed) unfair daemon. This algorithm stabilizes in $O(n)$ rounds. However, the space complexity of this algorithm is unbounded. (More precisely, the algorithm requires each process to maintain an unbounded integer in its local memory.)

Solutions in $[7,8,13]$ have a memory requirement which is asymptotically optimal (i.e. in $\Theta(\log n))$. The algorithm proposed by Kravchik and Kutten [13] assumes a synchronous daemon and the stabilization time of this latter is in $O(\mathcal{D})$ rounds. The two solutions proposed by Datta et al in $[7,8]$ assume a distributed unfair daemon and have a stabilization time in $O(n)$ rounds. However, despite these two algorithms stabilize within a finite number of step (indeed, they are proved assuming an unfair daemon), no step complexity analysis is proposed. Finally, note that the algorithm proposed in [7] assumes that each process has a bit of memory which cannot be arbitrarily corrupted.

Contribution. We propose a silent self-stabilizing leader election algorithm for arbitrary connected and identified networks. Our solution is written in the locally shared memory model assuming a distributed unfair daemon, the weakest scheduling assumption. Our algorithm assumes no knowledge of any global parameter (e.g., an upper bound on $\mathcal{D}$ or $n$ ) of network. Like previous solutions of the literature $[7,8]$, it is asymptotically optimal in space (i.e., it works using $\Theta(\log n)$ bits per process), and it stabilizes in $\Theta(n)$ rounds in the worst case. Yet, contrary to those solutions, we show that our algorithm has a stabilization time in $\Theta\left(n^{3}\right)$ steps in the worst case.

For fair comparison, we have also studied the step complexity of the algorithm, noted here $\mathcal{D} \mathcal{L} \mathcal{V}$, given in [8]. This latter is the closest to ours in terms of performance. We show that its stabilization time is not polynomial, i.e., there is no constant $\alpha$ such that the stabilization time of $\mathcal{D} \mathcal{L} \mathcal{V}$ is in $O\left(n^{\alpha}\right)$ steps. More precisely, we show that fixing $\alpha$ to any constant greater than or equal to 3 , for every $\beta \geq 2$, there exists a network of $n=2^{\alpha-3} \times 8 \times \beta$ processes in which there exists a possible execution that stabilizes in $\Omega\left(n^{\alpha+1}\right)$ steps.

Roadmap. The next section is dedicated to computational model and basic definitions. In Section 3, we propose our self-stabilizing leader election algorithm. We prove its correctness in Section 4 . In the same section, we also study its stabilization time in both steps and rounds. We show that the stabilization time of the self-stabilizing leader election algorithm given in [8] is not polynomial in steps in Section 5 . We conclude in Section 6. 


\section{Computational model}

\subsection{Distributed systems}

We consider distributed systems made of $n$ processes. Each process can communicate with a subset of other processes, called its neighbors. We denote by $\mathcal{N}_{p}$ the set of neighbors of process $p$. Communications are assumed to be bidirectional, i.e. $q \in \mathcal{N}_{p}$ if and only if $p \in \mathcal{N}_{q}$. Hence, the topology of the system can be represented as a simple undirected connected graph $G=(V, E)$, where $V$ is the set of processes and $E$ is a set of edges representing (direct) communication relations. We assume that each process has a unique ID, a natural integer. IDs are stored using a constant number of bits, $b$. As commonly done in the literature, we assume that $b=\Theta(\log n)$. Moreover, by an abuse of notation, we identify a process with its ID, whenever convenient. We will also denote by $\ell$ the process of minimum ID. (So, the minimum ID will be also noted $\ell$.)

\subsection{Locally shared memory model}

We consider the locally shared memory model, in which the processes communicate using a finite number of locally shared registers, called variables. Each process can read its own variables and those of its neighbors, but can write only to its own variables. The state of a process is the vector of the values of all its variables. A configuration $\gamma$ of the system is the vector of the states of all processes. We denote by $\gamma(p)$ the state of the process $p$ in the configuration $\gamma$. We denote by $\mathcal{C}$ the set of all possible configurations.

A distributed algorithm consists of one program per process. The program of a process $p$ is a finite set of actions of the following form:

$$
\langle\text { label }\rangle::\langle\text { guard }\rangle \rightarrow\langle\text { statement }\rangle
$$

The labels are used to identify actions. The guard of an action in the program of process $p$ is a Boolean expression involving the variables of $p$ and its neighbors. If the guard of some action evaluates to true, then the action is said to be enabled at $p$. By extension, if at least one action is enabled at $p, p$ is said to be enabled. We denote by Enabled $(\gamma)$ the set of processes enabled in configuration $\gamma$. The statement of an action is a sequence of assignments on the variables of $p$. An action can be executed only when it is enabled. In this case, the execution of the action consists in executing its statement.

The asynchronism of the system is materialized by an adversary, called the daemon. In a configuration $\gamma$, if Enabled $(\gamma) \neq \emptyset$, then the daemon selects a non empty subset $S$ of Enabled $(\gamma)$ to perform an atomic step: $\forall p \in S, p$ atomically executes one of its actions enabled in $\gamma$, leading the system to a new configuration $\gamma^{\prime}$. We denote by $\mapsto$ the relation between configurations such that $\gamma \mapsto \gamma^{\prime}$ if and only if $\gamma^{\prime}$ can be reached from $\gamma$ in one atomic step. An execution is then a maximal sequence of configurations $\gamma_{0}, \gamma_{1}, \ldots$ such that $\gamma_{i-1} \mapsto \gamma_{i}, \forall i>0$. The term "maximal" means that the execution is either infinite, or ends at a terminal configuration $\gamma$ in which Enabled $(\gamma)$ is empty.

As we saw previously, each step from a configuration to another is driven by a daemon. In this paper, the daemon is supposed to be distributed and unfair. "Distributed" means that while the configuration is not terminal, the daemon should select at least one enabled process, maybe more. "Unfair" means that there is no fairness constraint, i.e., the daemon might never permit an enabled process to execute, unless it is the only enabled process.

\subsection{Rounds}

To measure the time complexity of an algorithm, we also use the notion of round. This latter allows to highlight the execution time according to the speed of the slowest process. If a process $p$ is enabled in a configuration $\gamma_{i}$ but not enabled in the next configuration $\gamma_{i+1}$ and does not execute any action between $\gamma_{i}$ and $\gamma_{i+1}$, we said that $p$ is neutralized during the step $\gamma_{i} \mapsto \gamma_{i+1}$. Neutralization of $p$ is caused by the following situation: at least one neighbor of $p$ changes its state between $\gamma_{i}$ and $\gamma_{i+1}$, and this change makes the guards of all actions of $p$ false. The first round of an execution $e$, noted $e^{\prime}$, is the minimal prefix of $e$ in which every process that is enabled in the initial configuration either executes an action or becomes neutralized. Let $e^{\prime \prime}$ be the suffix of $e$ starting from the last configuration of $e^{\prime}$. The second round of $e$ is the first round of $e^{\prime \prime}$, and so forth. 


\subsection{Self-Stabilization}

Let $\mathcal{A}$ be a distributed algorithm. Let $\mathcal{E}$ be the set of all possible executions of $\mathcal{A}$. A specification $S P$ is a predicate over $\mathcal{E}$.

$\mathcal{A}$ is self-stabilizing for $S P$ if and only if there exists a non-empty subset of configurations $\mathcal{L} \subseteq \mathcal{C}$, called legitimate configurations, such that:

- Closure: $\forall e \in \mathcal{E}$, for each step $\gamma_{i} \mapsto \gamma_{i+1} \in e, \gamma_{i} \in \mathcal{L} \Rightarrow \gamma_{i+1} \in \mathcal{L}$.

- Convergence: $\forall e \in \mathcal{E}, \exists \gamma \in e$ such that $\gamma \in \mathcal{L}$.

- Correction: $\forall e \in \mathcal{E}$ such that $e$ starts in a legitimate configuration $\gamma \in \mathcal{L}$, e satisfies $S P$.

The stabilization time is the maximum time (in steps or rounds) to reach a legitimate configuration starting from any configuration.

\subsection{Self-Stabilizing Leader Election}

We define $S P_{L E}(e)$ the specification of the leader election problem. Let Leader $: V \mapsto \mathbb{N}$ be a function defined on the state of any process $p \in V$ in the current configuration that returns the ID of the leader appointed by $p . S P_{L E}(e)$ is true if and only if:

1. For all configuration $\gamma \in e, \forall p, q \in V, \operatorname{Leader}(p)=\operatorname{Leader}(q)$ and Leader $(p)$ is the ID of some process in $V$.

2. For all step $\gamma_{i} \mapsto \gamma_{i+1} \in e, \forall p \in V$, Leader $(p)$ has the same value in $\gamma_{i}$ and $\gamma_{i+1}$.

$\mathcal{A}$ is silent if and only if every execution is finite [11]. Let $\gamma$ be a terminal configuration. The set of all possible executions starting from $\gamma$ is the singleton $\{\gamma\}$. So, if $\mathcal{A}$ is self-stabilizing and silent, $\gamma$ must be legitimate. Thus, to prove that a leader election algorithm is both self-stabilizing and silent, it is necessary and sufficient to show that:

- In every terminal configuration $\gamma, \forall p, q \in V, \operatorname{Leader}(p)=\operatorname{Leader}(q)$ and Leader $(p)$ is the ID of some process.

- Every execution is finite.

\section{Algorithm $\mathcal{L E}$}

In this section, we present a silent and self-stabilizing leader election algorithm, called $\mathcal{L} \mathcal{E}$. Its formal code is given in Algorithm 1. Starting from an arbitrary configuration, $\mathcal{L E}$ converges to a terminal configuration, where the process of minimum ID, $\ell$, is elected. More precisely, in the terminal configuration, every process $p$ knows the identifier of $\ell$ thanks to its local variable $p . i d R$; moreover a spanning tree rooted at $\ell$ is defined using two variables per process: par and level. First, $\ell$.par $=\ell$ and $\ell . l e v e l=0$. Then, for every process $p \neq \ell, p$.par points to the parent of $p$ in the tree and $p . l e v e l$ is the height of $p$ in the tree.

We present a simple algorithm for the leader election problem in Subsection 3.1. We show why this algorithm is not self-stabilizing in Subsection 3.2. Then, we explain in Subsection 3.3 how to modify this simple algorithm to make it self-stabilizing.

\subsection{Non Self-Stabilizing Leader Election}

We first consider a simplified version of $\mathcal{L} \mathcal{E}$. Starting from a predefined initial configuration, it elects $\ell$ in all $i d R$ variables and builds a spanning tree rooted at $\ell$.

Initially, every process $p$ declares itself as leader: $p . i d R=p, p \cdot p a r=p$, and $p . l e v e l=0$. So, $p$ satisfies the two following predicates:

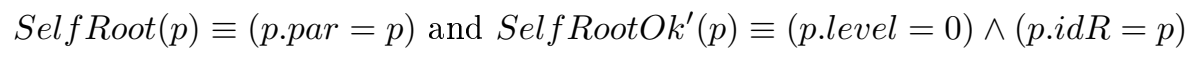

Note that, in the sequel, we say that $p$ is a self root when $\operatorname{Self} \operatorname{Root}(p)$ holds. 


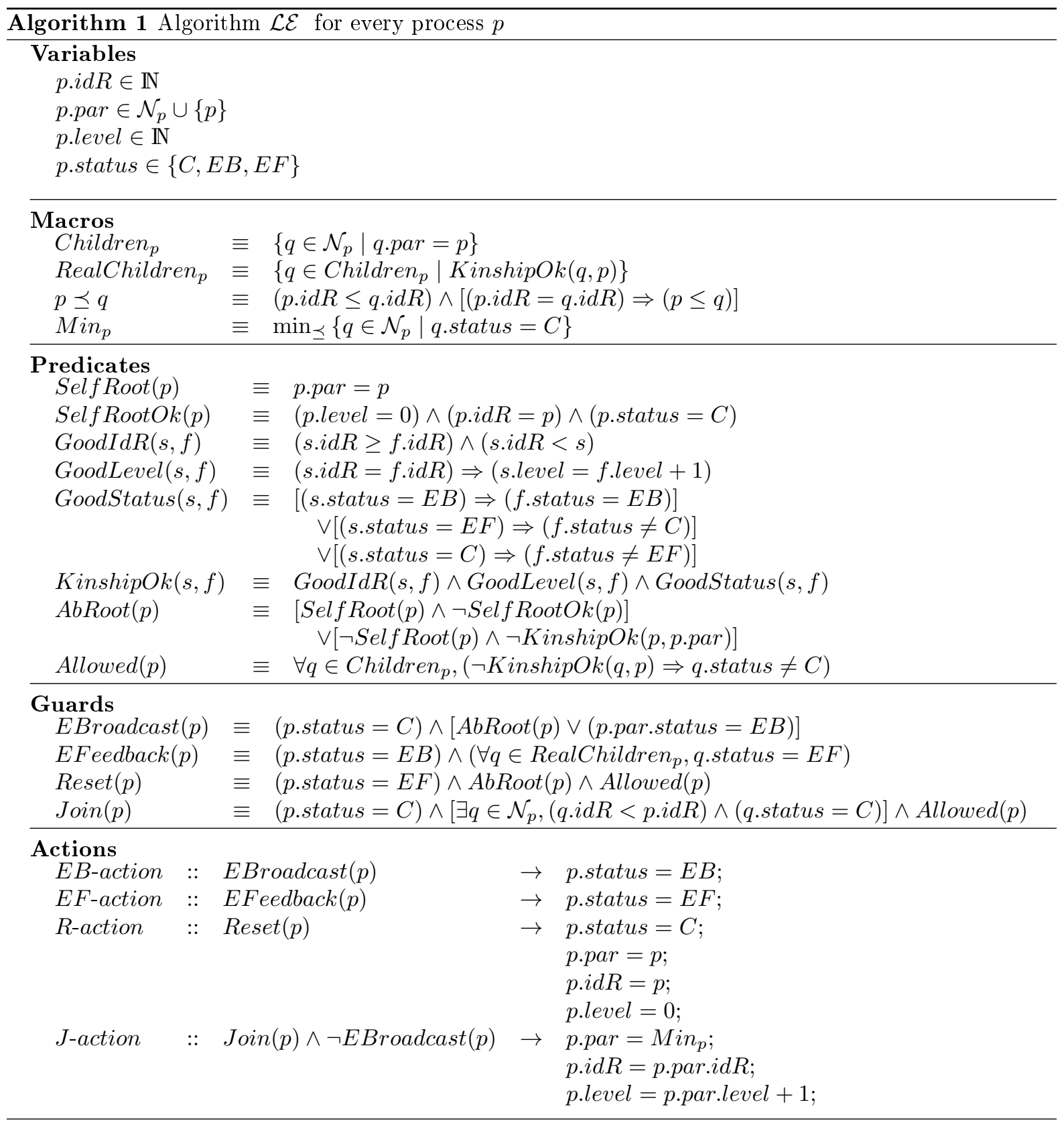


From such an initial configuration, our non self-stabilizing algorithm consists in the following single action:

$$
\begin{aligned}
& J \text {-Action } \quad:: \quad \exists q \in \mathcal{N}_{p},(q . i d R<p . i d R) \rightarrow \text { p.par }=\min _{\preceq}\left\{q \in \mathcal{N}_{p}\right\} ; \\
& \text { p.idR=p.par.idR; } \\
& \text { p.level }=\text { p.par.level }+1 ; \\
& \text { where } \forall x, y \in V, x \preceq y \Leftrightarrow(x . i d R \leq y . i d R) \wedge[(x . i d R=y . i d R) \Rightarrow(x<y)]
\end{aligned}
$$

Informally, when $p$ discovers that $p . i d R$ is not equal to the minimum identifier, it updates its variables accordingly: let $q$ be the neighbor of $p$ having $i d R$ minimal. Then, $p$ selects $q$ as new parent ( $p$.par $=q$ and $p . l e v e l=p$.par.level +1 ) and sets $p . i d R$ to the value of $q . i d R$. If there are several neighbors having $i d R$ minimal, we break ties using the identifiers of those neighbors.

Hence, the identifier of $\ell$ is propagated, from neighbors to neighbors, into the $i d R$ variables and the system reaches a terminal configuration in $O(\mathcal{D})$ rounds. Figure 1 shows an example of such an execution.

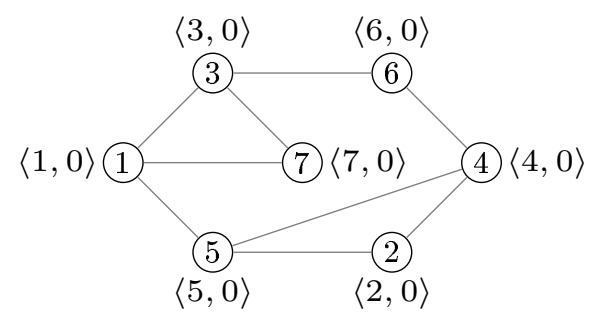

(a) Initial configuration. SelfRoot $(p) \wedge$ SelfRootOk' $(p)$ holds for every process $p$.

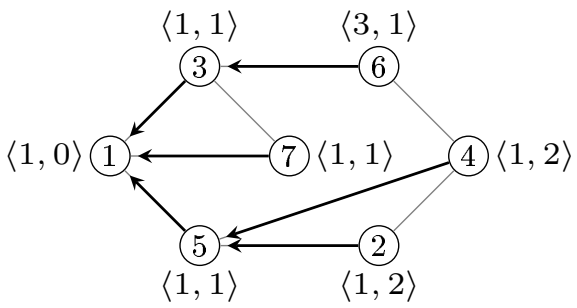

(c) 2, 3, and 4 have executed J-Action'. 3 joins the tree rooted at 1 . However, the new value of $3 . i d R$ is not yet propagated to its child 6 .

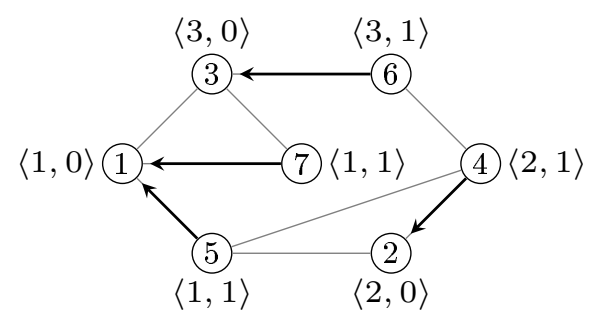

(b) 4, 5, 6, and 7 have executed J-Action'. Note that $J$-Action' was not enabled at 2 because it is a local minimum.

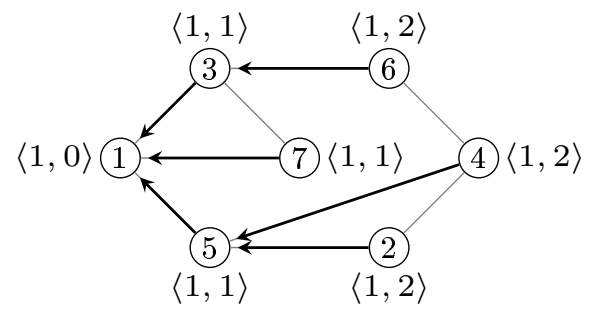

(d) 6 has executed J-Action'. The configuration is now terminal, $\ell=1$ is elected, and a tree rooted at $\ell$ is available.

Figure 1: Example of execution of the non self-stabilizing algorithm. Process identifiers are given inside the nodes. $\langle x, y\rangle$ means $i d R=x$ and level $=y$. Arrows represent par pointers. The absence of arrow means that the process is a self root.

Notice first that for every process $p, p . i d R$ is always less than or equal to its own identifier. Indeed, $p . i d R$ is initialized to $p$ and decreases each time $p$ executes $J$-Action'. Hence, $p . i d R=p$ while $p$ is a self root and after $p$ executes $J$-Action' for the first time, p.idR is smaller than its ID forever.

Second, even in this simplified context, for each two neighbors $p$ and $q$ such that $q$ is the parent of $p$, it may happens that $p . i d R$ is greater than $q . i d R$-an example is shown in Figure 1c, where $p=6$ and $q=3$. This is due to the fact that $p$ joins the tree of $q$ but meanwhile $q$ joins another tree and this change is not yet propagated to $p$. Similarly, when $p . i d R \neq q . i d R$, p.level may be different from $q$. level +1 . According to those remarks, we can deduce that when $p$.par $=q$ with $q \neq p$, we have the following relation between $p$ and $q$ :

$$
\begin{aligned}
& \operatorname{GoodIdR}(p, q) \equiv(p \cdot i d R \geq q . i d R) \wedge(p \cdot i d R<p) \\
& \operatorname{GoodLevel}(p, q) \equiv(p . i d R=q . i d R) \Rightarrow(p . \text { level }=q . \text { level }+1)
\end{aligned}
$$




\subsection{Fake IDs}

This previous algorithm is not self-stabilizing. Indeed, in a self-stabilization context, the execution may start in an arbitrary configuration. In particular, $i d R$ variables can be initialized to arbitrary natural integer values, even values that are actually not IDs of (existing) processes. We call such values fake IDs.

The existence of fake IDs may lead the system to an illegitimate terminal configuration. Refer to the example of execution given in Figure 2: starting from Configuration 2a, if processes 3 and 4 move, the system reaches the terminal configuration given in $2 \mathrm{~b}$, where there are two trees and the $i d R$ variables elect the fake ID 1.

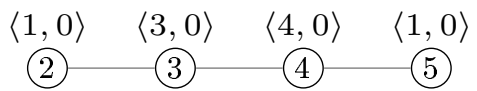

(a) Illegitimate initial configuration, where 2 and 5 have fake $i d R$.

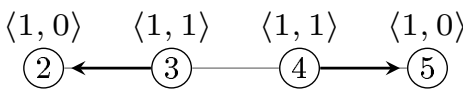

(b) 3 and 4 executed $J$-Action'. The configuration is now terminal.

Figure 2: Example of execution that does not converge to a legitimate configuration.

In this example, 2 and 5 can detect the problem. Indeed, predicate SelfRootOk' is violated by both 2 and 5 . One may believe that it is sufficient to reset the local state of 2 and 5 using the following action:

$$
R \text {-Action } \quad:: \quad \operatorname{SelfRoot}(p) \wedge \neg \operatorname{SelfRootOk}(p) \rightarrow \text { p.par }=p ; p . i d R=p ; \text { p.level }=0 ;
$$

Unfortunately, this may lead to an execution that never converges, as shown in Figure 3. Indeed, if a process resets, it becomes a self root but this does not erase the fake ID in the rest of its subtree. Then, another process can join the tree and adopt the fake ID which will be further propagated, and so on. In the example, a process resets while another joins its tree at lower level, and this leads to endless erroneous behavior, since we do not want to assume any maximal value for level (such an assumption would otherwise imply the knowledge of some upper bound on $n$ ). Therefore, the whole tree must be reset, instead of its root only. To that goal, we first froze the "abnormal" tree in order to forbid any process to join it, then the tree is reset top-down. The cleaning mechanism is detailed in the next subsection.

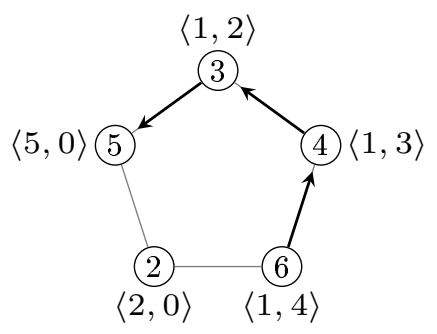

(a) Illegitimate initial configuration.

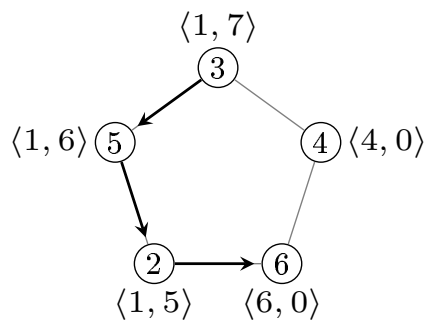

(d) Both 3 and 6 move.

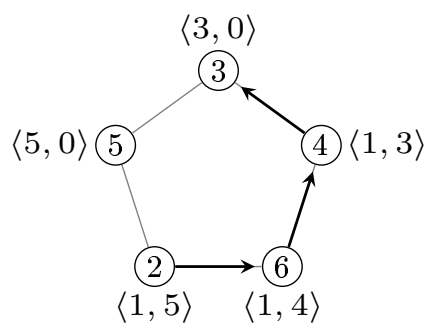

(b) 2 joins the tree. 3 leaves it.

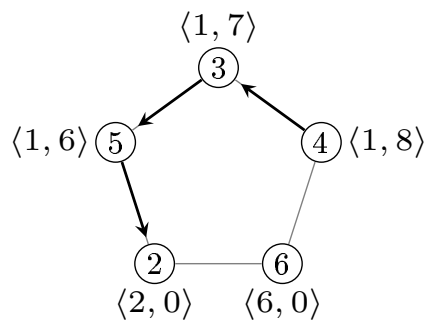

(e) 4 joins, 2 leaves.

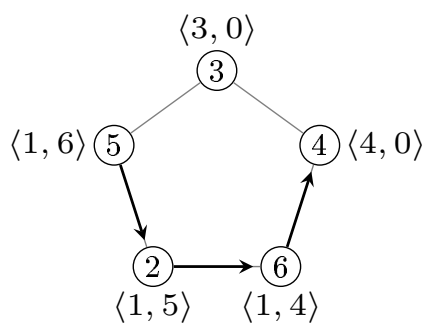

(c) 5 joins the tree. 4 leaves it.

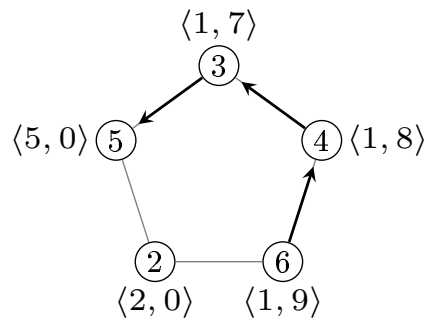

(f) Configuration similar to $3 \mathrm{a}$

Figure 3: The first process of the chain of bold arrows violates the predicate SelfRootOk' and resets by executing $R$-Action', while another process joins its tree. This cycle of resets and joins might never terminate. 


\subsection{Cleaning Abnormal Trees}

To detect possible errors (on $i d R$, par, or level), we define what is a "good relation" between a parent and its children. Namely, the predicate $\operatorname{KinshipOk}^{\prime}(p, q)$ models that a process $p$ is a real child of its parent $q=$ p.par. This predicate holds if and only if $\operatorname{GoodLevel}(p, q)$ and $\operatorname{GoodIdR}(p, q)$ are true. This relation defines a spanning forest: a tree is a maximal set of processes connected by par pointers and satisfying KinshipOk' relation. A process $p$ is a root of such a tree whenever $\operatorname{Self} \operatorname{Root}(p)$ holds or

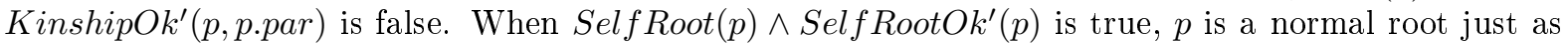
in the non self-stabilizing case (see 3.1). In other cases, there is an error and $p$ is said to be an abnormal root:

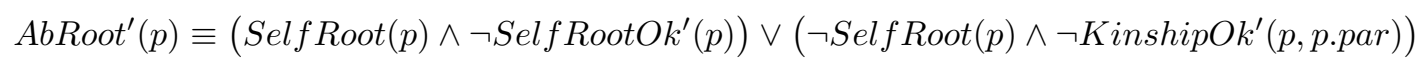

A tree is called an abnormal tree when its root is abnormal.

We now detail the different predicates and actions of Algorithm 1.

Variable status. Abnormal trees need to be frozen before to be cleaned in order to prevent them from growing endlessly (see 3.2). This mechanism is achieved using an additional variable, status, that is used as follows. If a process is clean (i.e., not involved into any freezing operation), then its status is $C$. Otherwise, it has status $E B$ or $E F$ and no neighbor can select it as its parent. These two latter states are actually used to perform a "Propagation of Information with Feedback" [5, 14] into the abnormal trees. Therefore, status EB means "Error Broadcast" and EF means "Error Feedback". From an abnormal root, the status $E B$ is broadcast down in the tree. Then, once the $E B$ wave reaches a leaf, the leaf initiates a convergecast $E F$-wave. Once the $E F$-wave reaches the abnormal root, the tree is considered to be dead, meaning that there is no process of status $C$ in the tree and no other process can join it. So, the tree can be safely reset from the abnormal root toward the leaves.

Notice that the new variable status may also get arbitrary initialization. Thus, we enforce previously introduced predicates as follows.

A self root must have status $C$, otherwise it is an abnormal root:

$$
\text { SelfRootOk }(p) \equiv \operatorname{SelfRootOk}(p) \wedge(\text { p.status }=C)
$$

To be a real child of $q, p$ should have a status coherent with the one of $q$. This is expressed with the predicate $\operatorname{GoodStatus}(p, q)$, which is used to enforce the $\operatorname{KinshipOk}(p, q)$ relation:

$$
\begin{aligned}
& \operatorname{GoodStatus}(p, q) \equiv[(\text { p.status }=E B) \Rightarrow(\text { q.status }=E B)] \vee[(\text { p.status }=E F) \Rightarrow(q . \text { status } \neq C)] \vee \\
& {[(\text { p.status }=C) \Rightarrow(\text { q.status } \neq E F)]} \\
& \operatorname{KinshipOk}(p, q) \equiv \operatorname{KinshipOk}^{\prime}(p, q) \wedge \operatorname{GoodStatus}(p, q)
\end{aligned}
$$

Precisely, when $p$ has status $C$, its parent must have status $C$ or $E B$ (if the $E B$-wave is not propagated yet to $p$ ). If $p$ has status $E B$, its parent must be of status $E B$ because $p$ gets status $E B$ from its parent and its parent will change its status to $E F$ only after $p$ gets status $E F$. Finally, if $p$ has status $E F$, its parent can have status $E B$ (if the $E F$-wave is not propagated yet to its parent) or $E F$.

Normal Execution. Remark that, after all abnormal trees have been removed, all processes have status $C$ and the algorithm works as in the initial version. Notice that the guard of $J$-action has been enforced so that only processes with status $C$ and which are not abnormal root can execute it, and when executing J-action, a process can only choose a neighbor of status $C$ as parent. Moreover, remark that the cleaning of all abnormal trees does not ensure that all fake IDs have been removed. Rather, it guarantees the removal of all fake IDs smaller than $\ell$. This implies that (at least) $\ell$ is a self root at the end of the cleaning and all other processes will elect $\ell$ within the next $\mathcal{D}$ rounds.

Cleaning Abnormal Trees. Figure 4 shows how an abnormal tree is cleaned. In the first phase (see Figure 4a), the root broadcasts status $E B$ down to its (abnormal) tree: all the processes in this tree execute $E B$-action, switch to status $E B$ and are consequently informed that they are in an abnormal tree. The second phase starts when the $E B$-wave reaches a leaf. Then, a convergecast wave of status $E F$ is initiated thanks to action EF-action (see Figure $4 \mathrm{~b}$ ). The system is asynchronous, hence all 
the processes along some branch can have status $E F$ before the broadcast of the $E B$-wave is done into another branch. In this case, the parent of these two branches waits that all its children in the tree (processes in the set RealChildren) get status EF before executing EF-action (Figure 4c). When the root gets status $E F$, all processes have status $E F$ : the tree is dead. Then (third phase), the root can reset (safely) to become a self root by executing $R$-action (Figure $4 \mathrm{e}$ ). Its former real children (of status $E F$ ) become themselves abnormal roots of dead trees (Figure 4f) and reset, etc.

Finally, we used the predicate $\operatorname{Allowed}(p)$ to temporarily lock the parent of $p$ in two particular situations - illustrated in Figure 5 - where $p$ is enabled to switch its status from $C$ to $E B$. These locks impact neither the correctness nor the complexity of $\mathcal{L} \mathcal{E}$. Rather, they allow us to simplify the proofs by ensuring that, once enabled, EB-action remains continuously enabled until executed.

\section{Correctness and Complexity Analysis}

In this section, we first define some concepts which will be used in the proofs (Subsection 4.1). Then, we show in Subsection 4.2 that Algorithm $\mathcal{L} \mathcal{E}$ is self-stabilizing and silent for the leader election, assuming a distributed unfair daemon. Along the proof, we also establish a bound on its stabilization time in steps, namely $O\left(n^{3}\right)$. Finally, we study more precisely the complexity of $\mathcal{L} \mathcal{E}$ in Subsection 4.3 (in particular, we give its complexity in rounds).

\subsection{Some definitions}

First, we instantiate the function $\operatorname{Leader}(p)$ used in the specification of the leader election (Section 2.5).

Definition 1 (Leader). For each process $p$, for every configuration $\gamma$, the value $\operatorname{Leader}(p)$ in $\gamma$ is $p . i d R$.

Note that the value of Leader $(p)$ depends on the current configuration $\gamma$. Nevertheless, when it is clear from the context, we omit the mention to $\gamma$. This will be also the case for every predicates and notations used in the sequel.

We now recall some definitions and notations from graph theory. A path $\mathcal{P}$, from $p_{k}$ to $p_{0}$ is a sequence of processes $p_{k}, p_{k-1}, \ldots, p_{0}$ such that $p_{i-1} \in \mathcal{N}_{p_{i}}$, for all $i$ in $\{1, \ldots, k\}$. Nodes $p_{k}$ and $p_{0}$ are respectively called the initial and terminal extremity of $\mathcal{P}$. The length of $\mathcal{P}$, denoted by $|\mathcal{P}|$, is equal to $k$. We call cycle any path $p_{k}, p_{k-1}, \ldots, p_{0}$ such that $p_{0}=p_{k}$. The distance between two processes $p$ and $q$, denoted $\|p, q\|$, is equal to the length of the shortest path between $p$ and $q$. The diameter of the network, denoted $\mathcal{D}$, is the maximum distance between any two processes.

The rest of the paragraph is dedicated to introducing and justifying the notion of trees induced by the KinshipOk relation. We first show that the predicate KinshipOk is an acyclic relation. To that goal, we define the graph induced by the KinshipOk relation.

Definition 2 (Kinship Relation Graph). For some configuration $\gamma$, let $G_{k r}=(V, K R)$ be a directed graph such that $(p, q) \in K R \Leftrightarrow(\{p, q\} \in E) \wedge(p \cdot p a r=q) \wedge \operatorname{KinshipOk}(p, q)$. $G_{k r}$ is called the graph of kinship relations in $\gamma$.

We first show that $G_{k r}$ is a DAG (Directed Acyclic Graph). We recall, path and cycle naturally extend to directed graph, i.e., a (directed) path $\mathcal{P}$ in $G_{k r}$ is a sequence of processes $p_{k}, p_{k-1}, \ldots, p_{0}$ such that $\left(p_{i+1}, p_{i}\right) \in K R$, for all $i$ in $\{0, \ldots, k-1\}$.

Lemma 1. Let $\gamma$ be a configuration. The graph of kinship relations in $\gamma$ contains no cycle.

Proof. By definition, for all pairs of processes $p, q$ such that $\operatorname{KinshipOk}(p, q)$ holds, we have: $p . i d R \geq$ $q . i d R$ and $p . i d R=q . i d R \Rightarrow p$. level $=q$. level +1 . Hence, the processes along any path in $G_{k r}$ are ordered w.r.t. the strict lexical order on the pair $(i d R$, level). The result directly follows.

Hence $G_{k r}$ is a DAG (Directed Acyclic Graph) and even a spanning forest since the condition $p . p a r=q$ implies at most one successor per process in $K R$. Below, we define the roots and trees of this spanning forest.

Definition 3 (Root). For some configuration $\gamma$, a process $p$ satisfies $\operatorname{Root}(p)$ (and is called a root in $\gamma$ ) if and only if $\operatorname{Self} \operatorname{Root}(p) \vee \operatorname{AbRoot}(p)$, or equivalently $\operatorname{Self} \operatorname{Root}(p) \vee \neg \operatorname{KinshipOk}(p, p$.par $)$ holds in $\gamma$. 


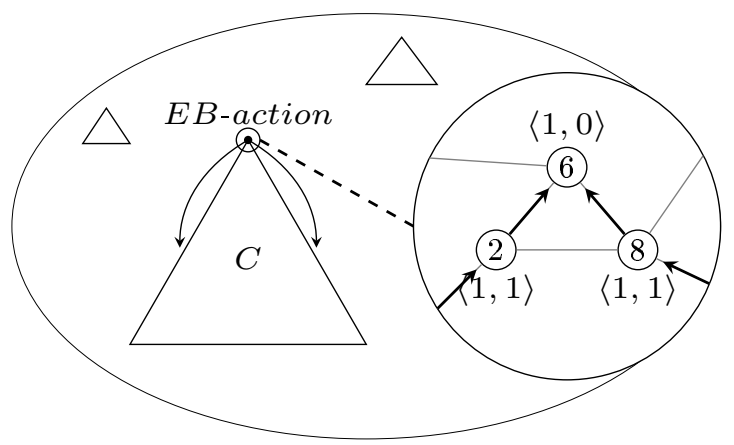

(a) When an abnormal root detects an error, it executes $E B$-action. The $E B$-wave is broadcast to the leaves. Here, 6 is an abnormal root because it is a self root and its $i d R$ is different from its ID $(1 \neq 6)$.

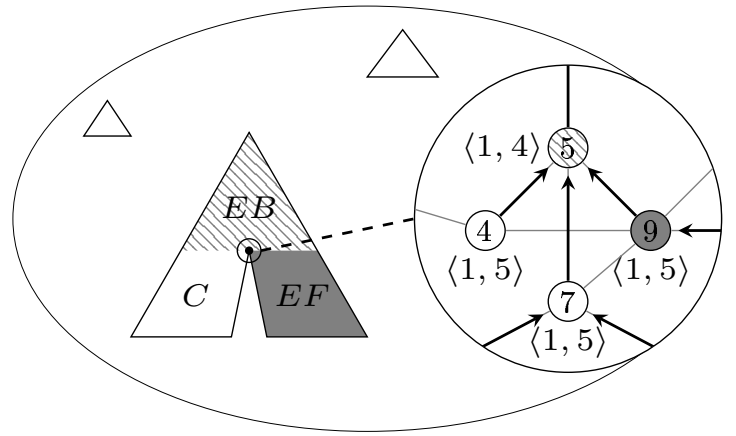

(c) It may happen that the $E F$-wave reaches a node, here process 5, even though the $E B$-wave is still broadcasting into some of its proper subtrees: 5 must wait that the status of 4 and 7 become $E F$ before executing EF-action.

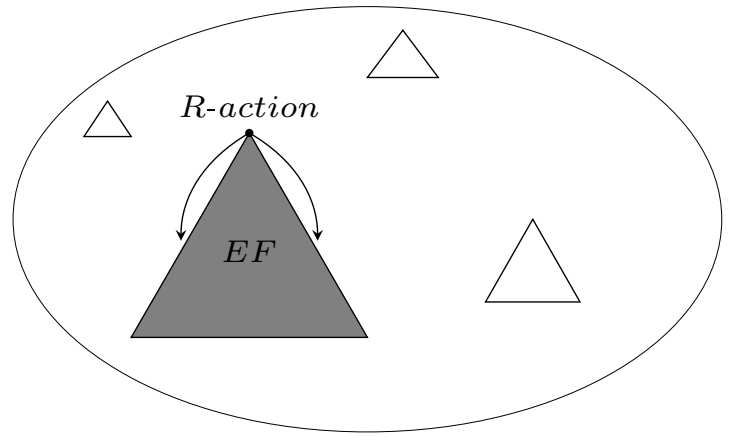

(e) $E F$-wave reaches the root. The root can safely reset (R-action) because its tree is dead. The cleaning wave is propagated down to the leaves.

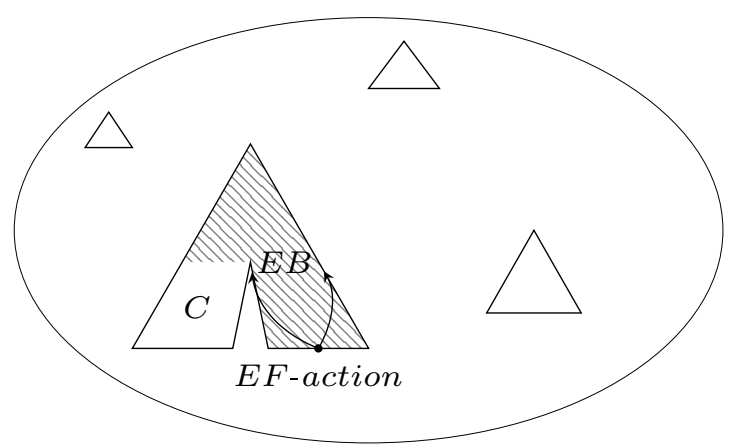

(b) When the $E B$-wave reaches a leaf, it executes $E F$-action. The EF-wave is propagated up to the root.

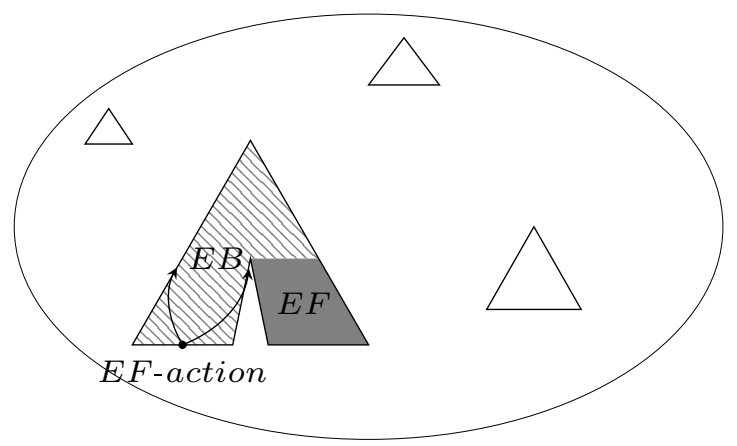

(d) $E B$-wave has been propagated in the other branch. An $E F$-wave is initiated by the leaves.

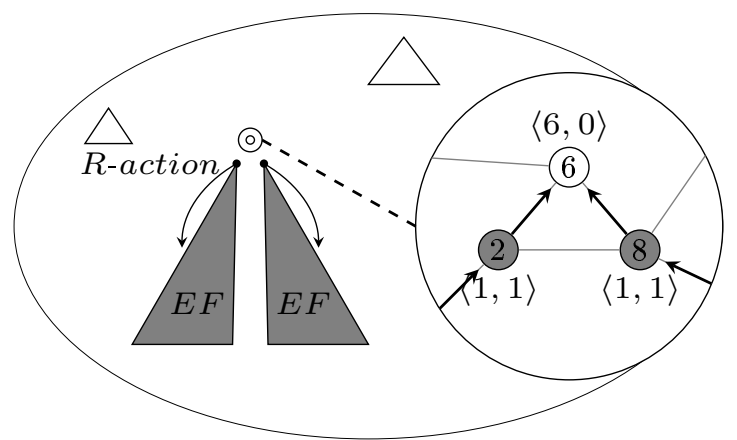

(f) Its children become themselves abnormal roots of dead trees and can execute $R$-action: 2 and 8 can clean because their status is $E F$ and their parent has status $C$.

Figure 4: Schematic example of the cleaning mechanism. Trees are filled according to the status of their processes: white for $C$, dashed for $E B$, gray for $E F$. 


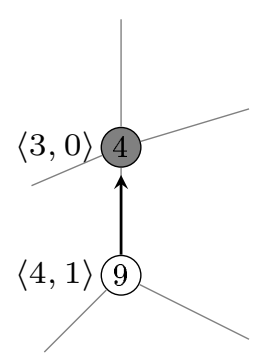

(a) 4 and 9 are abnormal roots. If 4 executes $R$-action before 9 executes EB-action, the kinship relation between 4 and 9 becomes correct and 9 is no more an abnormal root. Then, EB-action is no more enabled at 9 .

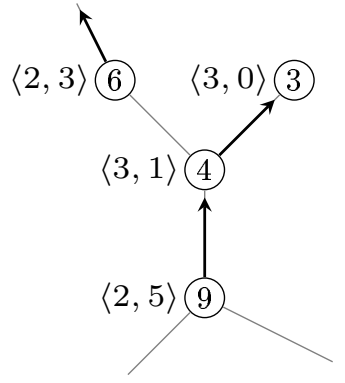

(b) 9 is an abnormal root and $\mathrm{Min}_{4}$ is 6 . If 4 executes $J$-action before 9 executes EB-action, the kinship relation between 4 and 9 becomes correct and 9 is no more an abnormal root. Then, EB-action is no more enabled at 9.

Figure 5: Example of situations where the parent of a process is locked.

Next, we define the paths, called KPaths, that follow the tree structures in $G_{k r}$, i.e., the paths linking each process to the root of its own tree.

Definition 4 (KPath). For every process $p, \operatorname{KPath}(p)$ is the unique path $p_{0}, p_{1}, \ldots, p_{k}$ such that $p_{k}=p$ and satisfying the following conditions:

- $\forall i, 1 \leq i \leq k,\left(p_{i} \cdot \operatorname{par}=p_{i-1}\right) \wedge \operatorname{KinshipOk}\left(p_{i}, p_{i-1}\right)$

- $\operatorname{Root}\left(p_{0}\right)$

Using Definitions 3 and 4, we formally define trees as follows.

Definition 5 (Tree). For some configuration $\gamma$, for every process $p$ such that $\operatorname{Root}(p)$, we define Tree $(p)$, the tree rooted at $p$, as follows:

$$
\operatorname{Tree}(p)=\{q \in V \mid p \text { is the initial extremity of } \operatorname{KPath}(q)\}
$$

This means, in particular, that we identify each tree with the ID of its root.

We give in Observation 1 an invariant on KPaths when looking at the status of the processes. This property is based on the notion of S-Trace defined below.

Definition 6 (S-Trace). For some configuration $\gamma$, for a sequence of processes $p_{0}, p_{1}, \ldots, p_{k}$, we define $S$-Trace $\left(p_{0}, p_{1}, \ldots, p_{k}\right) \in\{C, E B, E F\}^{*}$ as the sequence ( $p_{0}$. status).(p $p_{1}$. status $) \ldots$ (p $p_{k}$. status $)$ in $\gamma$.

Observation 1. For any configuration, we have: $\forall p \in V, S$-Trace $($ KPath $(p)) \in E B^{*} C^{*} \cup E B^{*} E F^{*}$.

Proof. Let $p$ be a process. If $|K \operatorname{Path}(p)|=1$, Observation 1 trivially holds. For $|K \operatorname{Path}(p)| \geq 2$, assume by contradiction that $S$-Trace $(K \operatorname{Path}(p)) \notin E B^{*} C^{*} \cup E B^{*} E F^{*}$. Then $\exists s, f \in K \operatorname{Path}(p)$ such that s.par $=f$ and $S$-Trace $(f, s) \in\{C . E B, C . E F, E F . E B, E F . C\}$. In all cases, $\neg \operatorname{GoodStatus~}(s, f)$ holds, which in turns implies that $\neg \operatorname{KinshipOk}(s, f)$. This contradicts Definition 4 .

\subsection{Correctness}

To prove the self-stabilization of Algorithm $\mathcal{L E}$ under an unfair daemon, we first show that any execution is finite (Theorem 1) and then we show that in any terminal configuration, there is a unique leader: for every two processes, $p$ and $q$, we have Leader $(p)=\operatorname{Leader}(q)$ and Leader $(p)$ is the ID of some process (Theorem 2). 


\subsubsection{Termination of $\mathcal{L E}$}

The goal, here, is to show that any execution contains a finite number of steps. We first partition a given execution into a finite number of segments (Lemma 4), see Fig. 6. Then, we prove that each segment contains a finite number of $J$-actions (Lemma 10). This latter result implies that every execution contains a finite number of $J$-actions (Corollary 2). Then, we show, in Lemma 11 and Corollary 3 , that every execution contains a finite number of other actions. This allows us to conclude in Theorem 1 that every execution contains a finite number of steps.

Abnormal Trees. First, we introduce some notions that refine the concept of trees.

Definition 7 (Normal/Abnormal Tree). For every configuration $\gamma$ and every process $p$, any tree rooted at $p$ such that $\neg A b \operatorname{Root}(p)$ in $\gamma$ is called a normal tree. In this case, $\operatorname{Self} \operatorname{Root}(p) \wedge \operatorname{Self} \operatorname{RootOk}(p)$ holds in $\gamma$, by Definition 3. Any tree that is not normal is simply said to be abnormal.

Definition 8 (Alive/Dead). Let $\gamma$ be a configuration. A process $p$ is called alive in $\gamma$ if and only if $\gamma(p)$.status $=C$. Otherwise, $p$ is said to be dead. A tree rooted at some process $r$, Tree $(r)$, in $\gamma$ is called an alive tree in $\gamma$ if and only if $\exists p \in \operatorname{Tree}(r)$ such that $p$ is alive in $\gamma$. Otherwise, it is called a dead tree.

Definition 9 (Leave/Join a Tree). Let $\gamma \mapsto \gamma^{\prime}$ be a step. If a process $p$ is in a tree $T$ in $\gamma$, but in a different tree $T^{\prime}$ in $\gamma^{\prime}$ (namely, the roots of $T$ and $T^{\prime}$ are different), we say that $p$ leaves $T$ and joins $T^{\prime}$ in $\gamma \mapsto \gamma^{\prime}$.

Remark 1. No process can join a dead tree.

Lemma 2. No alive abnormal root can be created.

Proof. Let $p$ be a process which is not an alive abnormal root in some configuration $\gamma$. This means that $p$ is dead, or $p$ is a normal root $(\operatorname{Self} \operatorname{Root}(p) \wedge \operatorname{SelfRootOk}(p)$ holds in $\gamma)$, or $p$ is not a root (KinshipOk(p,p.par) holds in $\gamma$ ).

Let $\gamma \mapsto \gamma^{\prime}$ be a step. If $p$ executes $E B$-action (respectively EF-action) during the step $\gamma \mapsto \gamma^{\prime}$ then $\gamma^{\prime}(p)$.status $=E B$ (respectively $\gamma^{\prime}(p)$.status $\left.=E F\right)$ and, consequently, $p$ is dead in $\gamma^{\prime}$.

If $p$ executes $R$-action, Self Root $(p) \wedge \operatorname{SelfRootOk}(p)$ holds in $\gamma^{\prime}$. So, $p$ is a normal root in $\gamma^{\prime}$.

If $p$ executes $J$-action, let $q=\operatorname{Min}_{p}$ in $\gamma$. By definition of $J$-action, $\gamma(q)$.status $=C, \gamma(p)$.status $=$ $\gamma^{\prime}(p)$.status $=C$ and $\gamma(p)$.idR $\leq p$ (since $p$ is not an abnormal root at $\gamma$ ). Also, $\neg$ Self Root $(p)$ holds in $\gamma^{\prime}$.

- If $q$ does not move in $\gamma \mapsto \gamma^{\prime}$, then $\gamma^{\prime}(p)$.par $=q, \gamma^{\prime}(q)$.status $=C=\gamma^{\prime}(p)$.status, $\gamma^{\prime}(p)$.level $=$ $\gamma(q)$.level $+1=\gamma^{\prime}(q)$.level $+1, \gamma^{\prime}(p) . i d R=\gamma(q) . i d R=\gamma^{\prime}(q) . i d R<\gamma(p) . i d R \leq p$. Hence, $\operatorname{KinshipOk}(p, p . p a r)$ is true in $\gamma^{\prime}$. Now, we already know that $\neg \operatorname{Self} \operatorname{Root}(p)$ holds in $\gamma^{\prime}$. Thus, $\neg \operatorname{Self} \operatorname{Root}(p) \wedge \operatorname{KinshipOk}(p, q)$ holds in $\gamma^{\prime}: p$ is not a root in $\gamma^{\prime}$, by Definition 3 .

- Assume now that $q$ moves in $\gamma \mapsto \gamma^{\prime}$. As $\gamma(q)$.status $=C, q$ can only execute EB-action or $J$-action in the step. Consequently, $\gamma^{\prime}(q) . i d R \leq \gamma(q) . i d R$.

Then, $\gamma^{\prime}(p) . i d R=\gamma(q) . i d R \geq \gamma^{\prime}(q) . i d R$ and $\gamma^{\prime}(p) . i d R=\gamma(q) . i d R<\gamma(p) . i d R \leq p$. So, $\operatorname{GoodIdR}(p, q)$ holds in $\gamma^{\prime}$.

If $q$ executes $J$-action, $\gamma^{\prime}(p) . i d R \neq \gamma^{\prime}(q)$.idR. Otherwise, $\gamma^{\prime}(p) . i d R=\gamma^{\prime}(q) . i d R$ and $\gamma^{\prime}(p)$.level $=$ $\gamma(q)$.level $+1=\gamma^{\prime}(q)$.level +1 . So GoodLevel $(p, q)$ holds in $\gamma^{\prime}$.

Finally, $\gamma^{\prime}(p)$.status $=\gamma(p)$.status $=C$ and $\gamma^{\prime}(q)$.status $\in\{C, E B\}$, so GoodStatus $(p, q)$ holds in $\gamma^{\prime}$.

Thus, $\neg \operatorname{Self} \operatorname{Root}(p) \wedge \operatorname{KinshipOk}(p, q)$ holds in $\gamma^{\prime}$ and, so, $p$ is not a root in $\gamma^{\prime}$, by Definition 3 .

Assume now that $p$ executes no action in the step $\gamma \mapsto \gamma^{\prime}$. The only way for $p$ to become an alive abnormal root is that $\gamma(p)$.par moves during the step, since the property "alive abnormal root" only depends on $p$ and p.par. Furthermore, as $p$ is not an alive abnormal root, when $p$ is a normal root in $\gamma$, it stays so, in $\gamma^{\prime}$.

Therefore, let us consider the case when $p$ is not a root in $\gamma$ and $\gamma(p)$.par moves. As $p$ changes none of its variables, the only way for it to become an alive abnormal root is to have status $C$ in $\gamma$ 
and thus in $\gamma^{\prime}$. As GoodStatus (p,p.par) holds in $\gamma$, this implies that the status of $\gamma(p)$.par is either $E B$ or $C$. Looking at case $E B, p$ is a real child of $p$.par in $\gamma$ with status $C$; hence EF-action is disabled for p.par in $\gamma$. Looking at case $C$, p.par can execute EB-action and can change only its status to $E B$ in $\gamma \mapsto \gamma^{\prime}$ : GoodStatus(p,p.par) holds in $\gamma^{\prime}$ and consequently KinshipOk $(p, p . p a r)$ holds in $\gamma^{\prime}$. p.par can also execute J-action in $\gamma \mapsto \gamma^{\prime}$. This means that in $\gamma$ and $\gamma^{\prime}$, p.par has status $C$, hence GoodStatus (p,p.par) holds in $\gamma^{\prime}$. Furthermore, p.par has a smaller value of $i d R$ in $\gamma^{\prime}$, hence $\operatorname{GoodIdR}(p, p . p a r)$ and GoodLevel(p,p.par) are satisfied in $\gamma^{\prime}$, and consequently KinshipOk(p,p.par) holds in $\gamma^{\prime}$.

Lemma 3. No alive abnormal tree can be created.

Proof. Let $\gamma \mapsto \gamma^{\prime}$ a step. Let $p \in V$. Assume there is no alive abnormal tree rooted at $p$ in $\gamma$. In particular, $p$ is not an alive abnormal root in $\gamma$. Then, assume, by contradiction, that Tree $(p)$ exists and is an alive abnormal tree in $\gamma^{\prime}$.

- If $\gamma^{\prime}(p)$.status $=E F$, then every process in the tree has status EF (Observation 1) and the tree is dead, a contradiction.

- If $\gamma^{\prime}(p)$.status $=C$, then $p$ is an alive abnormal root in $\gamma^{\prime}$. But no alive abnormal root is created (Lemma 2), a contradiction.

- If $\gamma^{\prime}(p)$.status $=E B$. Then, according to the algorithm, there are two possible cases:

$\gamma(p)$. status $=E B:$

- If $\operatorname{AbRoot}(p)$ holds in $\gamma$, then $\operatorname{Tree}(p)$ is dead in $\gamma$ (otherwise, $\operatorname{Tree}(p)$ is an abnormal alive tree in $\gamma$, a contradiction). By the definition of J-action, no process can join Tree $(p)$ in $\gamma \mapsto \gamma^{\prime}$. Moreover, as $\gamma(p)$.status $=E B$, no process $q$ in $\operatorname{Tree}(p)$ satisfies $\operatorname{Reset}(q)$ in $\gamma$, by Observation 1. Consequently, no process can leave $\operatorname{Tree}(p)$ in $\gamma \mapsto \gamma^{\prime}$. So, every process in $\operatorname{Tree}(p)$ still have status $E F$ or $E B$ in $\gamma^{\prime}$, i.e. Tree $(p)$ is still dead in $\gamma^{\prime}$, a contradiction.

- If $\neg A b \operatorname{Root}(p)$ holds in $\gamma$, then $p$ does not satisfy $\operatorname{Self} \operatorname{Root}(p)$, otherwise $\operatorname{Sel} f \operatorname{RootOk}(p)$ implies that $\gamma(p)$.status $=C$, a contradiction. So, let $q=\gamma(p)$.par $\in \mathcal{N}_{p} . \neg A b \operatorname{Root}(p)$ in $\gamma$ implies that q.status $=E B$ and $\operatorname{KinshipOk}(p, q)$ in $\gamma$. This latter also implies that $p \in$ RealChildren $_{q}$ in $\gamma$. Now, $p \in$ RealChildren $_{q}$ and p.status $=E B$ in $\gamma$ implies that $q$ is disabled in $\gamma$. Moreover, as $\gamma^{\prime}(p)$.status $=E B, p$ does not execute any action in $\gamma \mapsto \gamma^{\prime}$. So, $\neg \operatorname{AbRoot}(p)$ still holds in $\gamma^{\prime}$, a contradiction.

$\gamma(p)$.status $=C$ : Then, $\neg$ AbRoot $(p)$ holds in $\gamma$ (otherwise $p$ is an abnormal alive root in $\gamma$ ). Then, $p$ executes EB-action in $\gamma \mapsto \gamma^{\prime}$ to get status EB. So, EBroadcast $(p) \wedge \neg A b R o o t(p)$ implies that $p$.par $\neq p$ and p.par.status $=E B$ in $\gamma$. So, let $q=\gamma(p)$.par $\in \mathcal{N}_{p}$. Now p.par $\neq p \wedge$

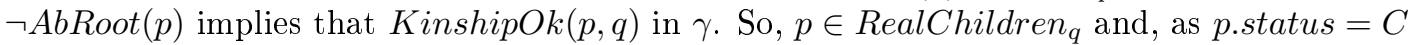
and q.status $=E B$ in $\gamma, q$ is disabled in $\gamma$. Moreover, as $\gamma^{\prime}(p)$.status $=E B, p$ necessarily executes $E B$-action in $\gamma \mapsto \gamma^{\prime}$, which only changes its status to $E B$. So, $\neg A b \operatorname{Root}(p)$ still holds in $\gamma^{\prime}$, a contradiction.

Finite Number of $J$-actions. To show that every process $p$ executes only a finite number of $J$-actions, we prove below that $p$ can only execute a finite number of $J$-actions in each segment of execution - a segment being separated from its follower by the death or the disappearance of some tree.

Definition 10 (Disappear/Die). Let $\gamma \mapsto \gamma^{\prime}$ be some step and let $p$ be a process such that $\operatorname{Root}(p)$ in $\gamma$.

$\operatorname{Tree}(p)$ disappears during the step $\gamma \mapsto \gamma^{\prime}$ if and only if $\operatorname{Tree}(p)$ is no more defined in $\gamma^{\prime}$ — namely $\operatorname{Root}(p)$ does not hold in $\gamma^{\prime}$.

$\operatorname{Tree}(p)$ dies during the step $\gamma \mapsto \gamma^{\prime}$ if and only if $\operatorname{Tree}(p)$ is alive in $\gamma$, yet $\operatorname{Tree}(p)$ exists - namely $\operatorname{Root}(p)$ holds - and is dead in $\gamma^{\prime}$. 


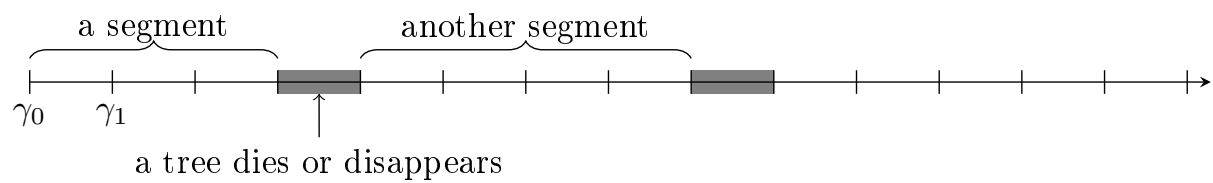

Figure 6: Segments of execution

Definition 11 (Segment of execution). Let $e=\gamma_{0} \gamma_{1} \ldots$ be any execution. $e^{\prime}=\gamma_{i} \ldots \gamma_{j}$ is a segment of execution $e$ (segment, for short) if and only if $e^{\prime}$ is a maximal factor of $e$, where no tree dies nor disappears.

Figure 6 illustrates Definition 11. We now show that the number of segments is finite.

Lemma 4. There are at most $n+1$ segments in any execution.

Proof. In the initial configuration, there are at most $n$ abnormal roots (every process) and, consequently, at most $n$ abnormal trees. As no alive abnormal tree can be created (Lemma 3 ), if an abnormal tree is alive, then it is alive since the initial configuration. So, there is at most $n$ trees that die or disappear and, consequently, there are at most $n+1$ segments in the execution.

We now count the number of $J$-actions processes can execute in a given segment. For that purpose, we first need to prove intermediate lemmas that identify properties on computation steps.

Observation 2. Let $\gamma$ be a configuration and let $p$ a process such that Reset $(p)$ is true in $\gamma$. Then Tree $(p)$ exists and is dead in $\gamma$.

Proof. Let $\gamma$ be a configuration and let $p$ be a process such that $\operatorname{Reset}(p)$ is true in $\gamma$. By definition, $\operatorname{AbRoot}(p)$ holds in $\gamma$, hence Tree $(p)$ is defined in $\gamma$. Furthermore, $\gamma(p)$.status $=$ EF : by Observation 1 , every process in $\operatorname{Tree}(p)$ has status $E F$ in $\gamma$, and we are done.

Lemma 5. Let $\gamma \mapsto \gamma^{\prime}$ be a step and let $p$ be a process such that p.status $\in\{E B, E F\}$ in $\gamma$. We note $r$ the root of the tree which contains $p$ in $\gamma$. Tree $(r)$ is an abnormal tree in $\gamma$. And if Tree $(r)$ does not disappear during the step $\gamma \mapsto \gamma^{\prime}$, p is still in Tree $(r)$ in $\gamma^{\prime}$ unless Tree $(r)$ was dead in $\gamma$.

Proof. Let $\gamma \mapsto \gamma^{\prime}$ be a step and let $p$ be a process such that p.status $\in\{E B, E F\}$ in $\gamma$. We note $r$ the root of the tree containing $p$ in $\gamma$. As $S$-Trace $(K \operatorname{Path}(p)) \in E B^{*} E F^{*}$, by Observation 1, the status of $r$ in $\gamma$ is either EF or EB. Hence $A b \operatorname{Root}(r)$ holds in $\gamma: \operatorname{Tree}(r)$ is an abnormal tree in $\gamma$.

Assume now that $\operatorname{Root}(r)$ holds in $\gamma^{\prime}$ (the tree does not disappear during the step). If $r$ executes $R$-action in $\gamma \mapsto \gamma^{\prime}$, Observation 2 applies in $\gamma$ and proves that Tree $(r)$ is dead in $\gamma$.

If $r$ does not (or cannot) execute $R$-action, its only possible action is EF-action. As Root $(r)$ holds in $\gamma^{\prime}, r$ is still abnormal root in $\gamma^{\prime}$. Let then $q \in K \operatorname{Path}(p)$ in $\gamma$ with $q \neq r$. By Observation 1 , $\gamma(q)$.status $\in\{E B, E F\}$ also. If $\gamma(q)$.status $=E B, q$ can only execute EF-action and if $\gamma(q)$.status $=$ $E F, q$ is disabled, as $q \neq r$. Executing EF-action preserves GoodStatus and hence KinshipOk relations. Therefore, the KPath from $p$ to $r$ is the same in $\gamma$ and $\gamma^{\prime}$ and then $p \in \operatorname{Tree}(r)$ in $\gamma^{\prime}$.

Lemma 6. Let $p$ be a process and let $\gamma \mapsto \gamma^{\prime}$ be step. If $p$ is an abnormal root of status $C$ in $\gamma$, then it is still an abnormal root in $\gamma^{\prime}$.

Proof. Let $\gamma \mapsto \gamma^{\prime}$ be step and let $p$ be a process such that $\operatorname{AbRoot}(p) \wedge p$.status $=C$ in $\gamma: p$ can only execute $E B$-action. Therefore, $\gamma^{\prime}(p)$.status $\in\{C, E B\}$ and every other variable of $p$ has identical value in $\gamma$ and $\gamma^{\prime}$.

So, if $\operatorname{SelfRoot}(p)$ holds in $\gamma$, then $\neg \operatorname{SelfRootOk}(p)$ in $\gamma$, and $\operatorname{SelfRoot}(p) \wedge \neg \operatorname{Self} \operatorname{RootOk}(p)$ still holds in $\gamma^{\prime}$.

Otherwise, $\neg \operatorname{SelfRoot}(p)$ holds in $\gamma$, i.e., p.par $\neq p$. Then, $\neg \operatorname{SelfRoot}(p)$ still holds in $\gamma^{\prime}$. Let $q=\gamma(p)$.par and consider the following cases:

$\gamma(q)$.status $=E F:$ Then, $\neg \operatorname{GoodStatus}(p, q)$ holds in $\gamma$, which implies $\neg$ KinshipOk $(p, q)$ holds in $\gamma$. However, $p \in$ Children $_{q}$ in $\gamma$. So, $\neg$ Allowed $(q)$ holds in $\gamma$, and $q$ is disabled. So, $\gamma^{\prime}(p)$.status $\in$ $\{C, E B\}$ and $\gamma^{\prime}(q)$.status $=E F$, which implies $\neg \operatorname{GoodStatus}(p, q)$ in $\gamma^{\prime}$. Thus, $\neg \operatorname{KinshipOk}(p, q)$ holds in $\gamma^{\prime}$. 
$\gamma(q)$. status $=E B:$ Then, $\operatorname{GoodStatus}(p, q)$ holds in $\gamma$. So, $\operatorname{AbRoot}(p)$ in $\gamma$ implies that $\neg \operatorname{GoodIdR}(p, q) \vee$ $\neg \operatorname{GoodLevel}(p, q)$ holds in $\gamma$. Now, $q$ can only executes EF-action in $\gamma \mapsto \gamma^{\prime}$. So, neither $p$ nor $q$ modify their variables par, $i d R$, or level in $\gamma \mapsto \gamma^{\prime}$, and, consequently, $\neg \operatorname{GoodIdR}(p, q) \vee$ $\neg \operatorname{GoodLevel}(p, q)$ still holds in $\gamma^{\prime}$. So, $\neg \operatorname{KinshipOk}(p, q)$ holds in $\gamma^{\prime}$.

$\gamma(q)$.status $=C: \operatorname{AbRoot}(p)$ in $\gamma$ implies that $\neg \operatorname{KinshipOk}(p, q)$ holds in $\gamma$. Thus, $\neg$ Allowed $(q)$ holds in $\gamma$ because $p \in$ Children $_{q}$ and p.status $=C$ in $\gamma$. So, $q$ cannot execute J-action in $\gamma \mapsto \gamma^{\prime}$.

Then, as $\gamma(q)$.status $=C \wedge \gamma(p)$.status $=C, \operatorname{GoodStatus}(p, q)$ holds in $\gamma$. So, AbRoot $(p)$ in $\gamma$ implies that $\neg \operatorname{GoodIdR}(p, q) \vee \neg \operatorname{GoodLevel}(p, q)$ holds in $\gamma$. As $p$ and $q$ can only modify their status in $\gamma \mapsto \gamma^{\prime}\left(q\right.$ can only execute EB-action in $\left.\gamma \mapsto \gamma^{\prime}\right), \neg \operatorname{GoodIdR}(p, q) \vee \neg \operatorname{GoodLevel}(p, q)$ still holds in $\gamma^{\prime}$. So, $\neg \operatorname{KinshipOk}(p, q)$ holds in $\gamma^{\prime}$.

In any cases, $\neg \operatorname{KinshipOk}(p, q)$ holds in $\gamma^{\prime}$. As $\neg \operatorname{SelfRoot}(p)$ holds in $\gamma^{\prime}$, AbRoot $(p)$ holds in $\gamma^{\prime}$.

Lemma 7. Let $\gamma$ be a configuration and let $p$ be a process such that p.status $\in\{E B, E F\}$ in $\gamma$. We note $r$ the root of the tree which contains $p$ in $\gamma$. Let $\gamma_{R}$ be the first configuration, if any, after $\gamma$, such that $p$ executes an $R$-action $\gamma_{R} \mapsto \gamma_{R+1}$.

Assume $\gamma_{R}$ exists, then Tree $(r)$ is dead in $\gamma_{R}$ or has disappeared (at least once) between $\gamma$ and $\gamma_{R}$.

Proof. Let $\gamma$ be a configuration and let $p$ be a process such that p.status $\in\{E B, E F\}$ in $\gamma$. We note $r$ the root of the tree which contains $p$ in $\gamma$. Let $\gamma=\gamma_{0} \gamma_{1} \ldots$ be an execution starting at $\gamma$. Let $\gamma_{R}$ be the first configuration, if any, in this execution such that $p$ executes an $R$-action during the step $\gamma_{R} \mapsto \gamma_{R+1}$.

For every configuration $\gamma_{x}, x \in\{0, \ldots, R-1\}$, the status of $p$ is $E B$ or $E F$. Hence, Lemma 5 applies iteratively in $\gamma_{x}$ : either $\operatorname{Tree}(r)$ disappears during the step $\gamma_{x} \mapsto \gamma_{x+1}$, or, if not, $p \in \operatorname{Tree}(r)$ in $\gamma_{x+1}$. Hence, in $\gamma_{R}$, either Tree $(r)$ has disappeared or, if not, $p \in \operatorname{Tree}(r)$.

When $p \in \operatorname{Tree}(r)$ in $\gamma_{R}$, by assumption, $p$ executes an $R$-action between $\gamma_{R}$ and $\gamma_{R+1}$. Hence, $\operatorname{AbRoot}(p)$ holds in $\gamma_{R}$ and thus $p=r$. Furthermore, Observation 2 applies and proves that Tree $(r)$ is dead in $\gamma_{R}$.

Lemma 8. Let $p$ be a process and let $\gamma \mapsto \gamma^{\prime}$ be a step. We note $r$ the root of the tree which contains $p$ in $\gamma$. If EBroadcast $(p)$ holds in $\gamma$, then Tree $(r)$ is an abnormal alive tree in $\gamma$ and if Tree $(r)$ has not disappeared in $\gamma^{\prime}$, p still belongs to Tree $(r)$ in $\gamma^{\prime}$.

Proof. Let $\gamma \mapsto \gamma^{\prime}$ be a step. Let $p \in V$ such that EBroadcast $(p)$ holds in $\gamma$.

If $A b \operatorname{Root}(p)$ holds in $\gamma$, then $p=r$ is the root of an alive abnormal tree, since $\gamma(p)$.status $=C$. Furthermore, if $\operatorname{Tree}(p)$ exists in $\gamma^{\prime}, p \in \operatorname{Tree}(p)$ in $\gamma^{\prime}$, trivially.

Otherwise, $\neg A b \operatorname{Root}(p)$, p.par.status $=E B$, and KinshipOk $(p, p$.par $)$ holds in $\gamma$. Applying Lemma 5 to $\gamma(p)$.par, we have that $\gamma(p)$.par belongs to an abnormal alive tree in $\gamma$ and so does $p$ : Tree $(r)$ is an alive abnormal tree.

Furthermore, first note that $\gamma(p)$.par $=\gamma^{\prime}(p)$.par ( $p$ can only change its status to $E B$ in $\gamma \mapsto \gamma^{\prime}$ : either $p$ do not move or executes EB-action). So, still by Lemma 5, in $\gamma^{\prime}$, if Tree $(r)$ exists in $\gamma^{\prime}, \gamma^{\prime}(p)$.par belongs to Tree $(r)$ in $\gamma^{\prime}$, since Tree $(r)$ is not dead in $\gamma(\gamma(p)$.status $=C)$. As KinshipOk $(p, p$.par $)$ holds in $\gamma$, we have that $p \in$ RealChildren $_{q}$ in $\gamma$. Since $\gamma(p)$.status $=C, q$ is disabled in $\gamma$ (because of $p$ ) and, as $p$ can only modify its status to $E B$ in $\gamma \mapsto \gamma^{\prime}$, we still have $p \in$ RealChildren $_{q}$ in $\gamma^{\prime}$, i.e., $p$ and $q$ belong to the same abnormal tree, Tree $(r)$, in $\gamma^{\prime}$.

Corollary 1. Let $\gamma$ be a configuration and let $p$ be a process such that EBroadcast $(p)$ holds in $\gamma$. We note $r$ the root of the tree which contains $p$ in $\gamma$. Let $\gamma_{R}$ be the first configuration, if any, since $\gamma$, such that $p$ executes an $R$-action $\gamma_{R} \mapsto \gamma_{R+1}$.

Assume $\gamma_{R}$ exists, then Tree $(r)$ is an alive abnormal tree in $\gamma$ but it is dead in $\gamma_{R}$ or has disappeared (at least once) between $\gamma$ and $\gamma_{R}$.

Proof. Let $\gamma$ be a configuration and let $p$ be a process such that $\operatorname{EBroadcast}(p)$ holds in $\gamma$. We note $r$ the root of the tree which contains $p$ in $\gamma$. Lemma 8 applies in $\gamma: \operatorname{Tree}(r)$ is an alive abnormal tree in $\gamma$.

Let $\gamma=\gamma_{0} \gamma_{1} \ldots$ be an execution starting at $\gamma$. Let $\gamma_{R}$ be the first configuration, if any, in this execution such that $p$ executes an $R$-action during the step $\gamma_{R} \mapsto \gamma_{R+1}$. We assume that $\gamma_{R}$ exists. Then at some step, $\gamma_{i} \mapsto \gamma_{i+1}, p$ executes a EB-action, with $i<R$.

Lemma 8 applies iteratively from $\gamma_{0}$ and to $\gamma_{i}$ : either Tree $(r)$ has disappeared in $\gamma_{1}$ (and so between $\gamma_{0}$ and $\gamma_{i+1}$ ), or $p$ stays in Tree $(r)$ in $\gamma_{1}$ (and so between $\gamma_{0}$ and $\gamma_{i+1}$ ), and so on. 
If $\operatorname{Tree}(r)$ has not yet disappeared in $\gamma_{i+1}, p \in \operatorname{Tree}(r)$ in $\gamma_{i+1}$ with $\gamma_{i+1}(p) . s t a t u s=$ EB. Here, Lemma 7 applies and proves that Tree $(r)$ has disappeared in $\gamma_{R}$ or $p$ is in Tree $(r)$ in $\gamma_{R}$.

Lemma 9. Let $p$ be a process. Let $s$ be a segment of execution. Between any two executions of J-action by $p$ in $s, p$ can only execute J-actions.

Proof. Let $s=\gamma_{0} \gamma_{1} \ldots$ be a segment of execution and $p \in V$. Consider two executions of $J$-action by $p$ during $s$ : one in $\gamma_{i} \mapsto \gamma_{i+1}$ and the other in $\gamma_{j} \mapsto \gamma_{j+1}$, with $i<j$. Assume by contradiction that $p$ executes an action different from $J$-action between $\gamma_{i+1}$ and $\gamma_{j}$. Let $\gamma_{k} \mapsto \gamma_{k+1}$ be the first step between $\gamma_{i+1}$ and $\gamma_{j}$ during which $p$ executes some other action: this is a EB-action. Let $\gamma_{l} \mapsto \gamma_{l+1}$ be the last step between $\gamma_{i+1}$ and $\gamma_{j}$ during which $p$ executes some other action: this is a $R$-action (hence $k<l$ ).

Now, Lemma 1 applies since in $\gamma_{k}$, EBroadcast $(p)$ holds, and in some step later $\gamma_{l} \mapsto \gamma_{l+1}, p$ executes a $R$-action. This proves that in $\gamma_{k}$, some abnormal tree is alive and that in $\gamma_{l}$, this tree is dead or has disappeared. Hence $\gamma_{k}$ and $\gamma_{l}$ are not in the same segment, a contradiction.

Lemma 10. In a segment of execution, there are at most $(n-1)(n-2) / 2$ executions of J-action.

Proof. Let $p \in V$. First, $p$ only executes J-actions between two J-actions in the same segment (Lemma 9). So, using the guard of J-action, it follows that the value of the $p . i d R$ always decreases during any sequence of $J$-actions, which means that $p$ cannot set $p . i d R$ two times to the same value during the segment.

Let $A$ be the set of processes $q$ such that q.status $=C$ at the beginning of the segment. Let $B$ the set of processes $q$ such that $q$ executes an $R$-action in the segment. $A \cap B=\emptyset$. Indeed, pick a process $q \in A \cap B . q$ switches from status $C$ at the beginning to status $E B$, and then to status $E F$ since some step later, it executes $R$-action. Hence, there exists a configuration $\gamma_{b}$ in the segment such that EBroadcast $(q)$ is true and another $\gamma_{r}$, later on such that $R$-action occurs: hence Corollary 1 applies and proves that the tree of $q$ in $\gamma_{b}$ is abnormal alive and that it dies or disappears some step before $\gamma_{r}$. This contradicts the definition of segment. Hence, $|A|+|B| \leq n$.

Now, p.idR can only get values from the $i d R$ of processes in $A$ or from the ID of processes in $B$. Let $f: V \mapsto \mathbb{N}$ such that $\forall p \in A \cup B$, if $p \in A, f(p)=x$, where $x$ is the value of $p$.idR at the beginning of the segment; otherwise, $f(p)=p$. Let $p_{0}, \ldots p_{k-1}$ (with $k \leq n$ ) be the set of processes in $A \cup B$ in ascending order of $f . p_{i}$ changes at most $i$ times of $i d R$. Hence, in a given segment, the number of executed $J$-actions, noted $\sharp J$-action, satisfies the following inequality:

$$
\sharp J \text {-action } \leq \sum_{i=0}^{k-1} i \leq \sum_{i=0}^{n-1} i=\frac{(n-1)(n-2)}{2}
$$

By Lemmas 4 and 10, in any execution, there are at most $n+1$ segments, where processes execute at most $(n-1)(n-2) / 2 J$-actions. Moreover, by definition, there are at most $n$ steps outside segments (more precisely, the steps where at least one abnormal tree dies or disappears). Hence, follows:

Corollary 2. In any execution, there are at most $\frac{n^{3}}{2}-n^{2}+\frac{n}{2}+1$ steps containing J-actions.

Other Actions. Below, we show an upper bound on the number of executions of other actions.

Lemma 11. In any execution, each process can execute at most $n R$-actions.

Proof. First, by definition, there are at most $n$ abnormal alive trees in the initial configuration. Let $\sharp A b T$ be that number. Moreover, $\sharp A b T$ can only decrease, by Lemma 3 .

Let $p$ be a process. We first show that when $p$ executes $R$-action for the first time, $\sharp A b T \leq n-1$. Then, we show that after every subsequent execution of $R$-action by $p, \sharp A b T$ necessarily decreases. Hence, we will conclude that $p$ cannot execute $R$-action more than $n$, because $\sharp A b T$ cannot be negative.

Consider the first step $\gamma_{i} \mapsto \gamma_{i+1}$ where $p$ executes $R$-action. Using Observation 2, Tree $(p)$ exists and is dead in $\gamma_{i}$. Hence, there are at most $n-1$ abnormal alive trees in $\gamma_{i}$.

Consider the $j$-th execution of $R$-action by $p$, with $j>1$. After the $(j-1)-t h R$-action of $p$, the status of $p$ is $C$. So, between the $(j-1)-t h$ and the $j-t h R$-action, the status of $p$ thus switches from 
$C$ to $E B$ and from $C$ to $E F$, so that $p$ can switch its status from $E F$ to $C$ when executing its $j-t h$ $R$-action. Hence, meanwhile there exists a configuration $\gamma_{b}$ such that EBroadcast $(q)$ is true and another $\gamma_{r}$, later on such that $p$ executes its $j-$ th $R$-action in $\gamma_{r} \mapsto \gamma_{r+1}$ : Corollary 1 applies and proves that the tree that $p$ belongs to in $\gamma_{b}$ is abnormal alive and that tree dies or disappears some step before $\gamma_{r}$, and we are done.

Let $p$ be a process. $p$ necessarily executes $R$-action between two executions of EF-action (resp. EB-action). Hence, we have the following corollary.

Corollary 3. In any execution, a process can execute EB-action and EF-action at most $n$ times, each.

By Corollaries 2, 3, and Lemma 11:

Theorem 1 (Convergence). Every execution contains at most $\frac{n^{3}}{2}+2 n^{2}+\frac{n}{2}+1$ steps.

\subsubsection{Terminal Configurations}

We now show that in a terminal configuration, there is one and only one leader process, known by all processes, i.e., for every two processes, $p$ and $q$, we have Leader $(p)=\operatorname{Leader}(q)$ and Leader $(p)$ is the ID of some process.

Lemma 12. In a terminal configuration, every process has status $C$.

Proof. By contradiction, consider a terminal configuration $\gamma$ where some process $p$ satisfies p.status $\neq C$. Then two cases are possible:

1. p.status $=E B$. By Observation $1, \exists q \in V$ such that q.status $=E B \wedge\left(\forall q^{\prime} \in\right.$ RealChildren $_{q}, q^{\prime}$. status $^{\prime} \neq$ $E B) \wedge p \in K \operatorname{Path}(q)$. If RealChildren $q=\emptyset$, then $q$ can executes $E F$-action. Otherwise, there are two cases. Either $\forall q^{\prime} \in$ RealChildren $q, q^{\prime}$. status $=E F$ and $q$ can execute EF-action, or $\exists q^{\prime} \in$ RealChildren $q, q^{\prime}$. status $=C$ then $q^{\prime}$ can execute EB-action. Hence, in both cases, $\gamma$ is not terminal, a contradiction.

2. p.status $=E F$. By Observation $1, \exists q \in V$ such that q.status $=E F \wedge(\operatorname{Root}(q) \vee($ KinshipOk $(q, q . p a r) \wedge$ q.par.status $\neq E F) \wedge q \in K \operatorname{Path}(p)$.

If $\operatorname{Root}(q)$, then $A b \operatorname{Root}(q) \vee \operatorname{SelfRoot}(q)$. Now, q.status $=E F$ implies that $A b \operatorname{Root}(q)$ holds. So, in all cases, q.status $=E F \wedge \operatorname{AbRoot}(q)$ holds. If Allowed $(q)$ holds, then $R$-action is enabled at

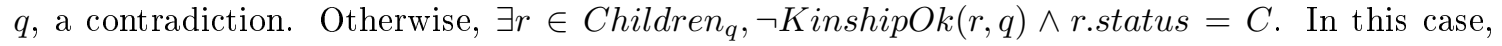
$E B$-action is enabled at $r$, a contradiction.

If $\neg \operatorname{Root}(q)$, then there are two cases. Either q.par.status $=C, \operatorname{AbRoot}(q)$ holds and we obtain a contradiction as in the case where $\operatorname{Root}(q)$ holds. Or,q.par.status $=E B$ and using the same argument as in case 1, we can deduce that some process is enabled, a contradiction.

Hence, all cases, $\gamma$ is not terminal, a contradiction.

Theorem 2 (Correctness). In a terminal configuration, $\forall p, q \in V$, Leader $(p)=\operatorname{Leader}(q)$ and Leader $(p)$ is the ID of some process.

Proof. Let $\gamma$ a terminal configuration. Assume first, by contradiction, that there are at least two leaders. Then, $G$ being connected, $\exists p, q \in V$ such that $\operatorname{Leader}(\gamma(p)) \neq \operatorname{Leader}(\gamma(q))$ and $q \in \mathcal{N}_{p}$. Assume without loss of generality that Leader $(\gamma(p))=\gamma(p) . i d R<\gamma(q)$.idR $=\operatorname{Leader}(\gamma(q))$. By Lemma 12, p.status $=$ q.status $=C$. Then, either EBroadcast $(q)$ is true and $q$ can execute EB-action or $q$ can execute $J$-action. Hence $\gamma$ is not terminal, a contradiction.

Assume now that the leader is not one of the processes, i.e., is a fake ID. Let $p \in V$ such that its level is minimum. Notice that $\gamma(p)$.status $=C$ by Lemma 12. If $\operatorname{Self} \operatorname{Root}(p)$ holds in $\gamma, \gamma(p) . i d R \neq p$. So, $\operatorname{AbRoot}(p)$ holds and $p$ can execute EB-action. Otherwise, there is $q \in \mathcal{N}_{p}$ such that $\gamma(p)$.par $=q$. The level of $p$ being minimum, we have $\gamma(p)$.level $\leq \gamma(q)$.level. So, AbRoot $(p)$ holds and $p$ can execute EB-action. Hence, $\gamma$ is not terminal, a contradiction. 
Using Theorem 2, there is exactly one root in a terminal configuration (the leader elected). So the graph of kinship relations in a terminal configuration contains exactly one tree. Hence, we can conclude:

Remark 2. In a terminal configuration, $G_{k r}$ is a spanning tree rooted at the leader.

Theorems 1 and 2 establish the self-stabilization, silence, and step complexity of Algorithm $\mathcal{L}$. Moreover, note that $i d R$ and level can be stored in $\Theta(\log n)$ bits. Hence, we can conclude:

Theorem 3. Algorithm $\mathcal{L} \mathcal{E}$ is a self-stabilizing and silent leader election algorithm working under a distributed unfair daemon. Its step complexity is at most $\frac{n^{3}}{2}+2 n^{2}+\frac{n}{2}+1$ steps. Its memory requirement is $\Theta(\log n)$ bits per process.

\subsection{Complexity Analysis}

In this section, we study the complexity of Algorithm $\mathcal{L E}$ in rounds.

\subsubsection{Stabilization Time in Rounds}

Clean Configurations. First, we study the "good" cases, i.e., when the system is in a clean configuration (defined below). From such configurations, the execution consists in building a tree rooted at $\ell$ using $J$-action only. Once, the tree is built, the system is in a terminal configuration, where every process has elected $\ell$.

Definition 12 (Clean configuration). A configuration $\gamma$ is called a clean configuration if and only if for every process $p, \neg E$ Broadcast $(p) \wedge$ p.status $=C$ holds in $\gamma$. A configuration that is not clean is said to be dirty.

Remark 3. By definition, in a clean configuration, every process $p$ has status $C$ and either $p$ is a normal root, i.e., $\operatorname{Self} \operatorname{Root}(p) \wedge \operatorname{SelfRootOk}(p)$, or (exclusively) KinshipOk(p,p.par) holds.

Remark 4. Notice that in a clean configuration, the only action a process $p$ can execute is $J$-action, provided that $\operatorname{Join}(p)$ holds. Note also that Allowed $(p)$ always holds due to Remark 3 . Verifying $\operatorname{Join}(p)$ then reduces to: $\exists q \in \mathcal{N}_{p},(q . i d R<p . i d R)$. In this case, the value of $p . i d R$ can only decrease.

Lemmas 13 to 16 proves that, starting from a clean configuration, the system reaches in $O(\mathcal{D})$ rounds a terminal configuration (see Theorem 4). We first show the set of clean configurations is closed.

Lemma 13. The set of clean configurations is closed.

Proof. Let $\gamma \mapsto \gamma^{\prime}$ be a step such that $\gamma$ is a clean configuration. By definition, all processes have status $C$ in $\gamma$. So, processes can only execute J-action (Remark 4) in $\gamma \mapsto \gamma^{\prime}$, and consequently all processes have status $C$ in $\gamma^{\prime}$. Now, $\forall p \in V, \neg E$ Broadcast $(p) \wedge p$.status $=C$ in $\gamma$ implies that there is no alive abnormal root in $\gamma$. By Lemma 2, there is no alive abnormal root in $\gamma^{\prime}$ too. Now, the fact that all processes have status $C$ and there is no alive abnormal root in $\gamma^{\prime}$ implies that $\forall p \in V, \neg E$ Broadcast $(p) \wedge$ p.status $=C$ in $\gamma^{\prime}$, i.e., $\gamma^{\prime}$ is clean.

Using Lemma 13, we show below that if a process is enabled in a clean configuration - for the only action it can execute, i.e., J-action - it remains enabled until it executes it.

Lemma 14. In a clean configuration, if J-action is enabled at $p$, it remains enabled until it is executed by $p$.

Proof. Let $\gamma \mapsto \gamma^{\prime}$ be a step such that $\gamma$ is a clean configuration. Assume by contradiction that $J$-action is enabled at $p$ in $\gamma$ and not in $\gamma^{\prime}$, but $p$ did not execute $J$-action between $\gamma$ and $\gamma^{\prime}$. By Lemma 13, $\gamma^{\prime}$ is also a clean configuration. So, $\neg$ EBroadcast $(p) \wedge$ p.status $=C$ holds in $\gamma^{\prime}$.

But $\operatorname{Join}(p)$ must be false in $\gamma^{\prime}$. Using Remark 4, this means that there necessarily exists a neighbor of $p$, say $q$, such that $\gamma(q) . i d R<\gamma(p) . i d R$ but $\gamma^{\prime}(q) . i d R \geq \gamma^{\prime}(p) . i d R=\gamma(p) . i d R$. This contradicts Remark 4.

Lemma 15. There is no (fake) idR smaller than $\ell$ in a clean configuration. 
Proof. Let $\gamma$ be a clean configuration. Assume there exists a process of $i d R$ smaller than $\ell$. Let $p$ be such a process such that p.idR is minimum among all the processes and p.level is minimum among all the processes having $i d R$ minimum.

Note that $p . i d R \neq p$ and consequently $\operatorname{SelfRootOk}(p)$ is false in $\gamma$. Hence (Remark 3), KinshipOk(p,p.par) holds in $\gamma$. Since we take $p$ of minimum $i d R, p . i d R \leq p$.par.idR in $\gamma$. As $\operatorname{GoodIdR}(p, p . p a r)$ implies that $p . i d R \geq p . p a r . i d R, p . i d R=$ p.par.idR. Now, GoodLevel $(p, p . p a r)$ implies that p.level $=$ p.par.level +1 , which contradicts the minimality of p.level.

For any process $p, p$ can only set $p . i d R$ to its own ID or copy the value of $q . i d R$, where $q$ is one of its neighbors. So, we have the following remark:

Remark 5. No fake ID is created during any step.

Lemma 16. In a clean configuration, if the idR of a process $p$ is $\ell, p$ is disable forever.

Proof. Let $\gamma$ be a clean configuration. Let $p$ be a process with $\gamma(p) . i d R=\ell$. By Remark 4 , only $J$-action can be enabled in $\gamma$ and its guard reduces to $\exists q \in \mathcal{N}_{p},(q . i d R<p . i d R)$. But Lemma 15 ensures that this cannot be true, hence $p$ is disabled in $\gamma$. Then, by Lemma 13 and Remark 5, this will be true forever.

Corollary 4. A clean configuration where $\forall p \in V, p . i d R=\ell$, is terminal.

Theorem 4. In a clean configuration, the system reaches a terminal configuration where $\forall p \in V, p . i d R=$ $\ell$ in at most $\mathcal{D}$ rounds.

Proof. Consider any execution $e$ that starts from a clean configuration. In the following, we denote by $\rho_{i}$ the first configuration of the $i$ th round in $e$. We show by induction on the distance $d \geq 0$ between the processes and $\ell$ that $\forall p \in V$ such that $\|p, \ell\| \leq d, \rho_{d}(p) . i d R=\ell$.

Base case: If $\|p, \ell\|=0, p=\ell$. Note that $\operatorname{KinshipOk}(p, p . p a r)$ cannot hold in $\rho_{0}$ since GoodIdR(p,p.par $)$ would implies that $p . i d R<p$ which is false by Lemma 15 . Hence, from Remark $3, \operatorname{Self} \operatorname{Root}(p) \wedge$ $\operatorname{SelfRootOk}(p)$ holds in $\rho_{0}$ and $\rho_{0}(p) . i d R=p=\ell$.

Induction step: Assume the property holds at some $d \geq 0$. If $\|p, \ell\|=d+1, \exists q \in \mathcal{N}_{p}$ such that $\|q, \ell\|=d$. By induction hypothesis and by Lemma 16, q.idR= $\ell$ and $q$ is disabled forever since $\rho_{d}$. If $p . i d R=\ell$ in $\rho_{d}$, it remains so forever (Lemma 16). If $p . i d R \neq \ell$ in $\rho_{d}$ then $q . i d R<$ $p . i d R$ (Lemma 15). Then, $J$-action is enabled at $p$ in $\rho_{d}$ and remains enabled until $p$ executes it (Lemma 14). As there is no fake ID smaller than $\ell$ (Lemma 15), $p . i d R=\ell$ after $p$ executes $J$-action, i.e., after at most one round. Hence, $p . i d R=\ell$ in $\rho_{d+1}$.

As $\mathcal{D} \geq \max \{\|p, \ell\|, p \in V\}$, in at most $\mathcal{D}$ rounds, the system reaches a configuration where $\forall p \in$ $V, p . i d R=\ell$. By Corollary 4, this configuration is terminal.

Dirty Configurations. In the previous section, we showed that, if the initial configuration is clean, the system reaches a terminal configuration in at most $\mathcal{D}$ rounds. But what happens if the initial configuration is dirty, i.e., if there is a process $p$ such that EBroadcast $(p)$ holds or p.status $\neq C$. In this section, we prove that starting from a dirty configuration, the system reaches a clean configuration in at most $3 n$ rounds. More precisely, we show that a dirty configuration contains abnormal trees that are "cleaned" in at most $3 n$ rounds. The system will be in a clean configuration afterwards.

Lemma 17. In an dirty configuration, there exists at least one abnormal root.

Proof. Let $\gamma$ be a dirty configuration. Then $\exists p \in V$ such that p.status $\neq C \vee E$ Broadcast $(p)$. We search for an abnormal root.

1. If p.status $\in\{E B, E F\}$, using Observation 1, there is $q \in \operatorname{KPath}(p)$ such that q.status $\in$ $\{E B, E F\} \wedge \operatorname{Root}(q)$. Then, AbRoot $(q) \vee \operatorname{SelfRoot}(q)$. Now, SelfRoot $(q) \wedge q$.status $\in\{E B, E F\}$ implies $\operatorname{AbRoot}(q)$. Hence, in all cases, $\operatorname{AbRoot}(q)$ holds.

2. If EBroadcast $(p)$ holds, Lemma 8 applies and we are done. 
We have just shown that there are abnormal roots (and so abnormal trees) in dirty configurations. Below, we prove that these abnormal trees will disappear after three waves of "cleaning". After the first wave, an abnormal tree becomes dead (Theorem 5), after the second wave any abnormal root gets the status EF (Theorem 6) and finally after the third wave there is no more abnormal trees (Theorem 7 ), hence the system is in a clean configuration.

The following technical lemma is used in the proof of Theorem 5 .

Lemma 18. When EB-action is enabled at a process $p$, it remains enabled until $p$ executes EB-action.

Proof. Assume that EB-action is enabled at a process $p$ in a configuration $\gamma$, but $p$ did not execute $E B$-action during the step $\gamma \mapsto \gamma^{\prime}$. Notice that $p$ does not execute any action during this step, as guards are mutually exclusive. As EB-action is enabled in $\gamma, \gamma(p)$.status $=C$ and then, $\gamma^{\prime}(p)$.status $=C$.

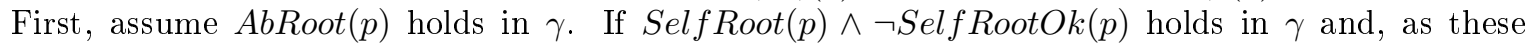
predicates only depends on the local state of $p$ and as $p$ does not execute any action during the step, it also holds in $\gamma^{\prime}$ : the action is still enabled in $\gamma^{\prime}$. Otherwise, $\neg \operatorname{Self} \operatorname{Root}(p) \wedge \neg \operatorname{KinshipOk}(p, p$.par $)$ holds in $\gamma$. These predicates only depends on the local state of $p$ and its parent. Now, Allowed(p.par $)$ does not hold in $\gamma$ because of $p$, so p.par cannot execute $R$-action nor J-action during $\gamma \mapsto \gamma^{\prime}$. Then, either p.par executes EF-action, changes its status to $E F$ and GoodStatus (p,p.par) is false in $\gamma^{\prime}$, or it executes EB-action and changes its status to $E B$. In these two cases, EBroadcast $(p)$ holds in $\gamma^{\prime}$.

Now, assume p.par.status $=E B$, p.par can only execute EF-action and change its status to EF. Then, GoodStatus (p, p.par) is false in $\gamma^{\prime}$, which implies that $\operatorname{EBroadcast}(p)$ holds in $\gamma^{\prime}$.

Theorem 5. In at most $n$ rounds, the system reaches a configuration where every abnormal tree (if any) is dead.

Proof. Consider any execution $e=\gamma_{0}, \ldots$. We denote by $\gamma_{R_{0}}$ the initial configuration of $e$. Then, $\forall i>0$, $\gamma_{R_{i}}$ both the last configuration of the $i$ th round and the first configuration of the $i+1$ th round of $e$. We show by induction on the length of the KPaths that, $\forall i \geq R_{d}(d \geq 1), \forall p \in V$, if $p$ is in an abnormal tree and $|K \operatorname{Path}(p)| \leq d$ in $\gamma_{i}$, then $p$ is dead in $\gamma_{i}$.

Base Case: If $p$ is in an abnormal tree and $|K \operatorname{Path}(p)|=1, p$ is an abnormal root. As no alive abnormal root is created (Lemma 2), if $p$ is alive, it is an alive abnormal root since $\gamma_{R_{0}}$ and if predicate $(p . s t a t u s=C \wedge \operatorname{AbRoot}(p))$ becomes false in some configuration, then it remains false forever. Hence, it is sufficient to show that any alive abnormal root is no more an alive abnormal root after one round (that is, from $\gamma_{R_{1}}$ ).

By definition, EB-action is enabled at $p$ in $\gamma_{R_{0}}$ and $p$ executes $E B$-action during the first round (Lemma 18). Hence, $p$ is dead at the end of the first round, and we are done.

Induction Hypothesis: Let $d \geq 1$. Assume that $\forall i \geq R_{d}, \forall p \in V$, if $p$ is in an abnormal tree and $|K \operatorname{Path}(p)| \leq d$ in $\gamma_{i}$, then $p$ is dead in $\gamma_{i}$.

Induction Step: We first show that for every $p \in V$, for every $i \geq R_{d}$, if (p.status $=C \wedge|K \operatorname{Path}(p)| \leq$ $d+1)$ is false in configuration $\gamma_{i}$, then for every $j \geq i,($ pstatus $=C \wedge|K \operatorname{Path}(p)| \leq d+1)$ is false in configuration $\gamma_{j}$.

Assume by contradiction that the predicate "p.status $=C \wedge|K \operatorname{Path}(p)| \leq d+1$ " is false in $\gamma_{j}$, but true in $\gamma_{j+1}(j \geq i)$. By induction hypothesis, $|K \operatorname{Path}(p)|=d+1>1$ in $\gamma_{j+1}$ (indeed, $p$ is alive in $\left.\gamma_{j+1}\right)$. So, $\gamma_{j+1}(p)$.par $\neq p$. So, let $q \in \mathcal{N}_{p}$ such that $\gamma_{j+1}(p)$.par $=q$. By definition, $|K \operatorname{Path}(q)|=d$ in $\gamma_{j+1}$. By induction hypothesis, $\gamma_{j+1}(q)$.status $\in\{E B, E F\}$. Now, p.status $=C$ and $|K \operatorname{Path}(p)|>1$ in $\gamma_{j+1}$, so $p$ is not an abnormal root in $\gamma_{j+1}$. Hence, $\gamma_{j+1}(q)$.status $=E B$ (by Observation 1) and, consequently, $\gamma_{j}(q)$.status $\in\{C, E B\}$.

- If $\gamma_{j}(q)$.status $=E B$, then $p$ does not execute any action in the step $\gamma_{j} \mapsto \gamma_{j+1}$ (otherwise, $\gamma_{j+1}(p)$.status $\neq C$ or $\gamma_{j+1}(p)$.par $\left.\neq q\right)$. Hence, $\gamma_{j}(p)$.status $=\gamma_{j+1}(p)$.status $=C$. By hypothesis, "p.status $=C \wedge|K \operatorname{Path}(p)| \leq d+1$ " is false in $\gamma_{j}$, so we have $|K \operatorname{Path}(p)|>d+1$ in $\gamma_{j}$. Now, $\gamma_{j}(p)$.status $=C$ and $\gamma_{j}(q)$.status $=E B$, so $S$-Trace $(K \operatorname{Path}(p))=E B^{+} C$ in $\gamma_{j}$ (Observation 1) and $p$ is the only process in its KPath that can execute an action in $\gamma_{j} \mapsto \gamma_{j+1}$. Hence, for every $q$ such that $q \in \operatorname{KPath}(p)$ in $\gamma_{j}$, we have $q \in \operatorname{KPath}(p)$ in $\gamma_{j+1}$, and consequently $|K \operatorname{Path}(p)|>d+1$ in $\gamma_{j+1}$. So p.status $=C \wedge|K \operatorname{Path}(p)| \leq d+1$ is false in $\gamma_{j+1}$, a contradiction. 
- If $\gamma_{j}(q)$.status $=C$, then $q$ is in an alive abnormal tree in $\gamma_{j}$ (indeed, $q$ executes EB-action in $\gamma_{j} \mapsto \gamma_{j+1}$, and so Lemma 8 applies). As $q$ is alive in $\gamma_{j}$, we have $|K \operatorname{Path}(q)|>d$ in $\gamma_{j}$ by induction hypothesis. Moreover, $q$ is not an abnormal root (there is no more alive abnormal root after the first round, see the base case). Hence, the status of its parent in $\gamma_{j}$ is $E B$. Now, $|K \operatorname{Path}(q)|>d$ and $S$-Trace $(K \operatorname{Path}(q))=E B^{+} C$ in $\gamma_{j}$ (Observation 1$)$. So, $q$ is the only one in its KPath that executes an action in $\gamma_{j} \mapsto \gamma_{j+1}$ and this action is EB-action, which maintains the KinshipOk relation. Hence, $|K \operatorname{Path}(q)|>d$ in $\gamma_{j+1}$ and consequently, $|K \operatorname{Path}(p)|>d+1$ in $\gamma_{j+1}$, a contradiction.

Hence, for every process $p$, if $($ p.status $=C \wedge|K \operatorname{Path}(p)| \leq d+1)$ is false in some configuration $\gamma_{i}$ with $i \geq R_{d}$, then (p.status $\left.=C \wedge|K \operatorname{Path}(p)| \leq d+1\right)$ remains false forever.

Now, EB-action is continuously enabled $\forall p$ such that $p$ is alive $|\operatorname{KPath}(p)|=d+1$ in $\gamma_{R_{d}}$ (by induction hypothesis and Lemma 18). So, $p$ becomes dead during the round and, $\forall j \geq R_{d+1}, \gamma_{j}$ contains no alive process $p$ such that $|K \operatorname{Path}(p)| \leq d+1$.

$n \geq \max \{|K \operatorname{Path}(p)|, \forall p \in V\}$. Hence, any process in an abnormal tree becomes dead in at most $n$ rounds, and we are done.

Lemma 19. If EF-action is enabled at a process $p$, it remains enabled until $p$ executes EF-action.

Proof. Assume by contradiction EF-action is enabled at a process $p$ in configuration $\gamma$ and is not enabled in the next configuration $\gamma^{\prime}$, but $p$ did not execute EF-action during the step $\gamma \mapsto \gamma^{\prime}$. Notice that $p$ does not execute any action during this step, as guards are mutually exclusive. As EFeedback $(p)$ holds in $\gamma, \gamma(p)$.status $=\gamma^{\prime}(p)$.status $=E B$. As EFeedback $(p)$ does not hold in $\gamma^{\prime}$ and no process can execute $J$-action and choose a process of status $E B$ as parent, $\exists q \in$ RealChildren $_{p}$ such that $\gamma(q)$.status $=E F$ and $\gamma^{\prime}(q)$.status $\neq E F$. Now, because $\gamma(q)$.status $=E F, q$ can only execute $R$-action. However, as $q \in$ RealChildren $_{p}$, KinshipOk $(q, p)$ holds in $\gamma$ and then $q$ is not a root. So, $q$ cannot execute any action and change its status during $\gamma \mapsto \gamma^{\prime}$, a contradiction.

Theorem 6. Let $\gamma$ be a configuration containing abnormal trees and where all abnormal trees are dead. In at most $n$ rounds from $\gamma$, the system reaches a configuration where the status of all abnormal roots is $E F$.

Proof. Consider any execution $e=\gamma_{0}, \ldots$ starting from a configuration that contains abnormal trees and where all abnormal trees are dead. $\forall i>0$, we denote by $\gamma_{R_{i}}$ the last configuration of the $i$ th round and so the first configuration of the $i+1$ th round. Moreover, let $\gamma_{R_{0}}$ be the initial configuration.

Claim 1: $\forall p \in V, \forall i \geq R_{0}$, if $\gamma_{i}(p)$.status $\neq E B$, then $\forall j \geq i, \gamma_{j}(p)$.status $\neq E B$.

Assume by contradiction that $\gamma_{j}(p)$.status $\neq E B$ and $\gamma_{j+1}(p)$.status $=E B$, with $\gamma_{j} \mapsto \gamma_{j+1}$. Then, p.status $=C$ in $\gamma_{j}$ and EB-action is enabled at $p$ in $\gamma_{j}$. So, $p$ is in an alive abnormal tree in $\gamma_{j}$ (Lemma 8), a contradiction to Lemma 3.

In any configuration $\gamma$, we denote by MaxLengthKPath $(p)=\max \{|K \operatorname{Path}(q)|, q \in V \wedge p \in$ $K \operatorname{Path}(q)\}$. Again in $\gamma$, we define $L(p)=\operatorname{MaxLengthKPath}(p)-|K \operatorname{Path}(p)|$ and $E B L(p, k) \equiv$ p.status $=E B \wedge L(p)=k$.

Claim 2: $\forall i \geq R_{0}$, if $E B L\left(p, k_{i}\right)$ holds in $\gamma_{i}$, then $\forall j \geq i, \forall k_{j}<k_{i}, \neg E B L\left(p, k_{j}\right)$ holds in $\gamma_{j}$.

If $j=i, E B L\left(p, k_{j}\right)$ is false for $k_{j}<k_{i}$ because $L(p)$ cannot have two different values in a same configuration. Assume now $j>i$. The case $k_{i}=0$ is direct. Assume $k_{i}>0$. Assume by contradiction that $E B L\left(p, k_{i}\right)$ holds in $\gamma_{i}$ and $E B L\left(p, k_{j}\right)$ holds in $\gamma_{j}$ with $j>i$ and $k_{j}<k_{i}$. So, $\gamma_{i}(p)$.status $=\gamma_{j}(p)$.status $=E B$ and there are two cases:

- p.status $=E B$ in all the configurations between $\gamma_{i}$ and $\gamma_{j}$. Consider the step $\gamma_{i} \mapsto \gamma_{i+1}$. Let $q$ be any process such that $p \in K \operatorname{Path}(q)$ in $\gamma_{i}$. So, $K \operatorname{Path}(q)=q_{0} \ldots q_{i}=p \ldots q_{k}=q$ and $S$-Trace $(K \operatorname{Path}(q))=E B^{+} E F^{*}$ in $\gamma_{i}$. There is a unique process in KPath $(q)$ that can execute an action in $\gamma_{i} \mapsto \gamma_{i+1}$ (the only one of status $E B$ with children of status $E F$ ). If it executes an action, it is EF-action which maintains KinshipOk relation. Hence, $\forall q^{\prime} \in$ 
$K \operatorname{Path}(q)$ in $\gamma_{i}, q^{\prime} \in K \operatorname{Path}(q)$ in $\gamma_{i+1}$. We can apply this latter property to every process $r$ such that $p \in K \operatorname{Path}(r)$ and $|K \operatorname{Path}(r)|=\operatorname{MaxLengthKPath}(p)$ in $\gamma_{i}: p \in K \operatorname{Path}(r)$ in $\gamma_{i+1}$ and the value of $|K \operatorname{Path}(r)|$ in $\gamma_{i+1}$ is greater than or equal to the value of $|K \operatorname{Path}(r)|$ in $\gamma_{i}$. So, $E B L\left(p, k_{i+1}\right)$ holds with $k_{i+1} \geq k_{i}$. Applying the same argument on step $\gamma_{i+1} \mapsto \gamma_{i+2}$, etc., until step $\gamma_{j-1} \mapsto \gamma_{j}$, we obtain that $E B L\left(p, k_{j}\right)$ is true in $\gamma_{j}$ with $k_{j} \geq k_{i}$, a contradiction.

- There is a configuration between $\gamma_{i}$ and $\gamma_{j}$ where p.status $\neq E B$. So, $\exists x$ such that $i<x<j$, $\gamma_{x}(p)$. status $\neq E B$ and $\gamma_{x+1}(p)$.status $=E B$. This contradicts Claim 1 .

We show by induction that $\forall i \geq R_{d}$ with $d \geq 1, \forall p \in V, \forall k \leq d-1, E B L(p, k)$ is false in $\gamma_{i}$.

Base case: There are three cases:

1. If $L(p)=0$ in $\gamma_{R_{0}}$ and $\gamma_{R_{0}}(p)$.status $=E B$, then EF-action is enabled at $p$ in $\gamma_{R_{0}}, p$ executes EF-action during the first round, by Lemma 19 and $p$ gets status EF. By Claim 1, p.status remains different from $E B$ forever and $E B L(p, 0)$ is false in $\gamma_{i}, \forall i \geq R_{1}$.

2. If $\gamma_{R_{0}}(p)$.status $\neq E B$, p.status $\neq E B$ forever (Claim 1) and then $E B L(p, 0)$ is false forever.

3. If $\operatorname{EBL}(p, k)$ holds in $\gamma_{R_{0}}$ with $k>0, E B L(p, 0)$ is false forever (Claim 2).

Induction hypothesis: $\forall i \geq R_{d}$ with $d \geq 1, \forall p \in V, \forall k \leq d-1, E B L(p, k)$ is false in $\gamma_{i}$.

Induction step: There are four cases:

1. If $L(p)=d$ and $\gamma_{R_{d}}(p)$.status $=E B, \forall q \in$ RealChildren $n_{p}$ in $\gamma_{R_{d}}, L(q)<d$ by definition and $\gamma_{R_{d}(q)}$.status $\neq E B$ by induction hypothesis. Now, the trees are dead, so $\gamma_{R_{d}}(q)$.status $=E F$. Hence, EF-action is enabled at $p$ in $\gamma_{R_{d}}, p$ executes $E F$-action during the round (Lemma 19) and gets status $E F$. By Claim 1, p.status $\neq E B$ forever so $E B L(p, d)$ is false at the end of the $d+1$ th round and remains false forever.

2. If $L(p)=d$ and $\gamma_{R_{d}}(p)$.status $\neq E B$, then p.status $\neq E B$ forever (Claim 1). So, $E B L(p, d)$ is false forever.

3. If $L(p)<d$, by induction hypothesis $\gamma_{R_{d}}(p)$.status $\neq E B$ and we conclude as in case 2 .

4. If $E B L(p, k)$ holds in $\gamma_{R_{d}}$ with $k>d, E B L(p, i)$ is false forever $\forall i \leq d$ (Claim 2).

With $d=n$, we have $\forall i \geq R_{n}, \forall p \in V, \forall k \leq n-1, E B L(p, k)$ is false in $\gamma_{i}$ : hence, in at most $n$ rounds, there is no more process of status $E B$ in abnormal trees, those ones being dead. So, all processes (and in particular the abnormal roots) in abnormal trees have status $E F$.

Lemma 20. If all abnormal trees are dead and $R$-action is enabled at a process $p$, then $R$-action remains enabled at $p$ until $p$ executes it.

Proof. Let $\gamma$ be a configuration, where all abnormal trees are dead. Assume, by contradiction, that $R$-action is enabled at a process $p$ in a configuration $\gamma$ and is not enabled in the next configuration $\gamma^{\prime}$, but $p$ did not execute $R$-action during the step $\gamma \mapsto \gamma^{\prime}$. Notice that $p$ does not execute any action during this step, as guards are mutually exclusive.

As $R$-action is enabled in $\gamma$ and $p$ does not execute an action during the step, $\gamma(p)$.status = $\gamma^{\prime}(p)$.status $=E F$.

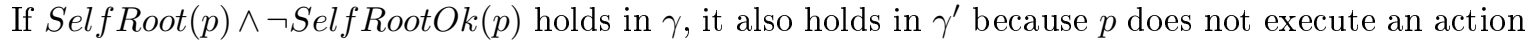
between $\gamma$ and $\gamma^{\prime}$ and these predicates only depends on the local state of $p$.

Otherwise $\neg \operatorname{SelfRoot}(p) \wedge \neg \operatorname{KinshipOk}(p$, p.par $)$ holds in $\gamma$. Let $q=p$.par. If $q$ does not execute an action between $\gamma$ and $\gamma^{\prime}, p$ is still an abnormal root. Otherwise, three cases are possible:

- $\neg \operatorname{GoodIdR}(p, q)$ holds in $\gamma$. First, if $\gamma(p)$.idR $<\gamma(q)$.idR. If $q$ executes EB-action or EF-action during the step, the $i d R$ of $q$ does not change, so $\gamma^{\prime}(p) . i d R<\gamma^{\prime}(q)$.idR, and $A b \operatorname{Root}(p)$ holds in $\gamma^{\prime}$. Otherwise $q$ executes $R$-action or J-action. Then $\gamma^{\prime}(q)$.status $=C$, so $\neg$ GoodStatus $(p, q)$ and $A b \operatorname{Root}(p)$ holds in $\gamma^{\prime}$. If $\gamma(p) . i d R \geq p$, the $i d R$ is not modified during the step, so $\gamma^{\prime}(p) . i d R=$ $\gamma(p) . i d R \geq p$ and $\operatorname{AbRoot}(p)$ holds in $\gamma^{\prime}$. 
- $\neg \operatorname{GoodLevel}(p, q)$ holds in $\gamma$. Then $\gamma(p) . i d R=\gamma(q)$.idR but $\gamma(p)$.level $\neq \gamma(q)$.level +1 . If $q$ executes EB-action or EF-action, its $i d R$ and its level do not change, so $\gamma^{\prime}(p) . i d R=\gamma^{\prime}(q) . i d R$ and $\gamma^{\prime}(p)$.level $\neq \gamma^{\prime}(q)$.level +1 , so AbRoot $(p)$ holds in $\gamma^{\prime}$. Otherwise, $q$ executes $R$-action or $J$-action. Then $\gamma^{\prime}(q)$.status $=C$, so $\neg \operatorname{GoodStatus}(p, q)$ and $A b R o o t(p)$ holds in $\gamma^{\prime}$.

- $\neg \operatorname{GoodStatus}(p, q)$ holds in $\gamma$. Then $\gamma(q)$.status $=C$, and $q$ can only execute EB-action or J-action between $\gamma$ and $\gamma^{\prime}$. If $q$ executes EB-action then EBroadcast $(q)$ holds in $\gamma$, so $q$ is in an abnormal tree (Lemma 8). But, by hypothesis, all abnormal trees are dead in $\gamma$, so $\gamma(q)$.status $\neq C$, a contradiction. If $q$ executes $J$-action then $\gamma^{\prime}(q)$ status $=C$, so $\neg \operatorname{GoodStatus}(p, q)$ and $\operatorname{AbRoot}(p)$ holds in $\gamma^{\prime}$.

Thus, $\gamma^{\prime}(p)$.status $=E F$ and $\operatorname{AbRoot}(p)$ holds in $\gamma^{\prime}$ and, consequently, Allowed $(p)$ is false in $\gamma^{\prime}$. So $\exists q \in \mathcal{N}_{p}$ such that $q \in$ Children $_{p} \wedge \neg \operatorname{KinshipOk}(q, p)$ holds in $\gamma^{\prime}$ but $\gamma^{\prime}(q)$.status $=C$. Two cases are possible:

- If $q \notin$ Children $_{p}$ in $\gamma$, then $q$ executes $J$-action during the step $\gamma \mapsto \gamma^{\prime}$ and $\operatorname{Min}_{q}=p$. But $\gamma(p)$. status $=E F$, a contradiction.

- Otherwise $q \in$ Children $_{p}$ in $\gamma$ and $\gamma(q)$.status $\neq C$. $q$ executes either EF-action and $\gamma^{\prime}(q)$.status $=$ $E F$, or $R$-action and $\gamma^{\prime}(q)$.par $\neq p$, so $q \notin$ Children $_{p}$ in $\gamma^{\prime}$, a contradiction.

Definition 13 (Abnormal process). A process $p$ is called abnormal process if and only if $p$ belongs to an abnormal tree. $p$ is said to be normal, otherwise.

As no process can join a dead abnormal tree (Remark 1) and no alive abnormal tree can be created (Lemma 3), we have the following remark:

Remark 6. In a configuration where every abnormal tree is dead, the number of abnormal processes can only decrease.

Theorem 7. Starting from a configuration where every abnormal tree is dead and the status of their roots is $E F$, there is no more abnormal processes in at most $n$ rounds.

Proof. Let $\gamma_{0}$ be a configuration where all abnormal trees are dead and the status of their roots is $E F$. By Observation 1, all abnormal processes have status $E F$ in $\gamma_{0}$. So, from $\gamma_{0}$, no process can be ever an abnormal process with a status different of $E F$ (such a process can only execute $R$-action, then it is a normal process forever, by Lemma 3). Then, by definition, the number of abnormal processes in $\gamma_{0}$ is at most $n$. Moreover, by Remark 6, it is sufficient to show that in any configuration $\gamma_{k}$ reachable from $\gamma_{0}$, if the number of abnormal processes is not null, then at least one of them becomes normal within the next round.

So, let assume that some process $p$ is abnormal in $\gamma_{k}$. Then, $\gamma_{k}(p)$.status $=E F$. By Observation 1 and Lemma 20, the initial extremity $r$ of $K \operatorname{Path}(p)$ is an abnormal process (of status $E F$ ) and executes $R$-action within the next round. After executing $R$-action, $r$ is normal (actually, $r$ becomes a self root), and we are done.

By definition, the root of a normal tree has the status $C$. So, by Observation 1, we have:

Remark 7. Every process has the status $C$ in a configuration containing no abnormal processes. Moreover, this configuration is clean.

Using Lemma 17 and Theorems 5 to 7, we can conclude:

Theorem 8. In at most $3 n$ rounds, the system reaches a clean configuration.

Then, using Theorems 4 and 8 we get:

Theorem 9 (Round Complexity). In at most $3 n+\mathcal{D}$ rounds, the system reaches a terminal configuration. 


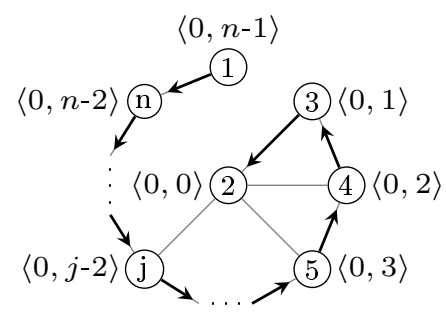

(a) The initial configuration. $\left\{p_{2}, p_{j}\right\}$ is the "last" leg $(j=k+3)$.

\section{$\langle 0, n-1\rangle$}

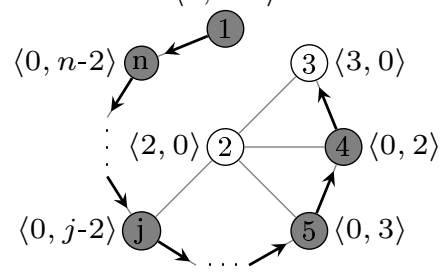

(d) $p_{2}$ and $p_{3}$ sequentially execute R-action.

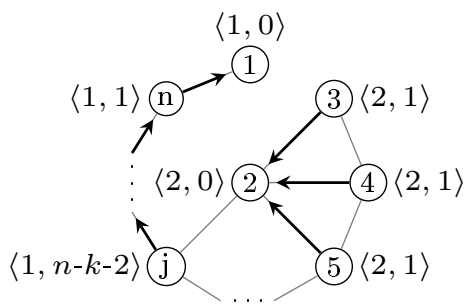

(g) In $n-k-2$ rounds, processes $n$ to $k-3$ joins $\operatorname{Tree}(1)$.

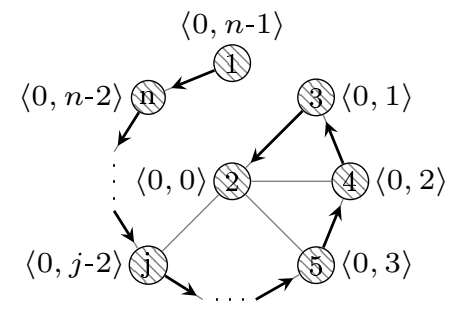

(b) In $n$ rounds, the $E B$-wave reaches $p_{1}$.

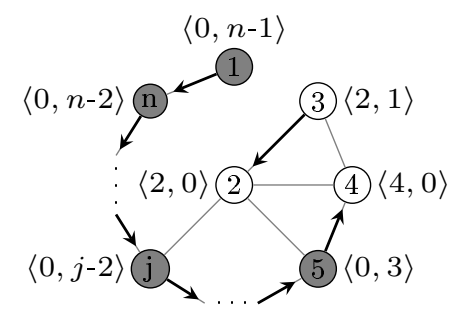

(e) $p_{3}$ executes $J$-action and $p_{4}$ simultaneously executes $R$-action.

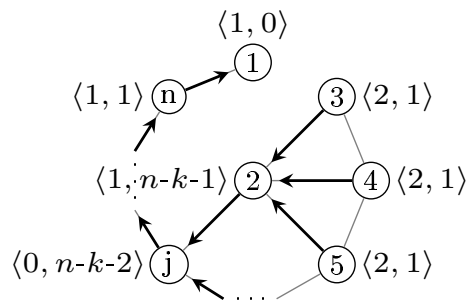

(h) Processes $p_{2}$ and $p_{k-2}$ simultaneously execute $J$-action.

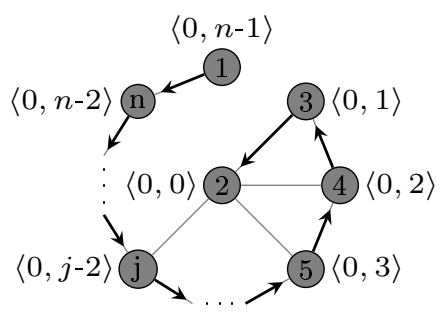

(c) In $n$ rounds, $p_{2}$ gets status $E F$.

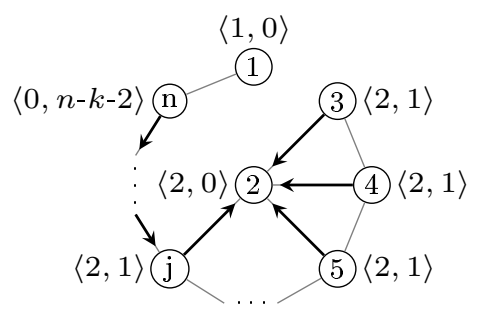

(f) In $n-3$ rounds, the cleaning is finished.

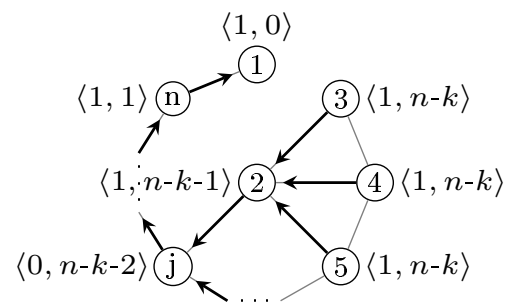

(i) In one round, the system reaches a terminal configuration where $p_{1}$ is the leader.

Figure 7: An example in $3 n+\mathcal{D}$ rounds 


\subsubsection{Worst Case Analysis of the Stabilization Time}

Lower Bound on the Worst Case Stabilization Time in Rounds. We now show that the bound proposed in Theorem 9 cannot be improved. To see this, we exhibit a construction that gives, $\forall n \geq 4$, $\forall \mathcal{D}, 2 \leq \mathcal{D} \leq n-2$, a network of $n$ processes whose diameter is $\mathcal{D}$, from which there is a possible synchronous execution that lasts exactly $3 n+\mathcal{D}$ rounds. (Recall that every synchronous execution is possible under the distributed unfair daemon.)

We consider a network $G=(V, E)$ composed of $n$ processes $V=\left\{p_{1}, \ldots, p_{n}\right\}$ such that $p_{i}$ has ID $i$, $\forall i \in[1 . . n]$. Figure 7 a shows the system in its initial configuration. In details, processes $p_{1}, p_{n}, \ldots, p_{2}$ form a chain, i.e., $\left\{p_{1}, p_{n}\right\} \in E$ and $\left\{p_{i}, p_{i-1}\right\} \in E \forall i=3 \ldots n$.

We add $k$ "legs", with $2 \leq k \leq n-2$, as follows:

If $k=n-2$, then $\left\{p_{2}, p_{1}\right\} \in E$ and $\forall i \in[4 . . n],\left\{p_{2}, p_{i}\right\} \in E$,

Otherwise $\forall i \in[4 . . k+3],\left\{p_{2}, p_{i}\right\} \in E$.

Notice that the diameter of the network is $n-k$ and can be adjusted by adding or removing some legs.

We assume the following initial configuration:

- $p_{i} . i d R=0 \forall i \in[1 . . n]$,

- $p_{1}$.level $=n-1$ and $p_{1} \cdot p a r=p_{n}$,

- $p_{2} . p a r=p_{2}$ and $p_{2} . l e v e l=0$,

- $p_{i}$.level $=i-2$ and $p_{i}$. par $=p_{i}-1, \forall i \in[3 . . n]$.

We consider a synchronous daemon, i.e. in a configuration $\gamma$, every process in Enabled $(\gamma)$ is selected by the daemon to execute an action. So, in this case, every round lasts exactly one step.

The execution is then as follows:

- $p_{2}, p_{3}, p_{4} \ldots p_{n}, p_{1}$ sequentially execute $E B$-action: $n$ rounds. (See Figure $7 \mathrm{~b}$.)

- $p_{1}, p_{n}, p_{n-1}, \ldots, p_{2}$ sequentially execute EF-action: $n$ rounds. (See Figure $7 \mathrm{c}$.)

- $p_{2}$ and $p_{3}$ sequentially execute $R$-action: 2 rounds. (See Figure $7 \mathrm{~d}$ )

- For $i=4 \ldots n$, simultaneously $p_{i}$ and $p_{i-1}$ respectively executes $R$-action and $J$-action, in particular, $p_{i-1}$ joins $\operatorname{Tree}\left(p_{2}\right): n-3$ rounds. (See Figures $7 \mathrm{e}$ and $7 \mathrm{f}$.)

- $p_{1}$ executes $R$-action and $p_{n}$ executes $J$-action simultaneously: 1 round.

- For $i=n \ldots k+3, i$ executes $J$-action to join $\operatorname{Tree}(1): n-k-2$ rounds. (See Figure 7g.)

- $p_{2}$ and $p_{k+2}$ simultaneously execute $J$-action to join Tree(1): 1 round. (See Figure 7h.)

- $p_{3}, \ldots, p_{k+1}$ simultaneously execute $J$-action and then the configuration is terminal: 1 round. (See Fig. 7i.)

Hence, the execution lasts exactly $3 n+(n-k)=3 n+\mathcal{D}$ rounds.

Lower Bound on the Worst Case Stabilization Time in Steps. We show that the bound given in Theorem 1 can be asymptotically matched, i.e., we give an example of possible execution that stabilizes in $\Omega\left(n^{3}\right)$ steps, for every $n \geq 4$.

We consider a network $G=(V, E)$ composed of $n$ processes $V=\left\{p_{1}, \ldots, p_{n}\right\}$ such that $p_{i}$ has ID $n+i, \forall i \in[1 . . n]$. Figure 8a shows the network in this initial configuration. In details, there are $2 n-3$ edges: $\left\{p_{i}, p_{i+1}\right\} \forall i=1 \ldots n-2$ and $\left\{p_{i}, p_{n}\right\} \forall i=1 \ldots n-1$. (Notice that the diameter of this network is 2.) The initial configuration is as follows:

- $p_{i} . i d R=i \forall i \in[1 . . n-1]$, and $p_{n} . i d R=2 n$.

- $p_{i}$. par $=p_{i}, p_{i}$. level $=0$ and $p_{i}$. status $=C \forall i \in[1 . . n]$. 


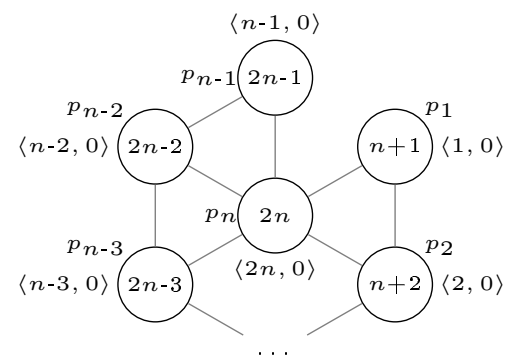

(a) The initial configuration

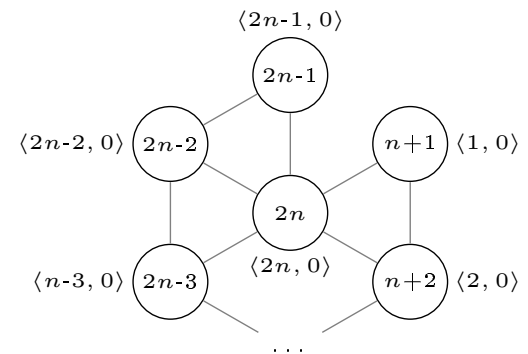

(d) In six steps, the abnormal tree rooted in $p_{n-2}$ is cleaned

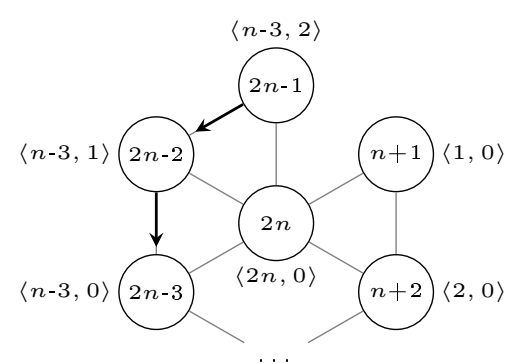

(g) $p_{n-1}$ executes $J$-action and updates its $i d R$ to $n-3$.

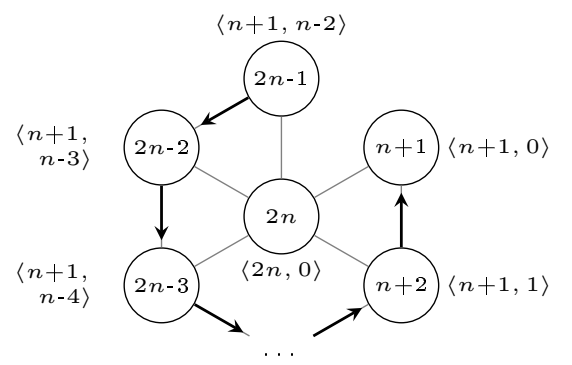

(j) In $\sum_{i=1}^{n-2} i$ steps, processes $p_{n-1}$ to $p_{2}$ elect $p_{1}$ normal
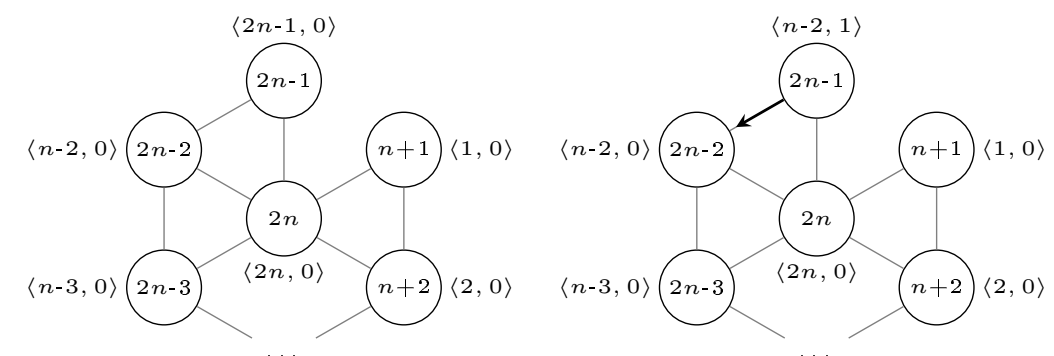

(b) In three steps, $p_{n-1}$ becomes

(c) $p_{n-1}$ executes $J$-action and joins $\operatorname{Tree}\left(p_{n-2}\right)$
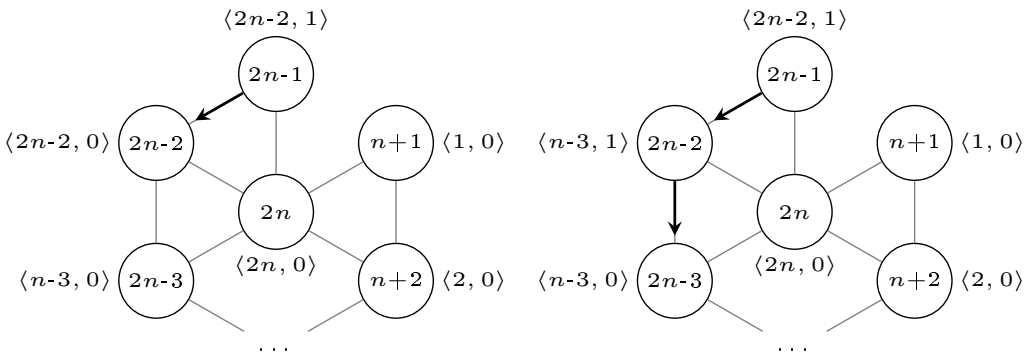

(e) $p_{n-1}$ executes $J$-action and joins the normal tree $\operatorname{Tree}\left(p_{n-2}\right)$

(f) $p_{n-2}$ executes $J$-action and joins the abnormal tree $\operatorname{Tree}\left(p_{n-3}\right)$
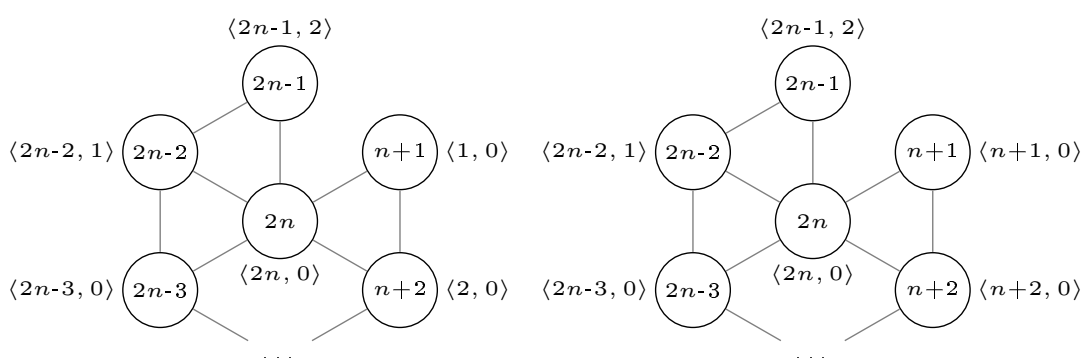

(h) In nine steps, the abnormal tree

(i) There is no more abnormal trees rooted in $p_{n-3}$ is cleaned

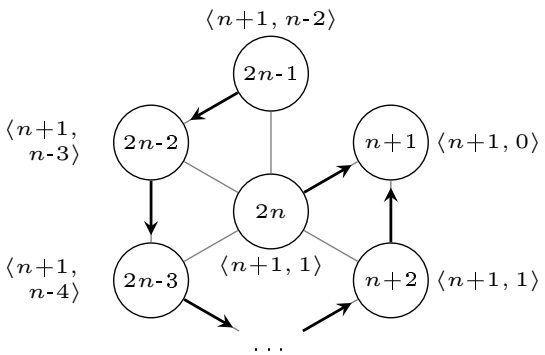

(k) In one step, the system reaches a terminal configuration where $p_{1}$ is leader.

Figure 8: An example in $\Omega\left(n^{3}\right)$ steps 
We consider the following execution:

- For $i=n-1 \ldots 1,(i--)$, we clean $\operatorname{Tree}\left(p_{i}\right)$ the following way:

1. For $j=n-2 \ldots i,(j--),{ }^{2}$

(a) For $k=j+1 \ldots n-1,(k++),{ }^{3}$

- $p_{k}$ joins $\operatorname{Tree}\left(p_{j}\right)$.

This part lasts $\sum_{k=1}^{n-1-i} k$ steps.

2. $p_{i}, p_{i+1}, \ldots, p_{n-1}$ sequentially execute $E B$-action: $n-i$ steps.

3. $p_{n-1}, p_{n-2}, \ldots, p_{i}$ sequentially execute EF-action: $n-i$ steps.

4. $p_{i}, p_{i+1}, \ldots, p_{n-1}$ sequentially execute $R$-action: $n-i$ steps.

Figures $8 \mathrm{e}$ to $8 \mathrm{~h}$ show the cleaning of $\operatorname{Tree}\left(p_{n-3}\right)$.

- After all abnormal trees have been cleaned, processes $p_{n-1}$ to $p_{2}$ join $\operatorname{Tree}\left(p_{1}\right)$ similarly as Case 1 : $\sum_{i=1}^{n-2} i$ steps (Figure $8 \mathbf{j}$ ).

- $p_{n}$ executes J-action to join $\operatorname{Tree}\left(p_{1}\right)$ : 1 step (Figure $8 \mathrm{k}$ ).

Hence, the complete execution lasts:

$$
\left(\sum_{i=1}^{n-1}\left(3(n-i)+\sum_{k=1}^{i-1} k\right)\right)+\left(\sum_{i=1}^{n-2} i\right)+1=\frac{n^{3}}{6}+\frac{5}{2} n^{2}-\frac{11}{3} n+2 \text { steps }
$$

\section{$5 \quad$ Step Complexity of Algorithm $\mathcal{D} \mathcal{L} \mathcal{V}$}

In this section, we study the step complexity of the algorithm given in [8], called here $\mathcal{D} \mathcal{L} \mathcal{V} .{ }^{4}$ Below, we show that its stabilization time is not polynomial in steps.

First, we give the code of algorithm $\mathcal{D} \mathcal{L} \mathcal{V}$ and an informal explanation of its main principles in Subsection 5.1. Then, in Subsection 5.2 we give an example of a class of network in which there is a possible execution that stabilizes in $\Omega\left(n^{4}\right)$ steps. Finally, in Subsection 5.3, we generalize the previous example to a class of network where there is a possible execution that stabilizes in $\Omega\left(n^{\alpha+1}\right)$ for any $\alpha \geq 3$.

\subsection{Overview of $\mathcal{D} \mathcal{L} \mathcal{V}$}

First, Algorithm $\mathcal{D} \mathcal{L} \mathcal{V}$ uses priorities. Each action is given with priority number. When an enabled process is selected by the daemon, it only executes its enabled action with the lowest priority number.

Algorithm $\mathcal{D} \mathcal{L} \mathcal{V}$ (refer to Algorithm 2) elects the process of minimum ID, $\ell$, and builds a minimum spanning tree rooted at $\ell$. To ensure that every process knows which one is elected, it maintains a variable leader to save its current leader. Variables parent and level are used to represent the tree. The $k e y$ of a process $p$ is the combination of its two variables p.leader and p.level. Notice that the keys are ordered by a lexical order.

When a process $p$ has a neighbor with a smaller key, $p$ executes action $J$, gets the successor key of the smaller such neighbor (BestNbrKey $(p)$ ) and chooses this latter as parent. Notice that, contrary to our algorithm, $p$ can execute action $J$ and change its parent if there is a process with the same leader but with a level smaller than p.level - 1, in order to build a minimum spanning tree.

As in $\mathcal{L} \mathcal{E}$, they define a "good relation" between a process $p$ and its parent: IsTrueChld(p). It ensures that the key of $p$ is the successor key of its parent and that its leader is smaller than its own ID. Then, a maximal set of processes linked by parent pointers and satisfying IsTrueChld relation defines a tree. The root of this tree can be a true root $(I s T r u e R o o t(p))$, i.e., the key of $p$ is its self key $(\langle p, 0\rangle)$. In this case, they said that it is a normal tree. Otherwise, the root is a false root (IsFalseRoot $(p))$, i.e., neither a true root nor a true child, and they said that it is an abnormal tree.

\footnotetext{
${ }^{2}$ Of course, when $n-2<i$, there is no iteration.

${ }^{3}$ Of course, when $j+1>n-1$, there is no iteration.

${ }^{4} \mathcal{D} \mathcal{L} \mathcal{V}$ stands for "Datta, Larmore and Vemula."
} 


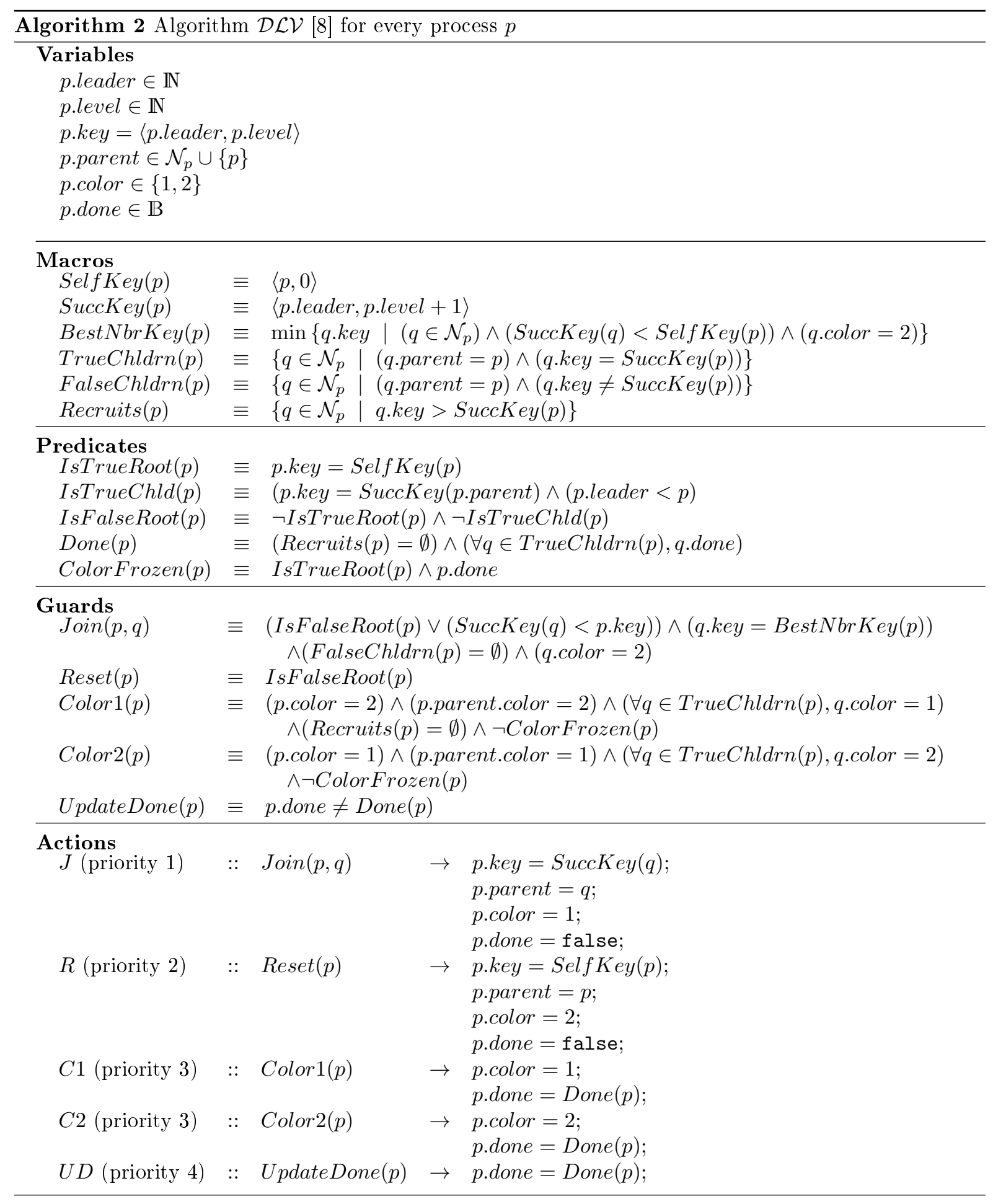


Color waves. The main difference between $\mathcal{D} \mathcal{L} \mathcal{V}$ and $\mathcal{L} \mathcal{E}$ is the way to deal with these abnormal trees. Instead of using a status and a three waves cleaning, $\mathcal{D} \mathcal{L} \mathcal{V}$ uses color waves. More precisely, each process has a variable color, either 1 or 2 . A process can only change its parent to a neighbor of color 2 and after executing action $J$, the process gets color 1 .

A process $p$ of color 2 cannot change its color to 1 when it has possible recruits $($ Recruits $(p) \neq \emptyset)$, i.e. there are some neighbors with a bigger key that may choose $p$ as parent later. Furthermore, a process can change its color, executing actions $C 1$ or $C 2$, if it has the same color than its parent (it is trivially satisfied for every true root) and if all of its true children have the other color.

To add a new level in the tree, the leaves must change their color to 2 . A first wave of actions $C 1$ is initiated by the parents of the leaves and absorbed by the root. Then, a second wave of actions $C 2$ is initiated by the leaves and also absorbed by the root. When the leaves have color 2, their neighbors can join the tree. Now, the priorities on actions prevent a false root to change its color and, so, to absorb a color wave. Moreover, every true root can always absorb a color wave.

Therefore, the colors of the processes in an abnormal tree eventually alternate, i.e., the parents and their real children do not have the same color, and no more process can join the tree: the tree is color locked. Then, the root eventually resets to a true root executing action $R$.

Once all abnormal trees have been removed, $\ell$ is a true root and regularly absorb color waves allowing then the leaves of its tree to recruit processes.

Figure 9 shows an example of execution with the cleaning of an abnormal tree.

Finally, in $O(n)$ rounds, $\ell$ is elected and a minimum spanning tree rooted at $\ell$ is built. Notice that the color waves might never end. A mechanism ensure the silence of the algorithm using the Boolean variable done and action $U D$. When a process $p$ believes that the construction of the final tree is finished (because it can no more recruits other processes) and all its true children $q$ (if any) have set their variables q.done to true, p.done is set to true. Moreover, a true root $r$ cannot change its color if $r$.done holds. We said that $r$ is color frozen. Thus, after the completion of the final tree construction, the value true is propagated bottom-up in the tree into the done variables, and in $O(\mathcal{D})$ rounds, the system reaches a terminal configuration.

\subsection{Example in $\Omega\left(n^{4}\right)$ steps}

We consider a network made of $n=L \times \beta$ processes with $L=8$ and $\beta \geq 2: p_{(1,1)}, p_{(1,2)}, \ldots, p_{(1, \beta)}$, $p_{(2,1)}, p_{(2,2)}, \ldots, p_{(2, \beta)}, \ldots, p_{(8,1)}, p_{(8,2)}, \ldots, p_{(8, \beta)}$ such that the ID of $p_{(i, j)}$ is $(i-1) \beta+j, \forall i \in[1 \ldots 8], \forall j \in$ $[1 \ldots \beta]$. Notice that 0 is a fake ID smaller than every ID in the network.

Figure 10 shows the structure of the network and the initial configuration. In details, the processes form $\beta$ columns: $\forall i \in[2 \ldots 8], \forall j \in[1 \ldots \beta],\left\{p_{(i-1, j)}, p_{(i, j)}\right\} \in V$. Moreover, there are three complete bipartite subgraphs: $\forall j \in[1 \ldots \beta], \forall j^{\prime} \in[1 \ldots \beta], j^{\prime} \neq j,\left\{p_{(4, j)}, p_{\left(5, j^{\prime}\right)}\right\},\left\{p_{(6, j)}, p_{\left(7, j^{\prime}\right)}\right\}$ and $\left\{p_{(7, j)}, p_{\left(8, j^{\prime}\right)}\right\}$ are in $V$. These bipartite subgraphs split the network in four layers:

- Layer 1: line 8

- Layer 2: line 7

- Layer 3: lines 5 and 6

- Layer 4: lines 1 to 4

We choose the following initial configuration.

- For $i \in[1 \ldots 8], j \in[1 \ldots \beta], p_{(i, j)}$. leader $=0, p_{(i, j)}$.level $=i$ and $p_{(i, j)}$.done $=$ false

- For $j \in[1 \ldots \beta]$,

$$
\begin{aligned}
& -p_{(1, j)} \cdot \text { parent }=p_{(1, j)} \\
& -p_{(5, j)} \cdot \text { parent }=p_{(4,1)} \\
& -p_{(7, j)} \cdot \text { parent }=p_{(6,1)} \\
& -p_{(8, j)} \cdot \text { parent }=p_{(7,1)} \\
& - \text { For } i \in[2 \ldots 4] \cup\{6\}, p_{(i, j)} \cdot \text { parent }=p_{(i-1, j)}
\end{aligned}
$$




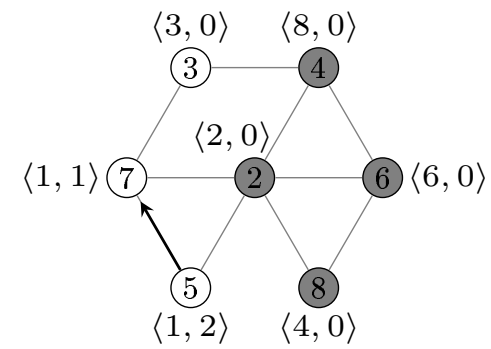

(a) Initial configuration. 1 is a fake ID.

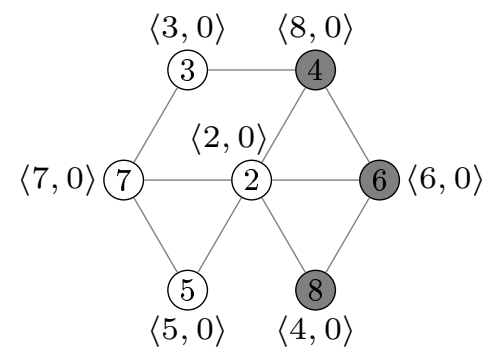

(d) 2, 3 and 5 were false roots and have executed action $R$.

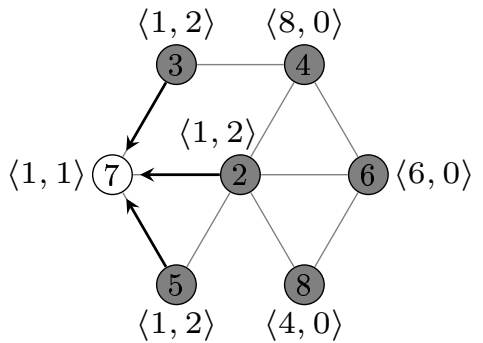

(b) 2 and 3 have executed action $J$ and chosen 7 (of color 2) as parent. 5 has changed its color to 2 executing action $C 2$.

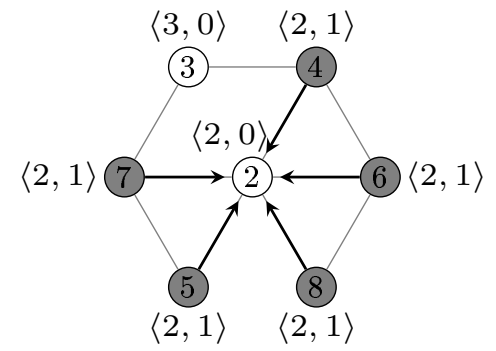

(e) 4, 5, 6, 7 and 8 have executed action $J$ and chosen 2 as parent.

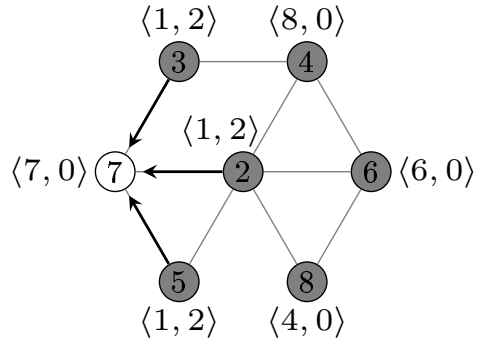

(c) The tree of 7 was color locked. Then, 7 executed action $R$.

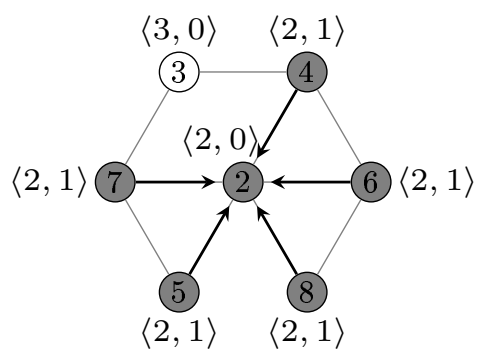

(f) 3 cannot join the tree of 2 because all its neighbors have color 1 . 2 has changed its color to 1 by executing action $C 1$.

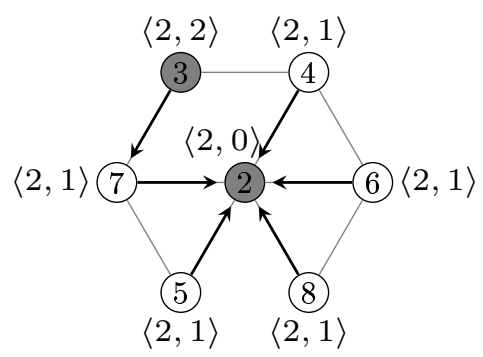

(h) Then, 3 was able to execute action $J$ and join the tree of 2 .

Figure 9: Example of execution of algorithm $\mathcal{D} \mathcal{L V}$. The ID is represented inside the node. The label next to a node shows its key. The arrows represent parent pointers. No arrow exits a node if its parent is itself. The filling represents the color: gray for 1 and white for 2 . 


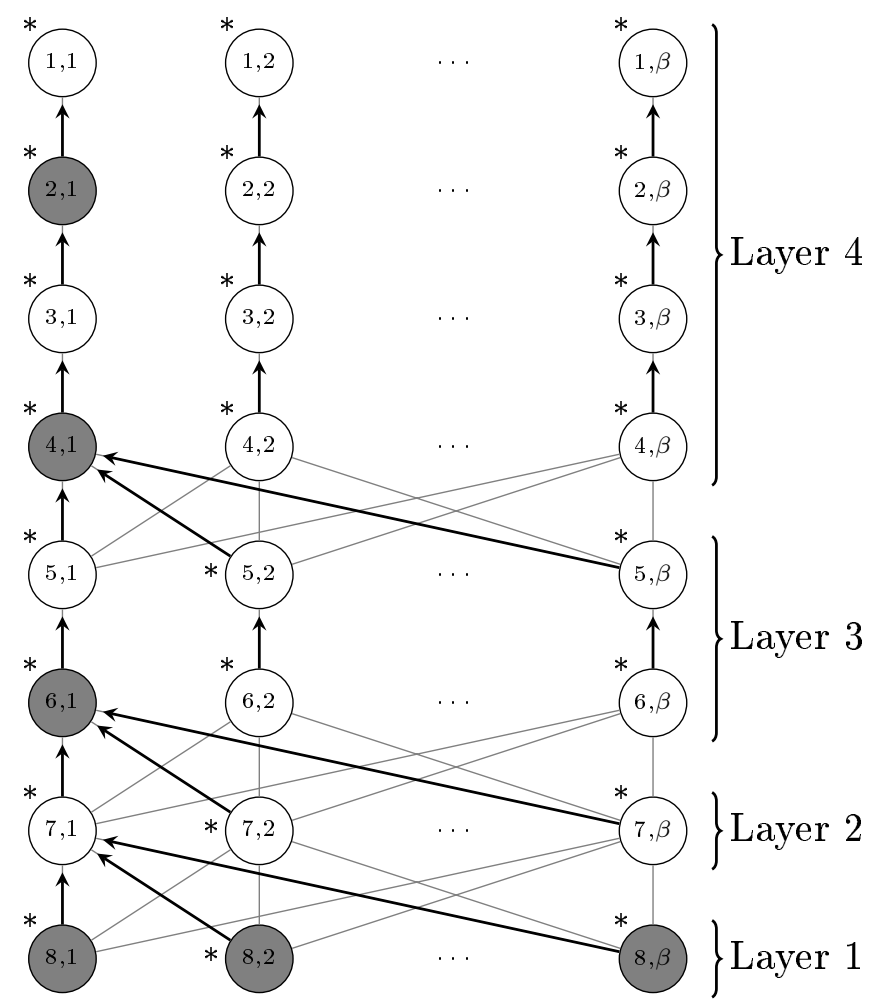

Figure 10: Initial configuration. The leader of a process is 0 if it gets a star or its own ID otherwise. level is not represented as it is always correct.

- For $i \in[1 \ldots 8], p_{(i, 1)} \cdot$ color $=(i \bmod 2)+1$

- For $j \in[2 \ldots \beta]$,

$$
\begin{aligned}
& -p_{(8, j)} \cdot \text { color }=1 \\
& - \text { For } i \in[1 \ldots 7], p_{(i, j)} \cdot \text { color }=2
\end{aligned}
$$

We consider an unfair daemon which selects the enabled processes according to function DAEMON (see Algorithm 3). In this algorithm, top $(i)$ (respectively bottom $(i)$ ) is the number of the first line (respectively last line) of layer $i$. More precisely:

$$
\begin{gathered}
\operatorname{top}(i)=L-2^{i-1}+1 \\
\operatorname{bottom}(i)= \begin{cases}\operatorname{top}(1) & \text { if } i=1 \\
\operatorname{top}(i-1)-1 & \text { if } i>1\end{cases}
\end{gathered}
$$

In $\operatorname{BuILD}($ layer, column), all the processes of lines top (layer) to 8 execute line by line action $J$. Notice that the processes of line top(layer) choose $p_{\text {(top(layer)-1,column) }}$ as parent. In $\operatorname{RESET}($ layer,column), processes $p_{(\text {top }(\text { layer }+1), \text { column })}$ to $p_{\text {(bottom(layer }+1) \text {,column) }}$ execute action $R$ (except for layer 1 where all the processes of line 8 also execute action $R$ ). Then, $\operatorname{ReSET}($ layer $-1, i)$ and $\operatorname{BuILD}($ layer $-1, i+1)$ are called for each column $i=1 \ldots \beta-1$. Finally, $\operatorname{RESET}($ layer $-1, \beta)$ is executed.

The idea is to reset a branch of the tree and then, rebuild symmetrically the tree on the next column: a process chooses as parent the neighbor of smaller key, i.e., the extreme left neighbor one line above having 0 as leader. More precisely, a first sequence of actions $R$ resets the first column and the layer 1 (Figure 11). Then, the layer 1 is rebuilt on the second column $(\operatorname{Bulld}(1,2))$ and reset again (Figure 12) etc. until the last column. Then, the tree is rebuilt since the second layer on the second column $(\operatorname{BullD}(2,2))$ and the extreme left branch is reset (Fig 13) and so on. 


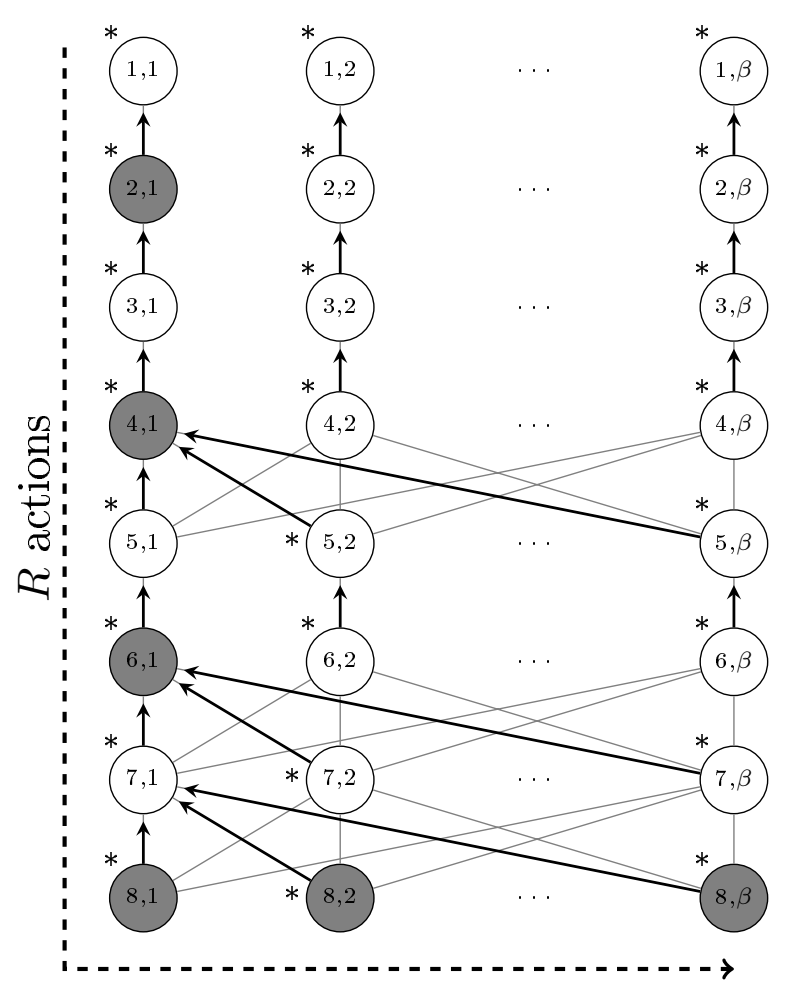

(a) Initial configuration.

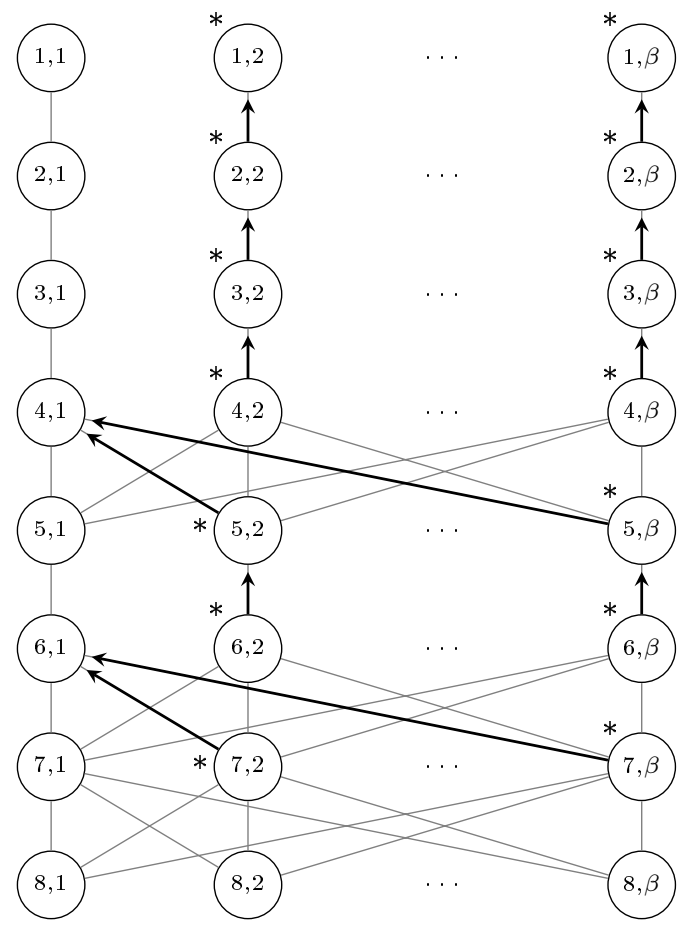

(b) Reset of the left column and the layer 1.

Figure 11: First sequence of actions $R$.

To better understand the algorithm with its numerous recursive calls, a step by step execution of function DAEMON is provided in Appendix A. The reader can follow the execution on an empty figure given with the listing.

We count how many times processes $p_{(8, .)}$ executes action $R$ :

- Each process $p_{(8, .)}$ executes once action $R$ on line 15 of Algorithm 3 in RESET(layer, column), when layer $=1$ : at least $\beta$ processes execute action $R$.

- $\operatorname{ReSeT}(3$, column $)$ is called $\beta$ times by DaEmon.

- $\operatorname{ReSet}(2$, column $)$ is called $\beta$ times by $\operatorname{ReSET}(3$, column).

- $\operatorname{ReSet}(1$, column $)$ is called $\beta$ times by $\operatorname{RESET}(2$, column).

Hence, action $R$ is executed $\beta^{4}$ times by the processes of line 8 . Now, $\beta=n / 8$. Hence, the execution lasts $\Omega\left(n^{4}\right)$ steps.

\subsection{Generalization to an example in $\Omega\left(n^{\alpha+1}\right)$ steps}

Starting from $E_{\alpha}(\alpha \geq 4)$, an example in $\Omega\left(n^{\alpha}\right)$ steps, we can build $E_{\alpha+1}$, an example in $\Omega\left(n^{\alpha+1}\right)$ steps, based on the same principle as in Subsection 5.2, by adding a layer. If $E_{\alpha}$ has $L \beta$ processes $p_{(i, j)}$ $(1 \leq i \leq L, 1 \leq j \leq \beta)$, then $E_{\alpha+1}$ has $L^{\prime}=2 L$ lines of $\beta$ processes $q_{\left(i^{\prime}, j^{\prime}\right)}\left(1 \leq i^{\prime} \leq L^{\prime}, 1 \leq j^{\prime} \leq \beta\right)$. The construction principle is as follows:

1. We increase the level and the ID of the $L \beta$ processes of $E_{\alpha}$ as follows: $\forall i \in[1 \ldots L], \forall j \in[1 \ldots \beta]$, $q_{(i+L, j)}=p_{(i, j)}$. The ID of $q_{(i+L, j)}$ becomes $(i+L-1) \beta+j$ and $q_{(i+L, j)}$.level $=i+L$. The value of variables color and done do not change. If $i \neq 1$, the parent remains the same. Otherwise, see step 3 .

2. At the top of $E_{\alpha}$, we add $L$ lines of $\beta$ processes. These new processes satisfy: 


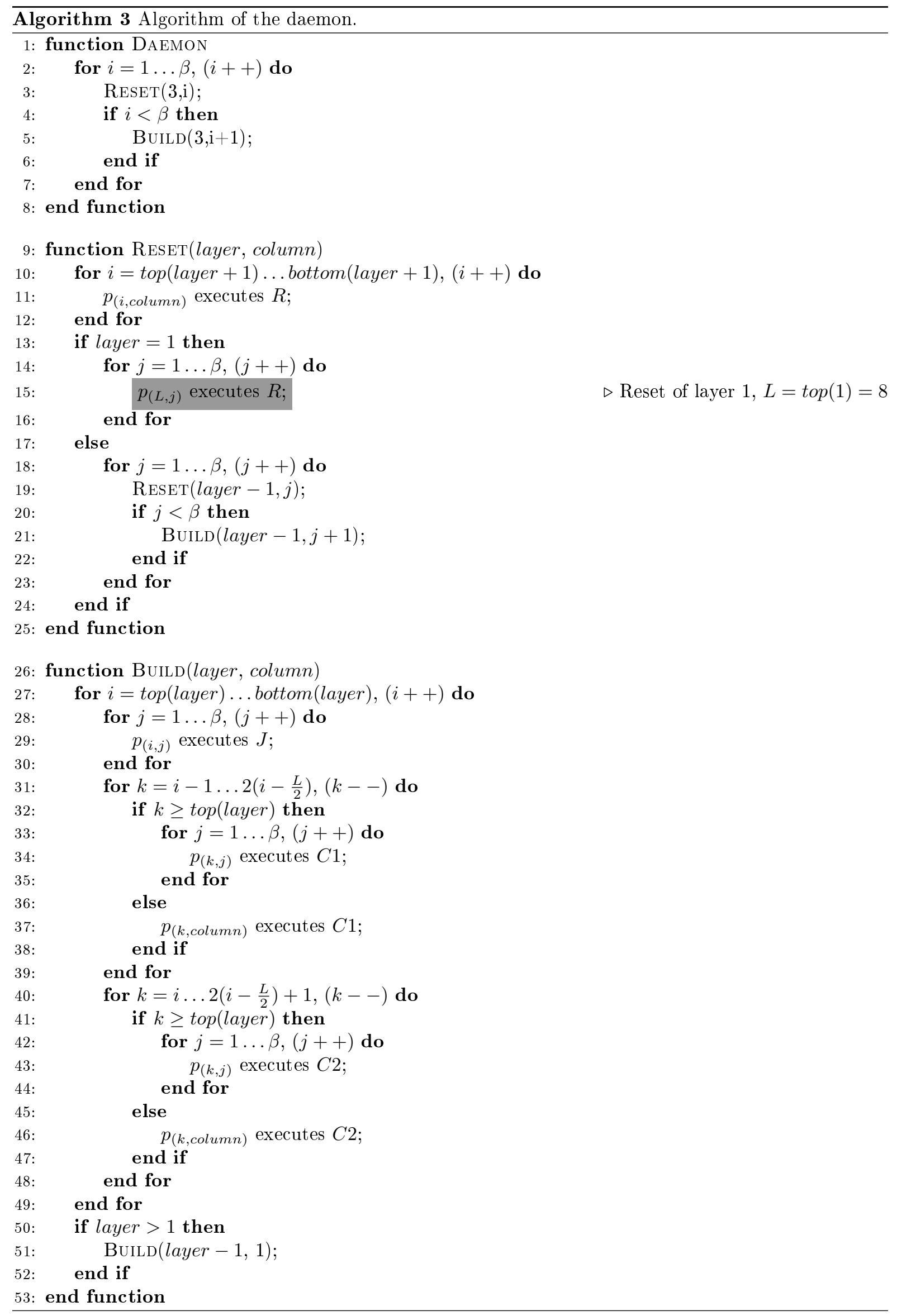




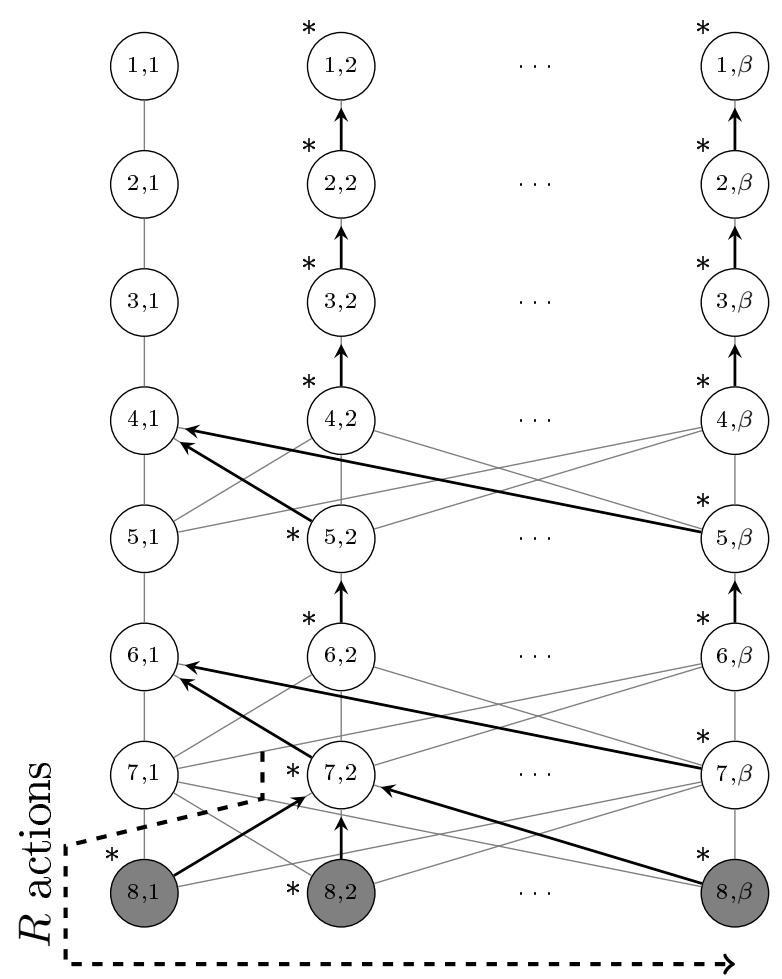

(a) $\operatorname{Build}(1,2)$

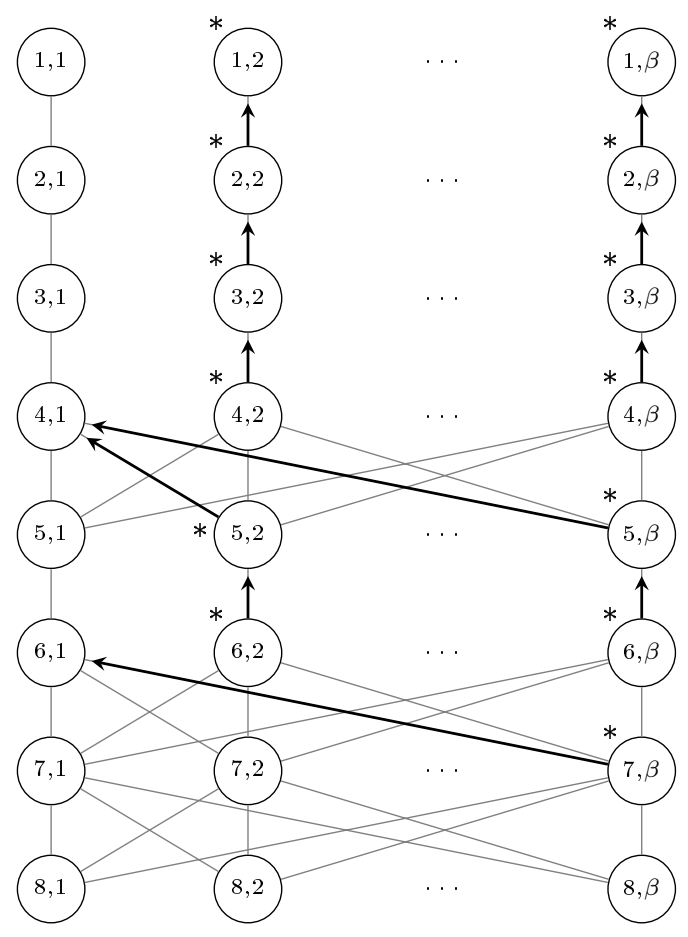

(b) Reset.

Figure 12: Reconstruction of the layer 1 on the second column and reset.

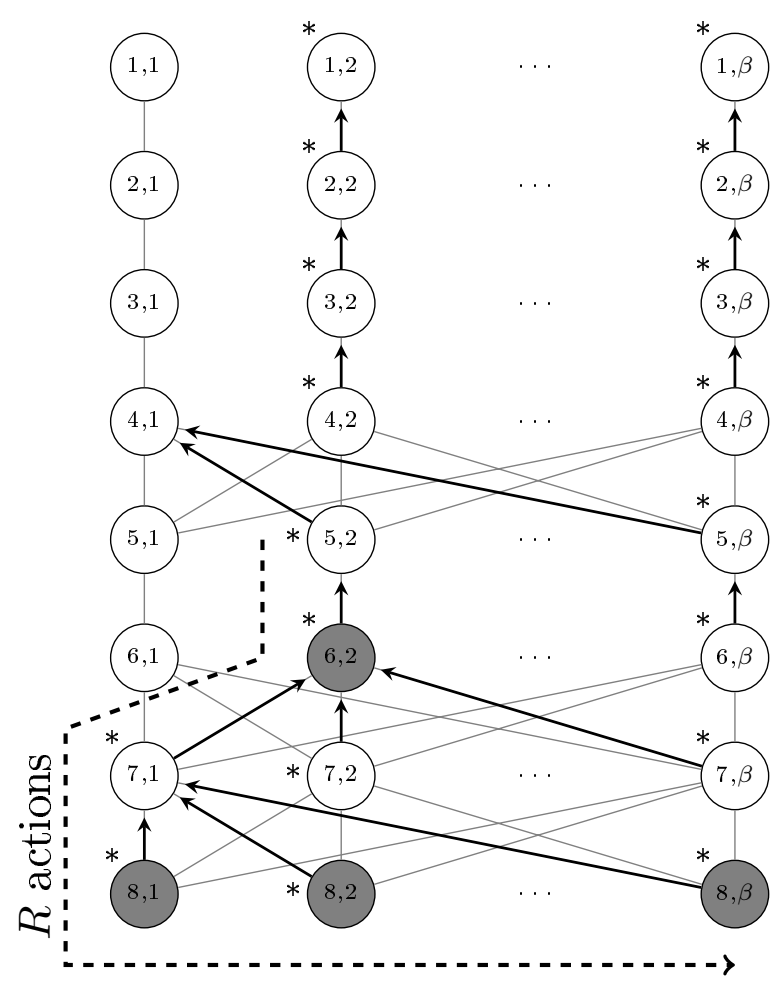

(a) $\operatorname{Build}(2,2)$

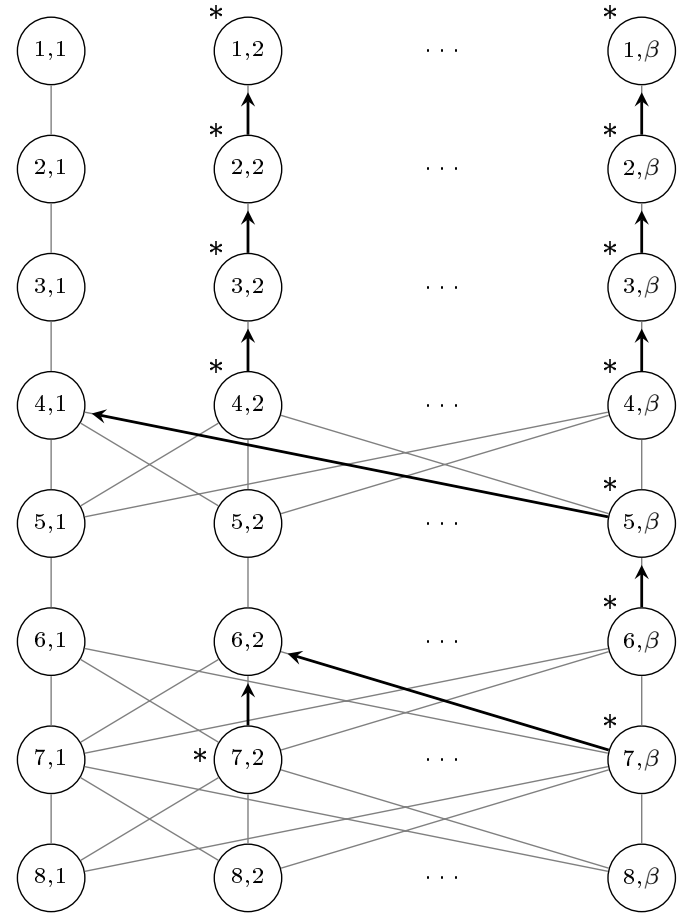

(b) Reset.

Figure 13: Reconstruction of the layer 2 on the second column and reset. 
- $\forall i \in[1 \ldots L], \forall j \in[1 \ldots \beta], q_{(i, j)} . i d=(i-1) \beta+j, q_{(i, j)}$. leader $=0, q_{(i, j)}$. level $=i$ and $q_{(i, j)}$. done $=$ false.

- $\forall i \in[2 \ldots L], \forall j \in[1 \ldots \beta],\left\{q_{(i-1, j)}, q_{(i, j)}\right\} \in V$ and $q_{(i, j)}$.parent $=q_{(i-1, j)}$.

- $\forall j \in[1 \ldots \beta], q_{(1, j)}$.parent $=q_{(1, j)}$.

- $\forall j \in[2 \ldots \beta], \forall i \in[1 \ldots L], q_{(i, j)}$. color $=2$.

- $\forall i \in[1 \ldots L], q_{(i, 1)} \cdot$ color $=(i \bmod 2)+1$.

3. The former first line of $E_{\alpha}$ becomes a new bipartite complete subgraph with the last added line:

- $\forall j \in[1 \ldots \beta], \forall j^{\prime} \in[1 \ldots \beta],\left\{q_{(L, j)}, q_{\left(L+1, j^{\prime}\right)}\right\} \in V$.

- $\forall j \in[1 \ldots \beta], q_{(L+1, j)}$.parent $=q_{(L, 1)}$.

Figure 14 shows the structure of the network for $E_{5}$ and its initial configuration.

Then, the daemon selects processes according to function DaEmon $(\alpha+1)$ (see Algorithm 4) which is the generalization of the algorithm presented in section 5.2. In $E_{\alpha}$, processes $p_{(L, .)}$ execute $\beta^{\alpha}$ times action $R$. Now, we added a new level of recursion. Then, processes $q_{\left(L^{\prime}, .\right)}$ execute $\beta^{\alpha+1}$ times action $R$. $\beta=\frac{n}{L^{\prime}}$ hence the execution lasts $\Omega\left(n^{\alpha+1}\right)$ steps.

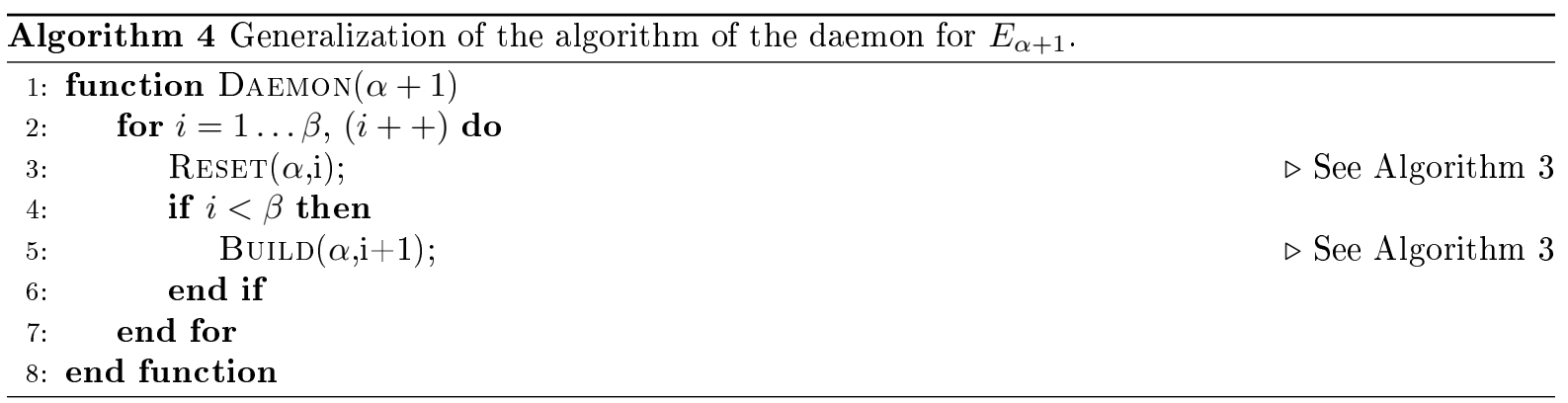

Then, for every $\alpha \geq 3$, we can build a network $E_{\alpha+1}$ such that there is a execution that lasts $\Omega\left(n^{\alpha+1}\right)$ steps. So, the stabilization time of $\mathcal{D} \mathcal{L} \mathcal{V}$ in steps is not polynomial.

\section{Conclusion}

We proposed a silent self-stabilizing leader election algorithm, called $\mathcal{L} \mathcal{E}$, for bidirectional connected identified networks of arbitrary topology. Starting from any arbitrary configuration, $\mathcal{L} \mathcal{E}$ converges to a terminal configuration, where all processes know the ID of the leader, this latter being the process of minimum ID. Moreover, as in most of the solutions from the literature, a distributed spanning tree rooted at the leader is defined in the terminal configuration.

$\mathcal{L E}$ is written in the locally shared memory model. It assumes the distributed unfair daemon, the most general scheduling hypothesis of the model. Moreover, it requires no global knowledge on the network (such as an upper bound on the diameter or the number of processes, for example). $\mathcal{L} \mathcal{E}$ is asymptotically optimal in space, as it requires $\Theta(\log n)$ bits per process, where $n$ is the size of the network. We analyzed its stabilization time both in rounds and steps. We showed that $\mathcal{L} \mathcal{E}$ stabilizes in at most $3 n+\mathcal{D}$ rounds, where $\mathcal{D}$ is the diameter of the network. We also proved that for every $n \geq 4$, for every $\mathcal{D}, 2 \leq \mathcal{D} \leq n-2$, there is a network of $n$ processes, in which a possible execution exactly lasts this complexity.

Finally, we proved that $\mathcal{L} \mathcal{E}$ achieves a stabilization time polynomial in steps. More precisely, its stabilization time is at most $\frac{n^{3}}{2}+2 n^{2}+\frac{n}{2}+1$ steps. Then, we showed for every $n \geq 4$, there exists a network of $n$ processes, in which a possible execution exactly lasts $\frac{n^{3}}{6}+\frac{5}{2} n^{2}-\frac{11}{3} n+2$ steps, establishing then that the worst case is in $\Theta\left(n^{3}\right)$.

Perspectives of this work deal with complexity issues. In [8], Datta et al showed that it is easy to implement a silent self-stabilizing leader election which works assuming an unfair daemon, uses $\Theta(\log n)$ bits per process, and stabilizes in $O(D)$ rounds (where $D$ is an upper bound on $\mathcal{D}$ ), yet if processes have knowledge of $D$. Now, it is worth investigating if it is possible to design an algorithm which works assuming an unfair daemon, uses $\Theta(\log n)$ bits per process, and stabilizes in $O(\mathcal{D})$ rounds without using any global knowledge. We believe this problem remains difficult, even adding some fairness assumption. 


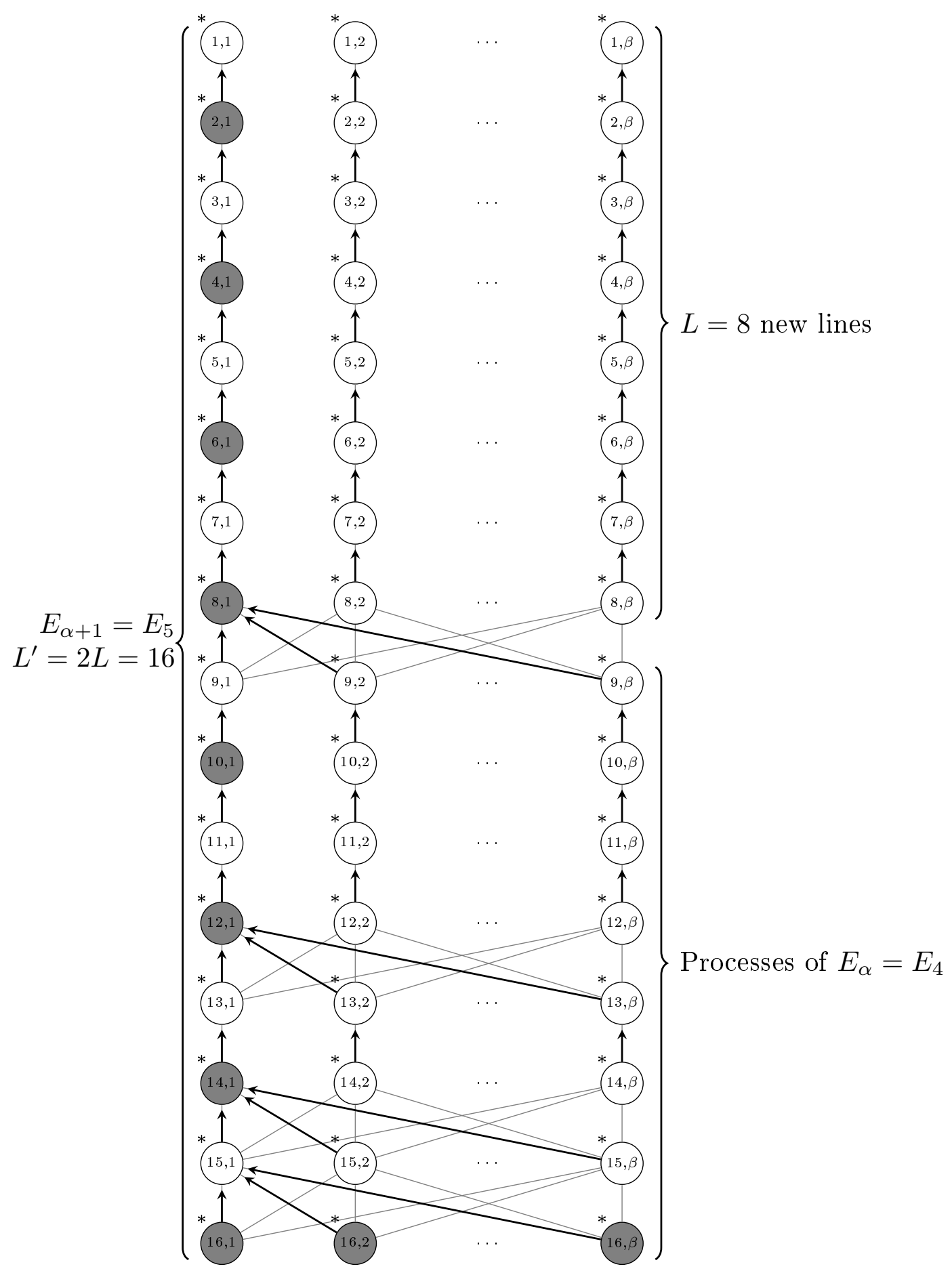

Figure 14: Initial configuration of the example in $O\left(n^{5}\right)$ steps. 


\section{References}

[1] Yehuda Afek and Anat Bremler-Barr. Self-Stabilizing Unidirectional Network Algorithms by Power Supply. Chicago J. Theor. Comput. Sci., 1998, 1998.

[2] Anish Arora and Mohamed G. Gouda. Distributed Reset. IEEE Trans. Computers, 43(9):1026-1038, 1994.

[3] Baruch Awerbuch, Shay Kutten, Yishay Mansour, Boaz Patt-Shamir, and George Varghese. Time Optimal Self-stabilizing Synchronization. In Proceedings of the Twenty-Fifth Annual ACM Symposium on Theory of Computing (STOC), pages 652-661, 1993.

[4] Janna Burman and Shay Kutten. Time Optimal Asynchronous Self-stabilizing Spanning Tree. In Distributed Computing, 21st International Symposium (DISC), pages 92-107, 2007.

[5] Ernest J. H. Chang. Echo Algorithms: Depth Parallel Operations on General Graphs. IEEE Trans. Software Eng., 8(4):391-401, 1982.

[6] Ajoy Kumar Datta, Lawrence L. Larmore, and Hema Piniganti. Self-stabilizing Leader Election in Dynamic Networks. In Stabilization, Safety, and Security of Distributed Systems - 12th International Symposium (SSS), pages 35-49, 2010.

[7] Ajoy Kumar Datta, Lawrence L. Larmore, and Priyanka Vemula. An O(n)-time Self-stabilizing Leader Election Algorithm. J. Parallel Distrib. Comput., 71(11):1532-1544, 2011.

[8] Ajoy Kumar Datta, Lawrence L. Larmore, and Priyanka Vemula. Self-stabilizing Leader Election in Optimal Space under an Arbitrary Scheduler. Theor. Comput. Sci., 412(40):5541-5561, 2011.

[9] Edsger W. Dijkstra. Self-stabilizing Systems in Spite of Distributed Control. Commun. ACM, 17(11):643-644, 1974.

[10] Shlomi Dolev. Self-stabilization. MIT Press, March 2000.

[11] Shlomi Dolev, Mohamed G. Gouda, and Marco Schneider. Memory Requirements for Silent Stabilization. Acta Inf., 36(6):447-462, 1999.

[12] Shlomi Dolev and Ted Herman. Superstabilizing Protocols for Dynamic Distributed Systems. Chicago J. Theor. Comput. Sci., 1997, 1997.

[13] Alex Kravchik and Shay Kutten. Time Optimal Synchronous Self Stabilizing Spanning Tree. In DISC, pages 91-105, 2013.

[14] Adrian Segall. Distributed Network Protocols. IEEE Transactions on Information Theory, 29(1):23$34,1983$. 
Listing: Step by step execution of $\mathcal{D} \mathcal{L V}$ in $E_{4}$ with $\beta=3$ following DaEmon

$(1,1)$ executes $\mathbf{R}$

$(2,1)$ executes $R$

$(3,1)$ executes $R$

$(4,1)$ executes $\mathrm{R}$

$(5,1)$ executes $\mathrm{R}$

$(6,1)$ executes $R$

$(7,1)$ executes $R$

$(8,1),(8,2),(8,3)$ execute $\mathrm{R}$

$(8,1),(8,2),(8,3)$ execute J

$(7,2)$ executes $R$

$(8,1),(8,2),(8,3)$ execute $\mathrm{R}$

$(8,1),(8,2),(8,3)$ execute J

$(7,3)$ executes $R$

$(8,1),(8,2),(8,3)$ execute R

$(7,1),(7,2),(7,3)$ execute J

$(6,2)$ executes $\mathrm{C} 1$

$(7,1),(7,2),(7,3)$ execute C2

$(8,1),(8,2),(8,3)$ execute J

$(5,2)$ executes $\mathrm{R}$

$(6,2)$ executes $R$

$(7,1)$ executes $\mathrm{R}$

$(8,1),(8,2),(8,3)$ execute $\mathbf{R}$

$(8,1),(8,2),(8,3)$ execute J

$(7,2)$ executes $\mathrm{R}$

$(8,1),(8,2),(8,3)$ execute R

$(8,1),(8,2),(8,3)$ execute J

$(7,3)$ executes $R$

$(8,1),(8,2),(8,3)$ execute $\mathrm{R}$

$(7,1),(7,2),(7,3)$ execute J

$(6,3)$ executes $\mathrm{C} 1$

$(7,1),(7,2),(7,3)$ execute C2

$(8,1),(8,2),(8,3)$ execute J

$(5,3)$ executes $R$

$(6,3)$ executes $R$

$(7,1)$ executes $R$

$(8,1),(8,2),(8,3)$ execute $\mathrm{R}$

$(8,1),(8,2),(8,3)$ execute J

$(7,2)$ executes $R$

$(8,1),(8,2),(8,3)$ execute R

$(8,1),(8,2),(8,3)$ execute J

$(7,3)$ executes $R$

$(8,1),(8,2),(8,3)$ execute $\mathrm{R}$

$(5,1),(5,2),(5,3)$ execute J

$(4,2)$ executes $\mathrm{C} 1$

$(3,2)$ executes $\mathrm{C} 1$

$(2,2)$ executes $\mathrm{C} 1$

$(5,1),(5,2),(5,3)$ execute C2

$(4,2)$ executes C2

$(3,2)$ executes $\mathrm{C} 2$

$(6,1),(6,2),(6,3)$ execute J

$(5,1),(5,2),(5,3)$ execute $\mathrm{C} 1$

$(4,2)$ executes $\mathrm{C} 1$

$(6,1),(6,2),(6,3)$ execute C2
$(5,1),(5,2),(5,3)$ execute C2

$(7,1),(7,2),(7,3)$ execute J

$(6,1)$ executes $\mathrm{C} 1$

$(7,1),(7,2),(7,3)$ execute C2

$(8,1),(8,2),(8,3)$ execute J

$(1,2)$ executes $\mathrm{R}$

$(2,2)$ executes $\mathbf{R}$

$(3,2)$ executes $\mathrm{R}$

$(4,2)$ executes $\mathbf{R}$

$(5,1)$ executes $\mathrm{R}$

$(6,1)$ executes $\mathrm{R}$

$(7,1)$ executes $\mathrm{R}$

$(8,1),(8,2),(8,3)$ execute $R$

$(8,1),(8,2),(8,3)$ execute $\mathrm{J}$

$(7,2)$ executes $\mathrm{R}$

$(8,1),(8,2),(8,3)$ execute $R$

$(8,1),(8,2),(8,3)$ execute J

$(7,3)$ executes $R$

$(8,1),(8,2),(8,3)$ execute $\mathrm{R}$

$(7,1),(7,2),(7,3)$ execute J

$(6,2)$ executes $\mathrm{C} 1$

$(7,1),(7,2),(7,3)$ execute C2

$(8,1),(8,2),(8,3)$ execute J

$(5,2)$ executes $\mathrm{R}$

$(6,2)$ executes $\mathrm{R}$

$(7,1)$ executes $\mathrm{R}$

$(8,1),(8,2),(8,3)$ execute $R$

$(8,1),(8,2),(8,3)$ execute J

$(7,2)$ executes $R$

$(8,1),(8,2),(8,3)$ execute $R$

$(8,1),(8,2),(8,3)$ execute J

$(7,3)$ executes $R$

$(8,1),(8,2),(8,3)$ execute $R$

$(7,1),(7,2),(7,3)$ execute J

$(6,3)$ executes $\mathrm{C} 1$

$(7,1),(7,2),(7,3)$ execute $\mathrm{C} 2$

$(8,1),(8,2),(8,3)$ execute J

$(5,3)$ executes $\mathrm{R}$

$(6,3)$ executes $\mathrm{R}$

$(7,1)$ executes $R$

$(8,1),(8,2),(8,3)$ execute $\mathrm{R}$

$(8,1),(8,2),(8,3)$ execute $\mathrm{J}$

$(7,2)$ executes $R$

$(8,1),(8,2),(8,3)$ execute $\mathrm{R}$

$(8,1),(8,2),(8,3)$ execute J

$(7,3)$ executes $\mathrm{R}$

$(8,1),(8,2),(8,3)$ execute $\mathrm{R}$

$(5,1),(5,2),(5,3)$ execute J

$(4,3)$ executes $\mathrm{C} 1$

$(3,3)$ executes $\mathrm{C} 1$

$(2,3)$ executes $\mathrm{C} 1$

$(5,1),(5,2),(5,3)$ execute $\mathrm{C} 2$

$(4,3)$ executes C2
$(3,3)$ executes $\mathrm{C} 2$

$(6,1),(6,2),(6,3)$ execute J

$(5,1),(5,2),(5,3)$ execute $\mathrm{C} 1$

$(4,3)$ executes $\mathrm{C} 1$

$(6,1),(6,2),(6,3)$ execute C2

$(5,1),(5,2),(5,3)$ execute C2

$(7,1),(7,2),(7,3)$ execute J

$(6,1)$ executes $\mathrm{C} 1$

$(7,1),(7,2),(7,3)$ execute $\mathrm{C} 2$

$(8,1),(8,2),(8,3)$ execute J

$(1,3)$ executes $R$

$(2,3)$ executes $\mathrm{R}$

$(3,3)$ executes $\mathrm{R}$

$(4,3)$ executes R

$(5,1)$ executes $\mathrm{R}$

$(6,1)$ executes $R$

$(7,1)$ executes $R$

$(8,1),(8,2),(8,3)$ execute $R$

$(8,1),(8,2),(8,3)$ execute J

$(7,2)$ executes $R$

$(8,1),(8,2),(8,3)$ execute R

$(8,1),(8,2),(8,3)$ execute J

$(7,3)$ executes $R$

$(8,1),(8,2),(8,3)$ execute $\mathbf{R}$

$(7,1),(7,2),(7,3)$ execute J

$(6,2)$ executes $\mathrm{C} 1$

$(7,1),(7,2),(7,3)$ execute $C 2$

$(8,1),(8,2),(8,3)$ execute J

$(5,2)$ executes $R$

$(6,2)$ executes $\mathrm{R}$

$(7,1)$ executes $\mathrm{R}$

$(8,1),(8,2),(8,3)$ execute $R$

$(8,1),(8,2),(8,3)$ execute J

$(7,2)$ executes $\mathrm{R}$

$(8,1),(8,2),(8,3)$ execute $\mathrm{R}$

$(8,1),(8,2),(8,3)$ execute J

$(7,3)$ executes $\mathrm{R}$

$(8,1),(8,2),(8,3)$ execute $R$

$(7,1),(7,2),(7,3)$ execute J

$(6,3)$ executes $\mathrm{C} 1$

$(7,1),(7,2),(7,3)$ execute C2

$(8,1),(8,2),(8,3)$ execute J

$(5,3)$ executes $\mathrm{R}$

$(6,3)$ executes $\mathrm{R}$

$(7,1)$ executes $R$

$(8,1),(8,2),(8,3)$ execute $R$

$(8,1),(8,2),(8,3)$ execute J

$(7,2)$ executes $\mathrm{R}$

$(8,1),(8,2),(8,3)$ execute $R$

$(8,1),(8,2),(8,3)$ execute J

$(7,3)$ executes $R$

$(8,1),(8,2),(8,3)$ execute $R$ 


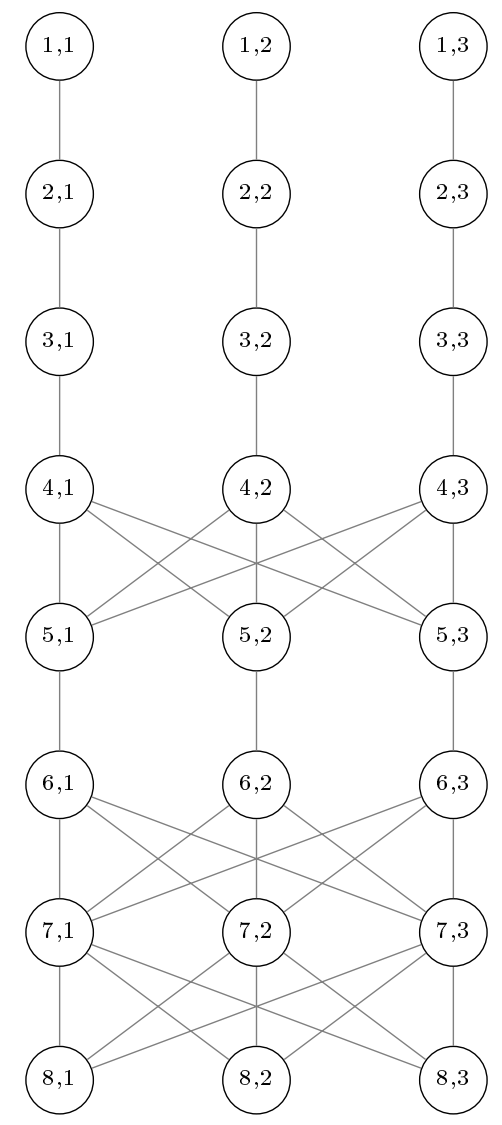

Figure 15: Empty representation of the network for $E_{4}$ with $\beta=3$. The reader can use it to follow the step by step execution. 